\title{
How Good is Jarzynski’s Equality for Computer-Aided Drug Design?
}

Kiet $\mathrm{Ho}^{1,2,+}$, Duc Toan Truong ${ }^{1,2,+}$, and Mai Suan $\mathrm{Li}^{3,{ }^{*}}$

${ }^{1}$ Institute for Computational Sciences and Technology, SBI building, Quang Trung Software

City, Tan Chanh Hiep Ward, District 12, Ho Chi Minh City, Vietnam

${ }^{2}$ Department of Theoretical Physics, Faculty of Physics and Engineering Physics, Ho Chi

Minh University of Science, Vietnam

${ }^{3}$ Institute of Physics, Polish Academy of Sciences, Al. Lotnikow 32/46, 02-668 Warsaw,

\section{Poland}

+ Contributed equally

*Email:masli@ifpan.edu.pl

\section{Supporting informartion}

Table S1 - Inhibition constant, molecular weight, chemical formula and 2D structure of the 23 studied ligands of thrombin. Their ID is given in the third column, while the second column refers to PDB ID of their complex with thrombin protein.

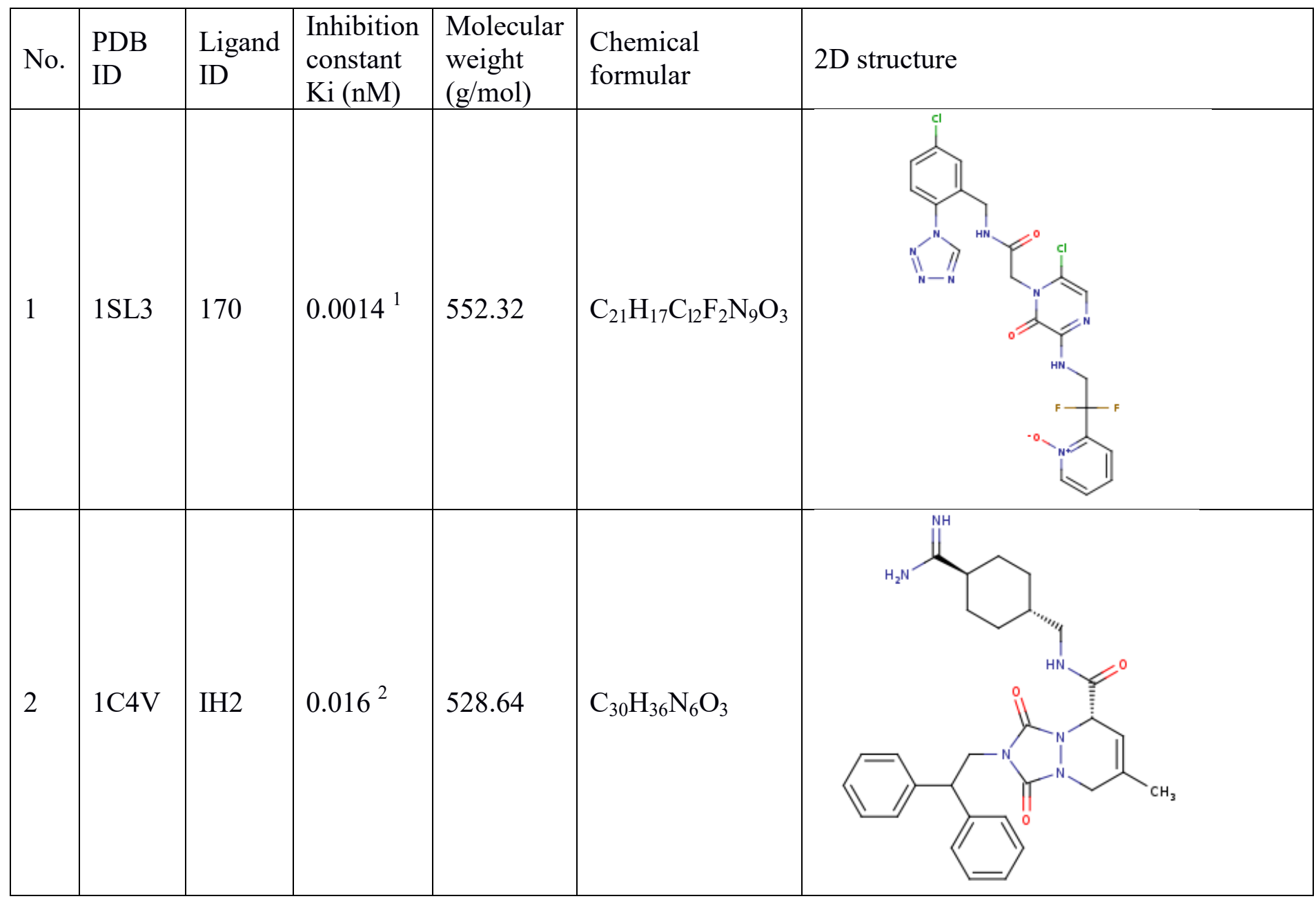




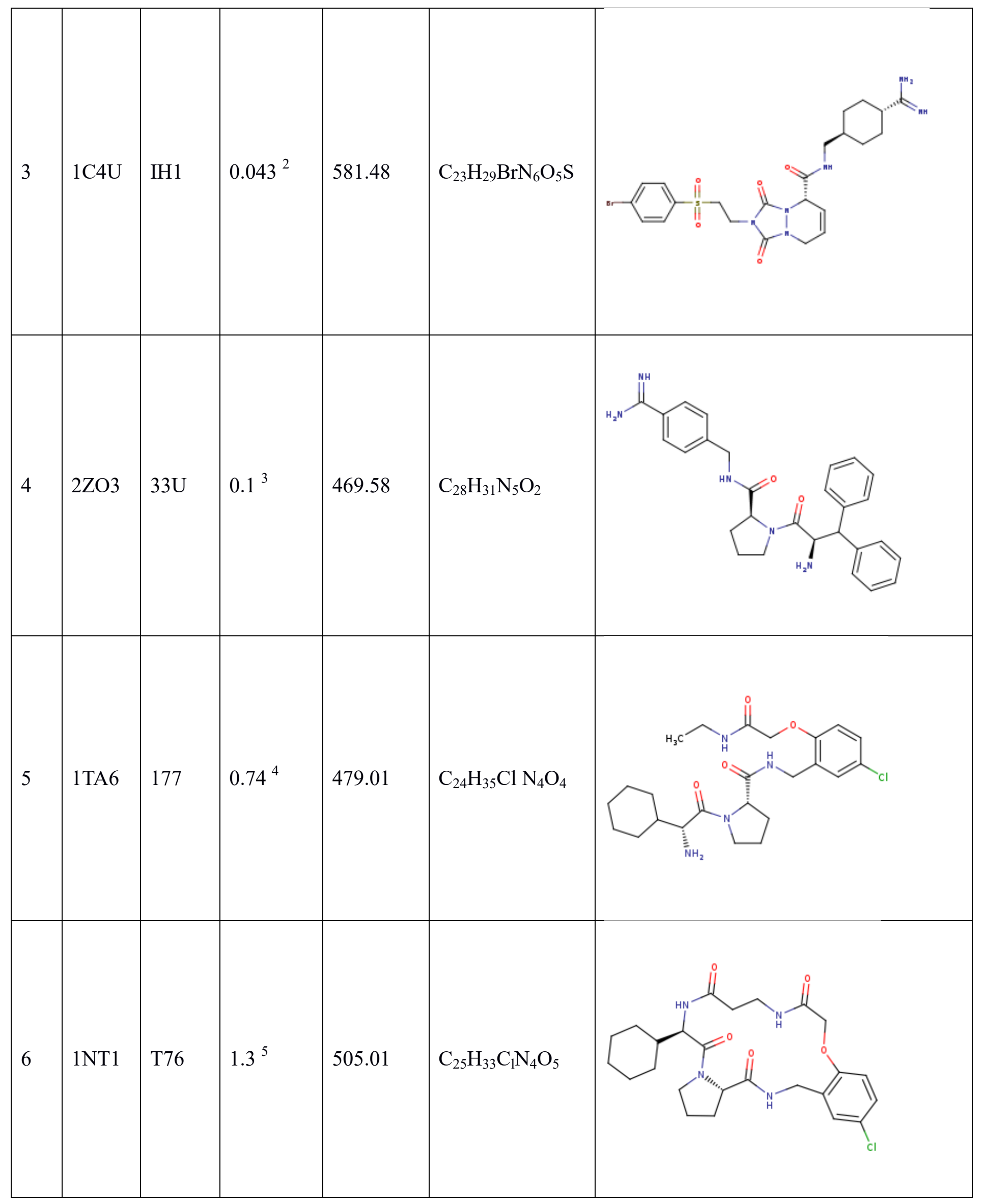




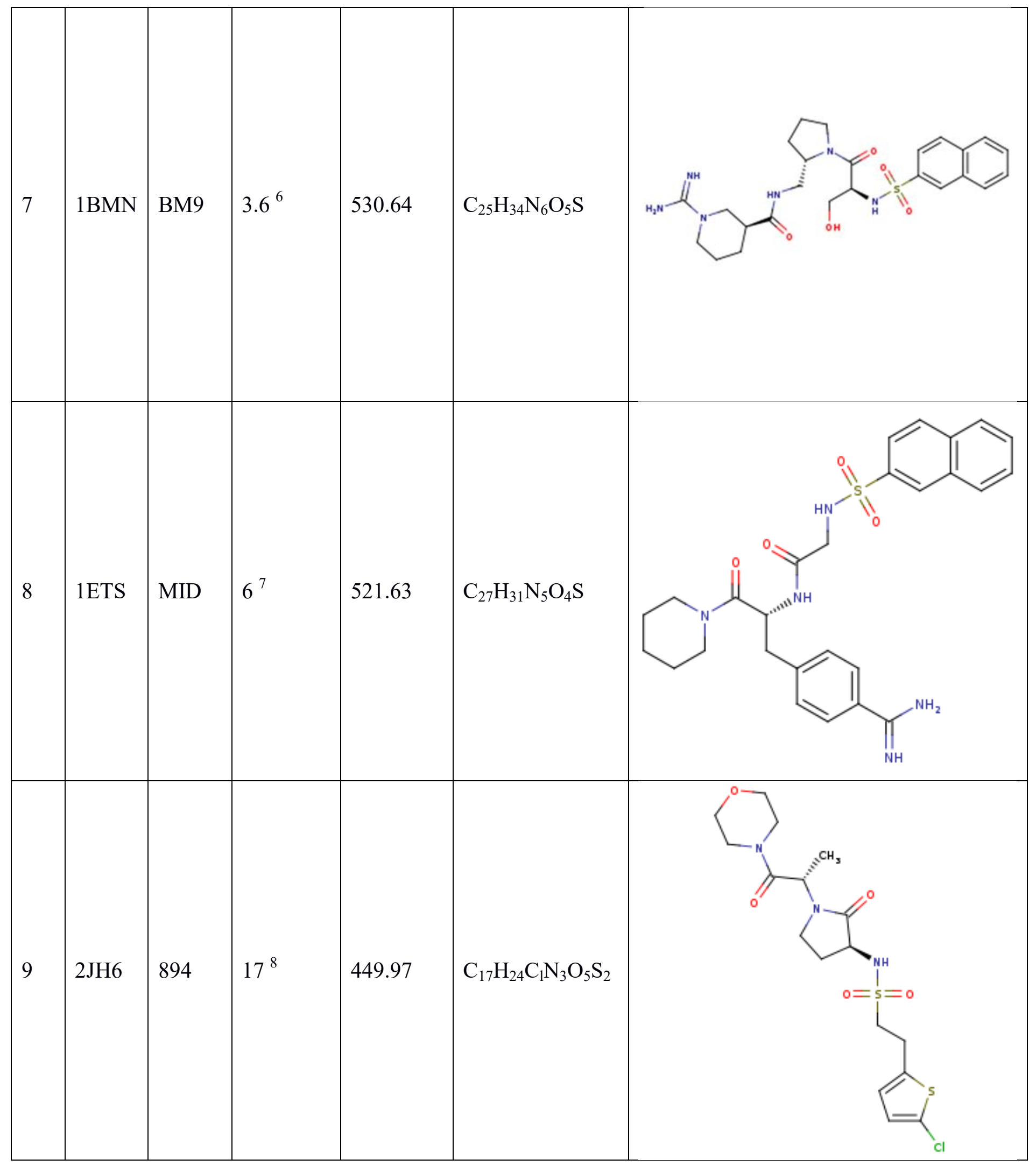




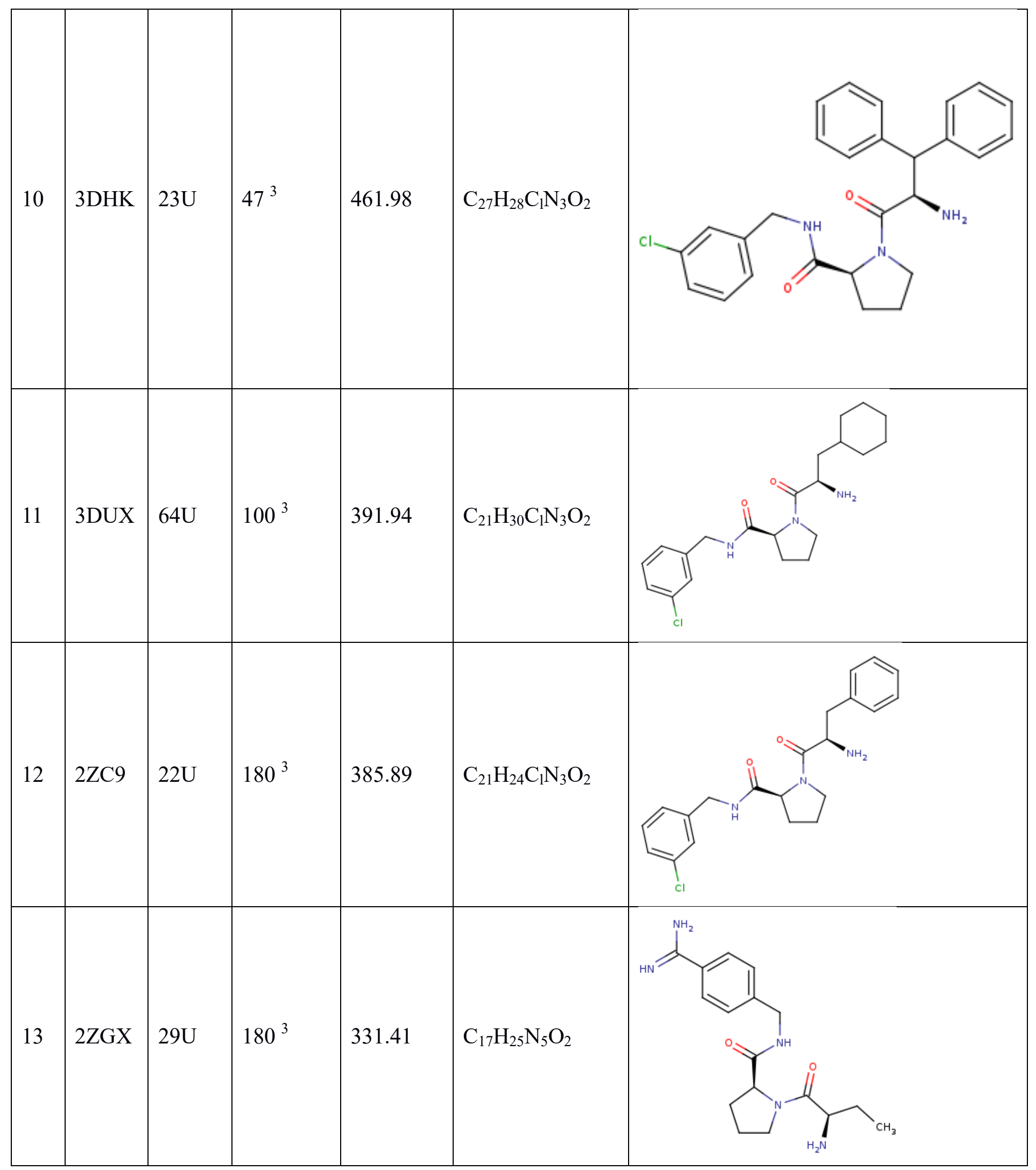




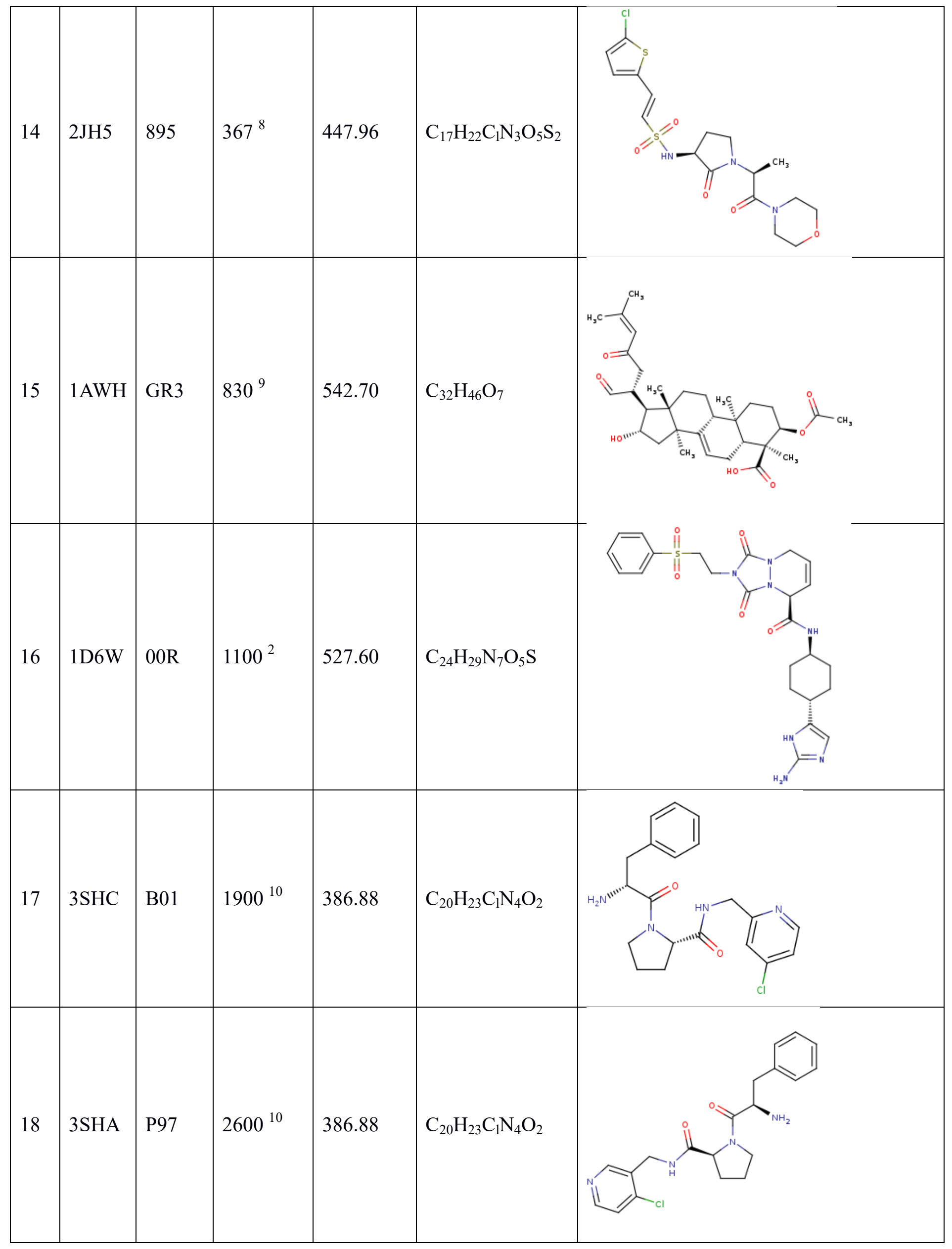




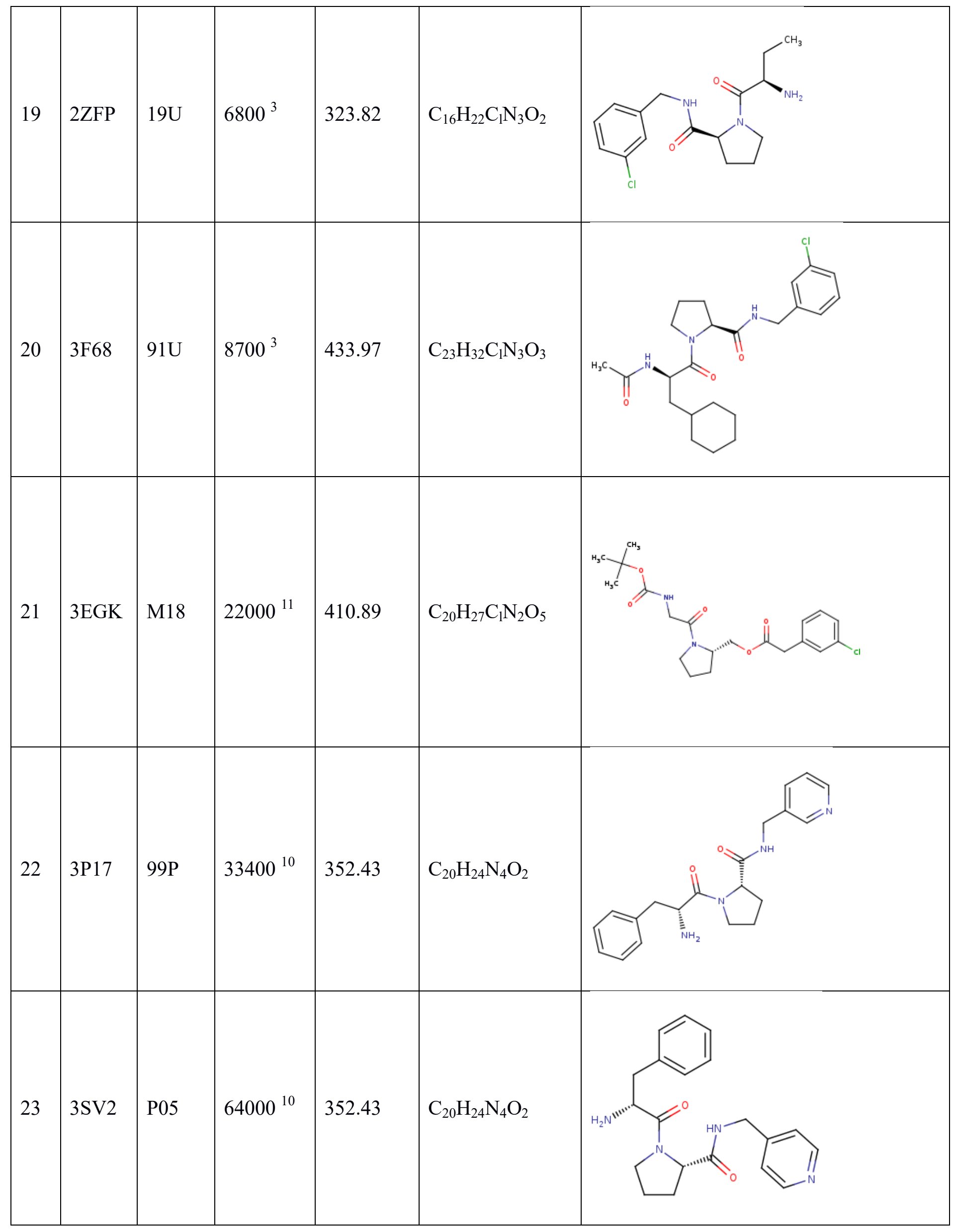


Table S2 - Inhibition constant, molecular weight, chemical formula and 2D structure of the 23 studied ligands of factor Xa. Their ID is given in the third column, while the second column refers to PDB ID of their complex with factor Xa protein.

\begin{tabular}{|c|c|c|c|c|c|c|}
\hline No. & $\begin{array}{l}\text { PDB } \\
\text { ID }\end{array}$ & $\begin{array}{l}\text { Ligand } \\
\text { ID }\end{array}$ & $\begin{array}{l}\text { Inhibition } \\
\text { constant } \\
\mathrm{Ki}(\mathrm{nm}) \\
\end{array}$ & $\begin{array}{l}\text { Molecular } \\
\text { weight } \\
(\mathrm{g} / \mathrm{mol})\end{array}$ & $\begin{array}{l}\text { Chemical } \\
\text { formula }\end{array}$ & 2D structure \\
\hline 1 & 2P3T & 993 & $0.005^{12}$ & 565.68 & $\mathrm{C}_{23} \mathrm{H}_{19} \mathrm{C}_{13} \mathrm{~N}_{6} \mathrm{O}_{3} \mathrm{~S}$ & \\
\hline 2 & 1MQ6 & XLD & $0.007^{13}$ & 568.86 & $\mathrm{C}_{23} \mathrm{H}_{20} \mathrm{C}_{13} \mathrm{~N}_{5} \mathrm{O}_{4} \mathrm{~S}$ & \\
\hline 3 & $3 \mathrm{CS} 7$ & LG0 & $0.021^{14}$ & 510.55 & $\mathrm{C}_{28} \mathrm{H}_{29} \mathrm{~F}_{3} \mathrm{~N}_{4} \mathrm{O}_{2}$ & \\
\hline 4 & $2 \mathrm{FZZ}$ & $5 \mathrm{QC}$ & $0.03^{15}$ & 588.58 & $\mathrm{C}_{31} \mathrm{H}_{27} \mathrm{~F}_{3} \mathrm{~N}_{6} \mathrm{O} 3$ & \\
\hline
\end{tabular}




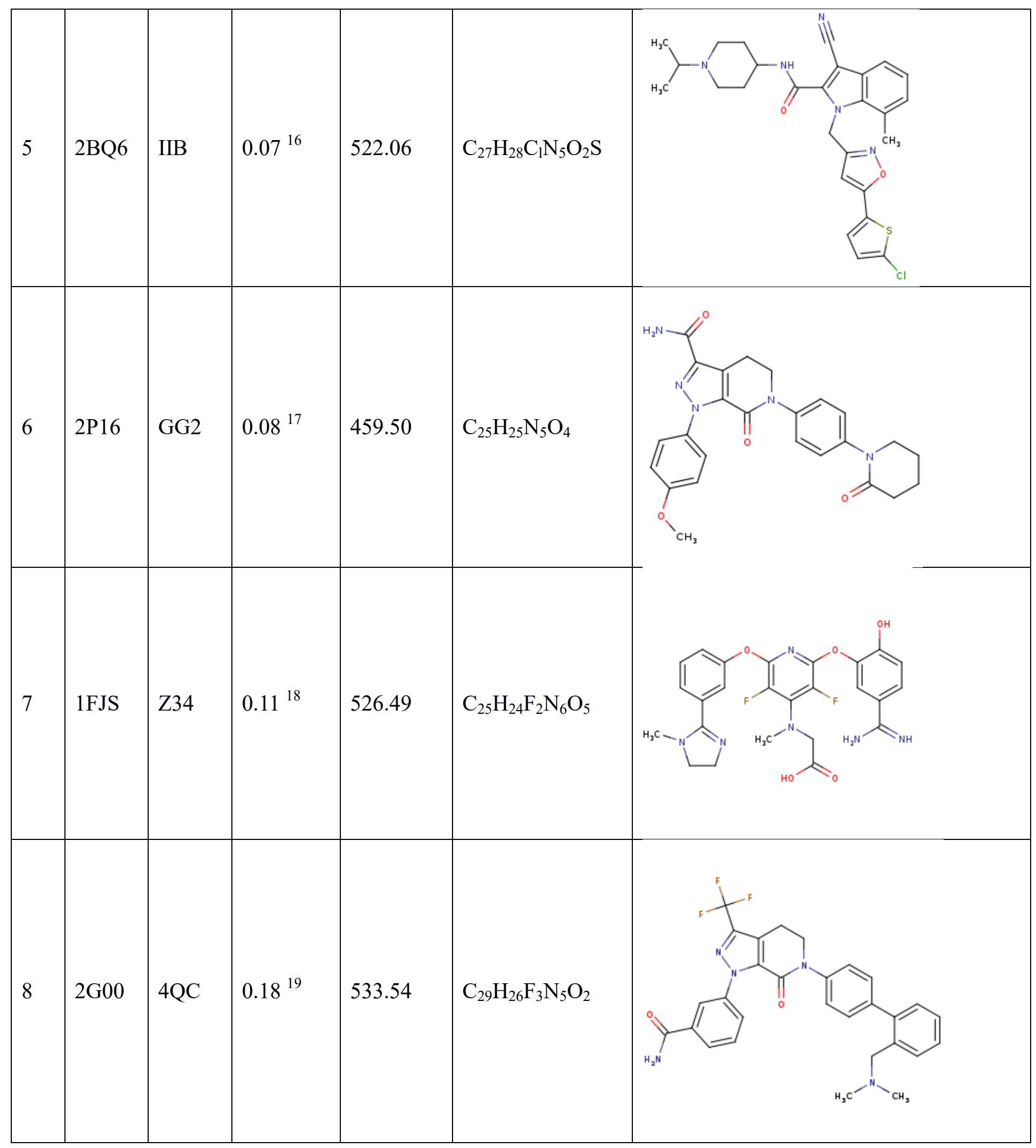




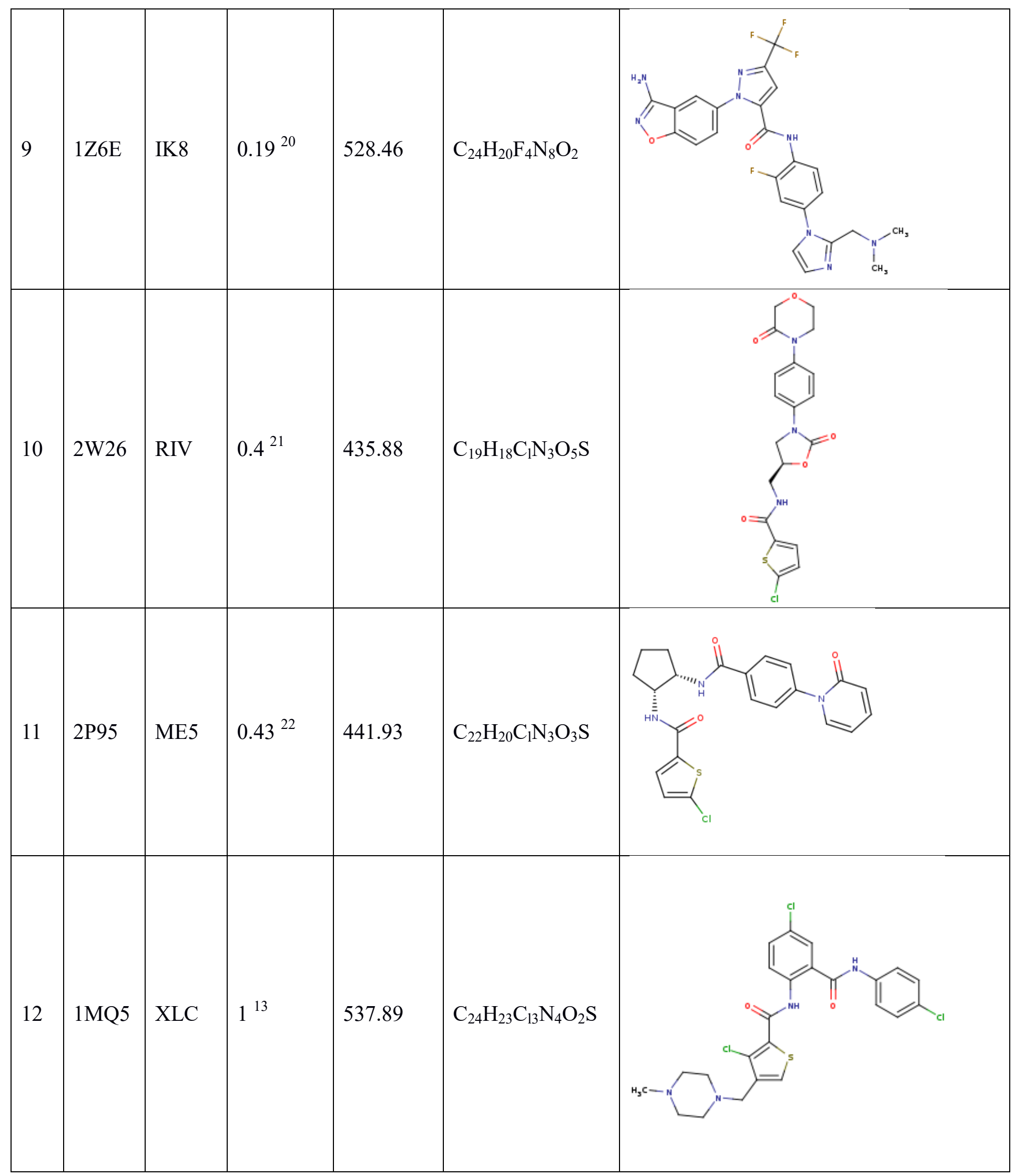




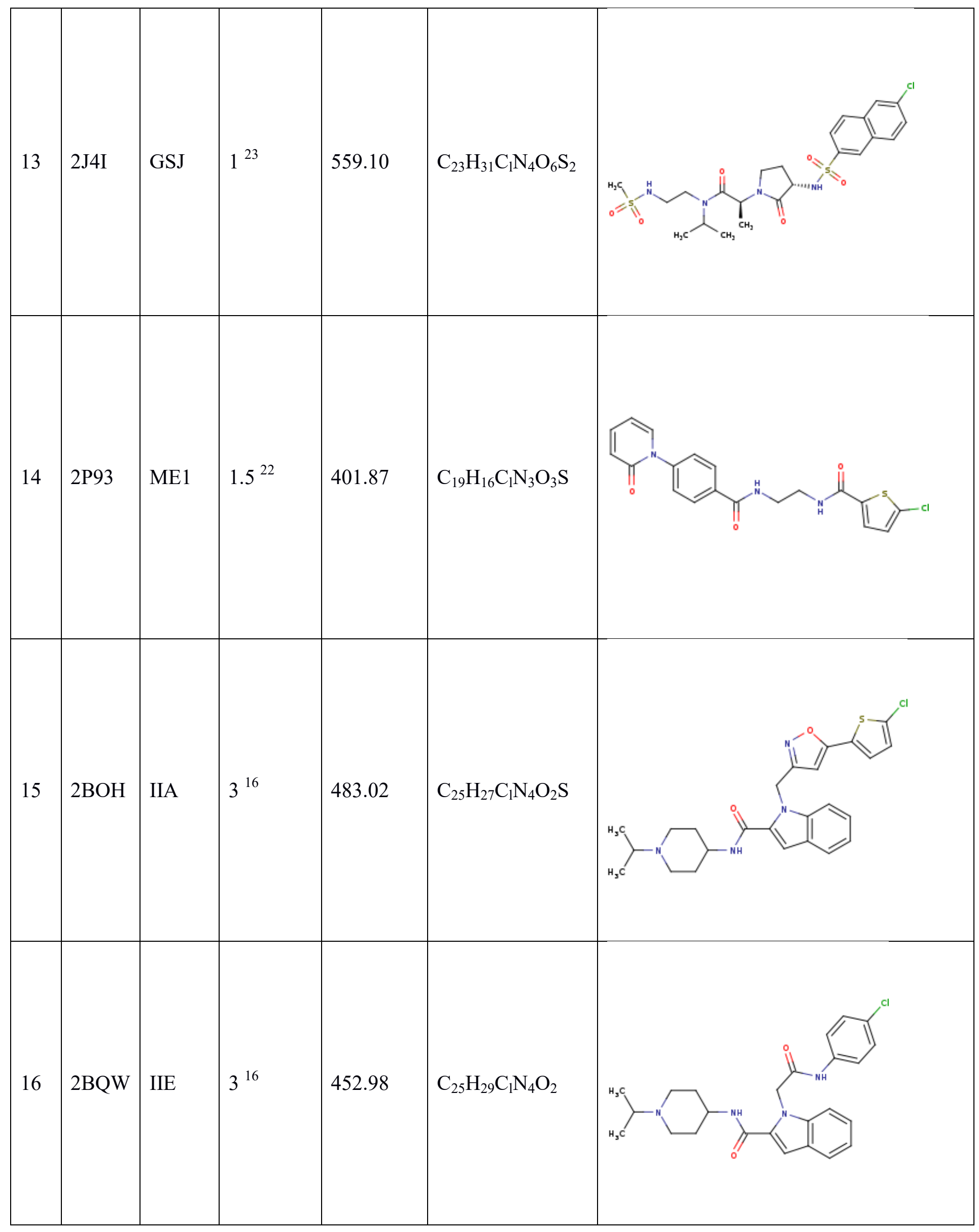




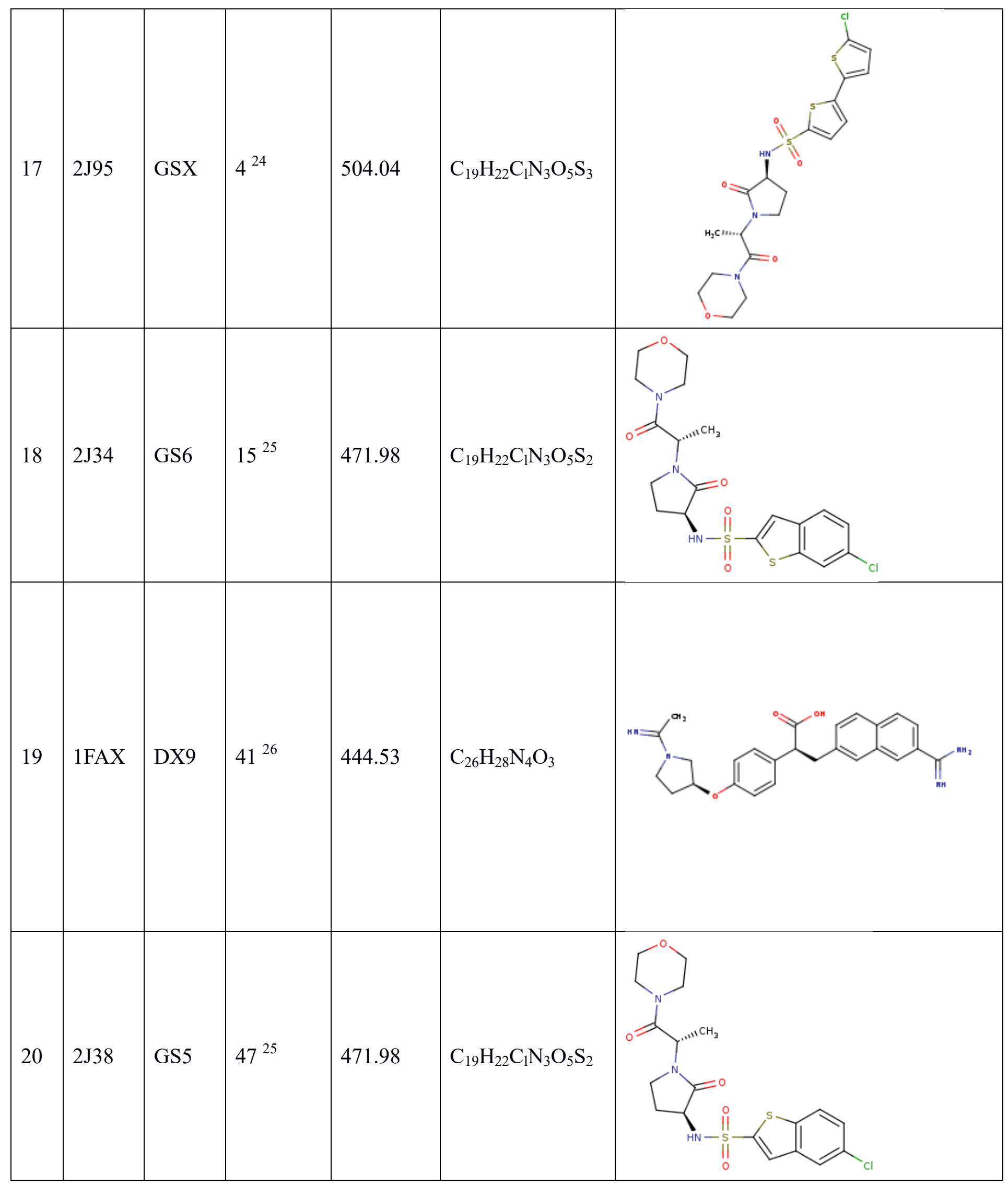




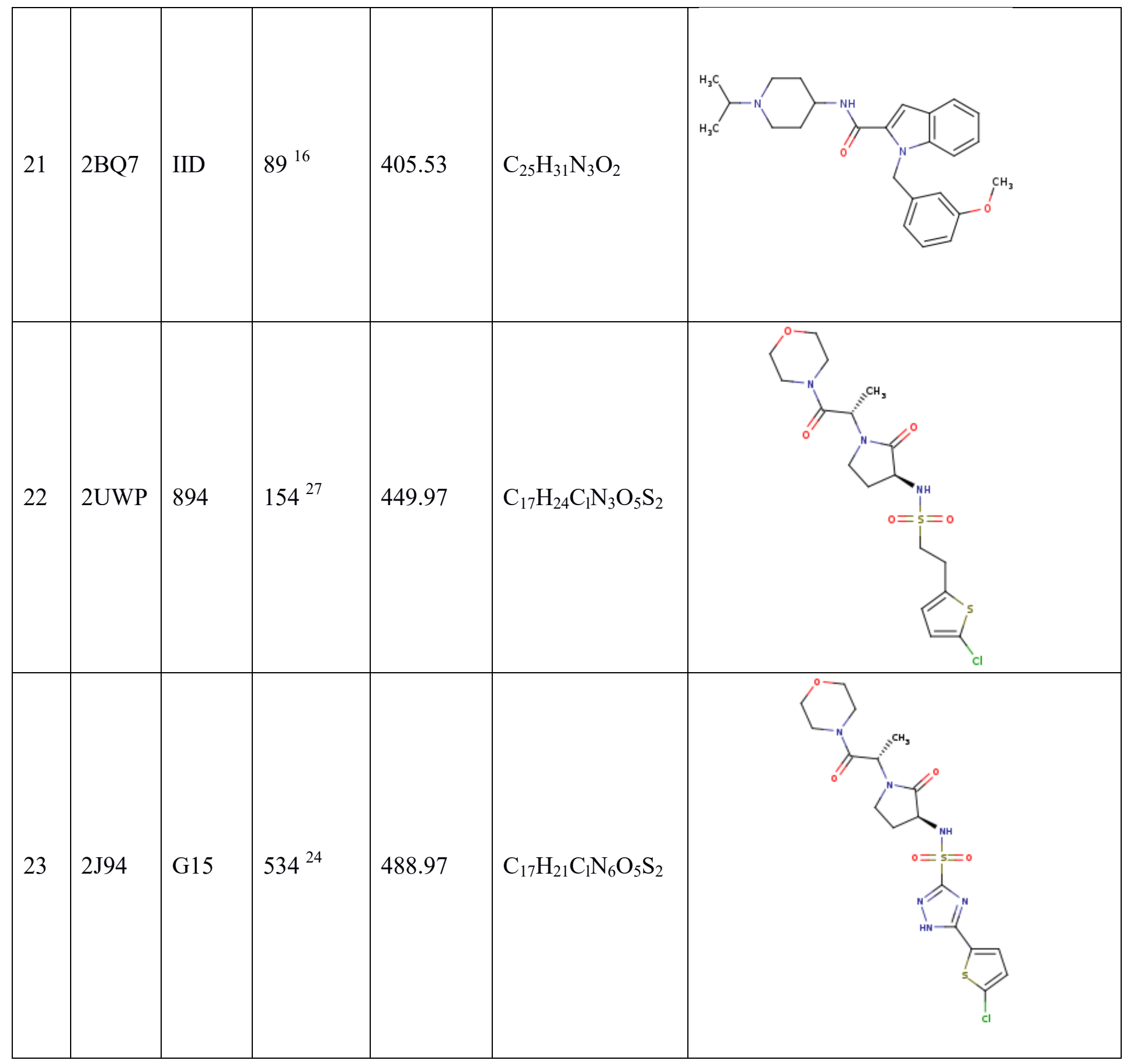

Table S3 - Inhibition constant, molecular weight, chemical formula and 2D structure of 23 studied ligands of HIV-1. Their ID is given in the third column, while the second column refers to PDB ID of their complex with the HIV-1 protein.

\begin{tabular}{|l|l|l|l|l|l|l|}
\hline No. & $\begin{array}{l}\text { PDB } \\
\text { ID }\end{array}$ & $\begin{array}{l}\text { Ligand } \\
\text { ID }\end{array}$ & $\begin{array}{l}\text { Inhibition } \\
\text { constant } \\
\mathrm{Ki}(\mathrm{nM})\end{array}$ & $\begin{array}{l}\text { Molecular } \\
\text { weight } \\
(\mathrm{g} / \mathrm{mol})\end{array}$ & $\begin{array}{l}\text { Chemical } \\
\text { formular }\end{array}$ & 2D structure \\
\hline
\end{tabular}




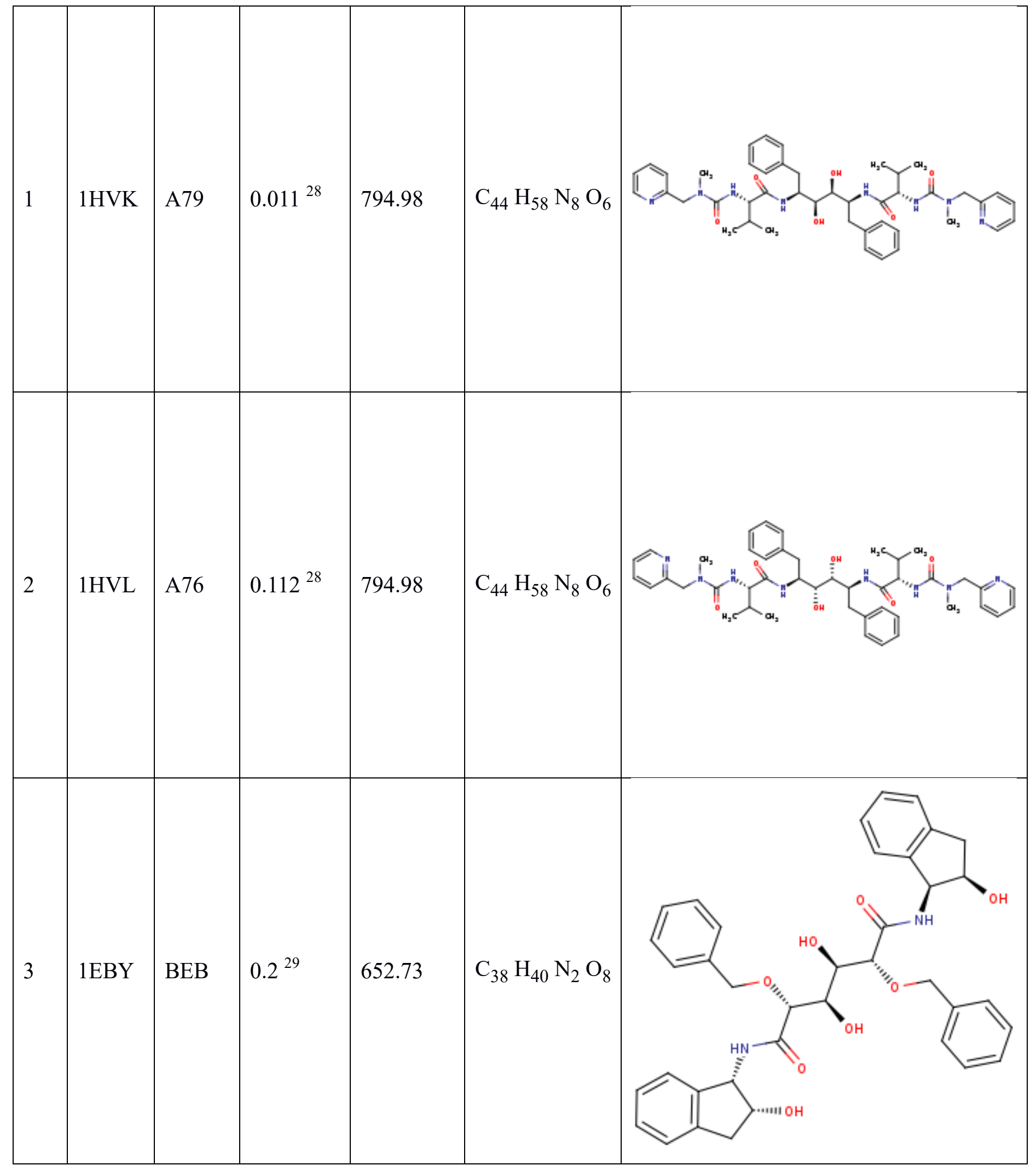




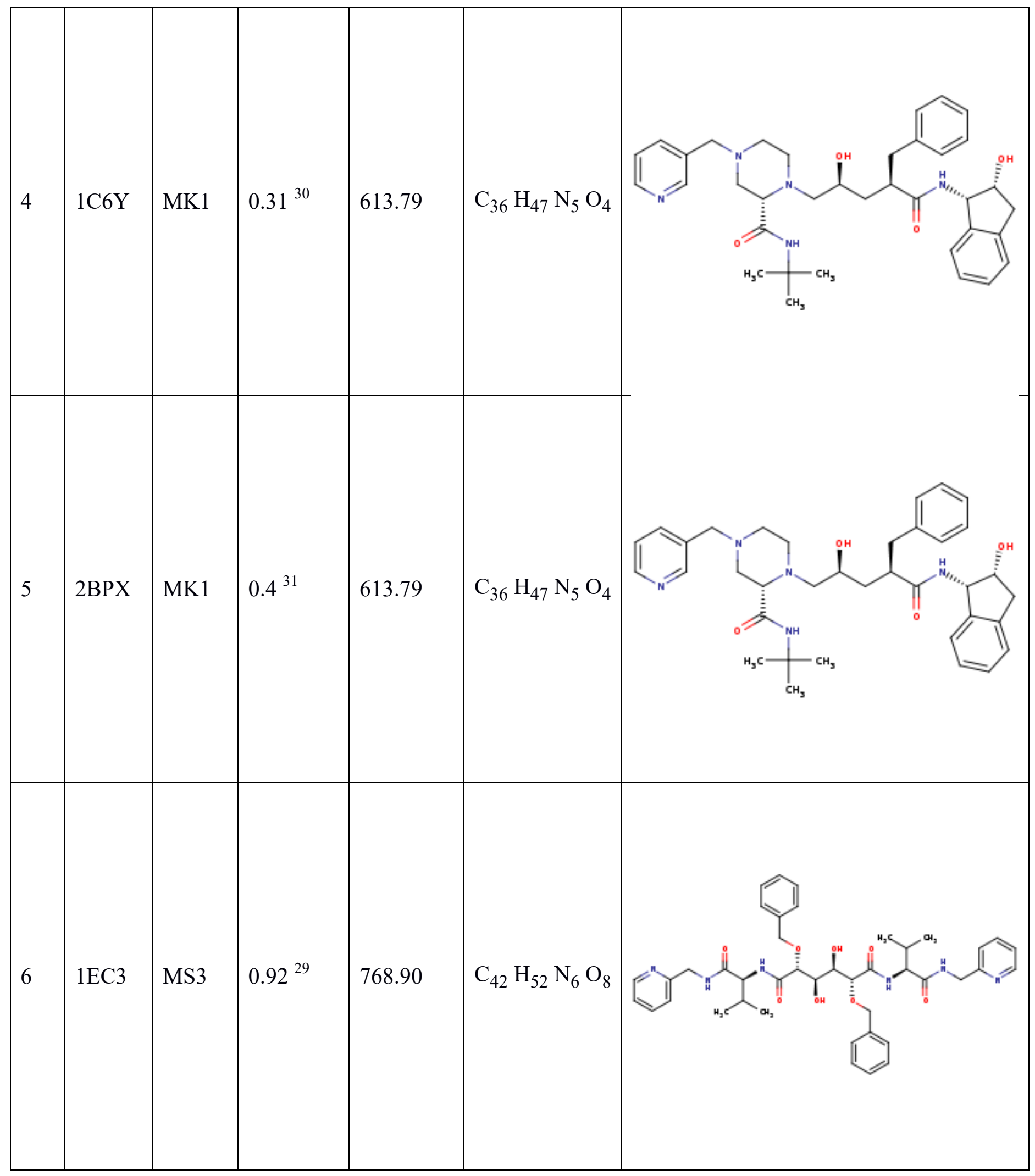

S14 


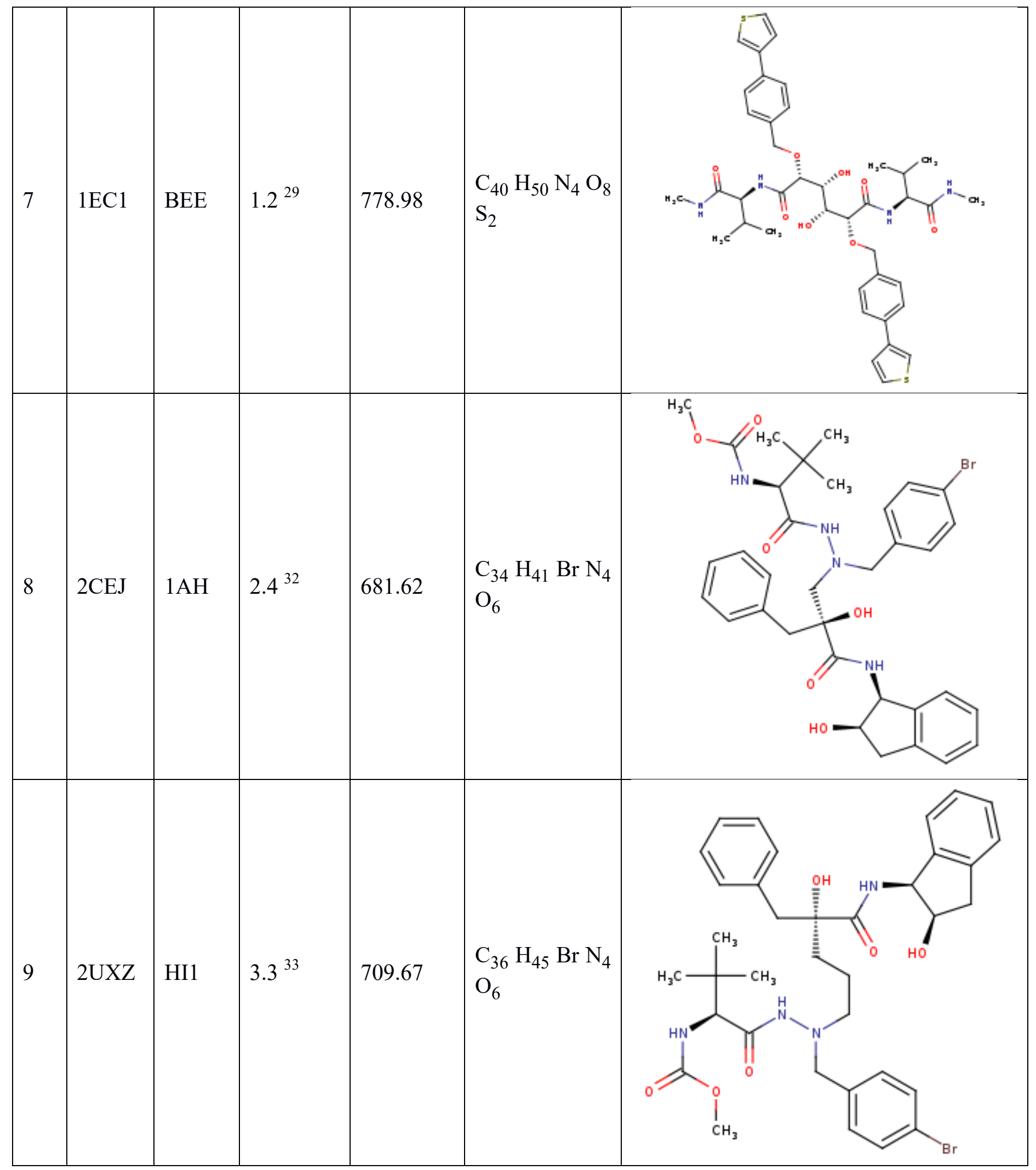




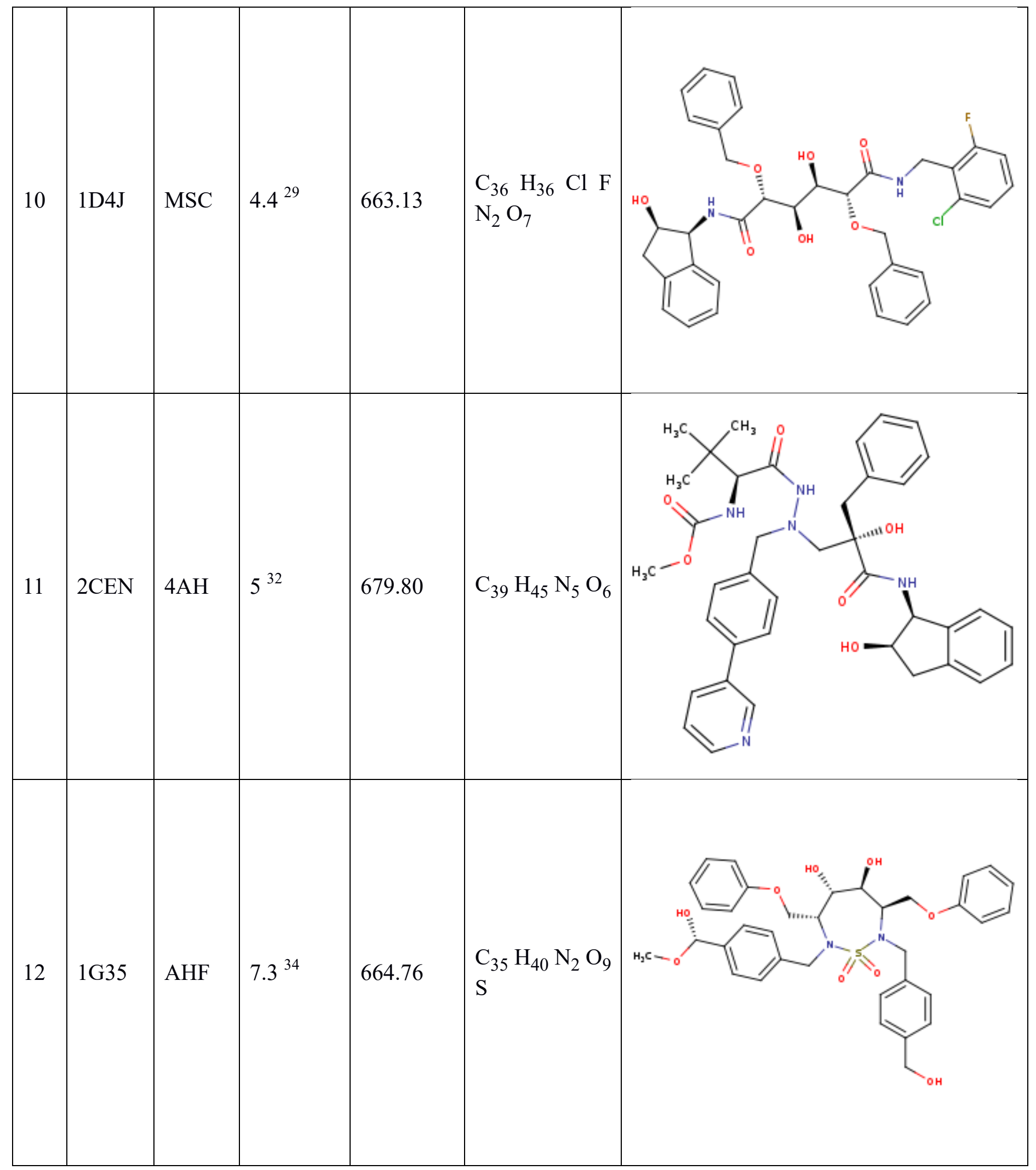




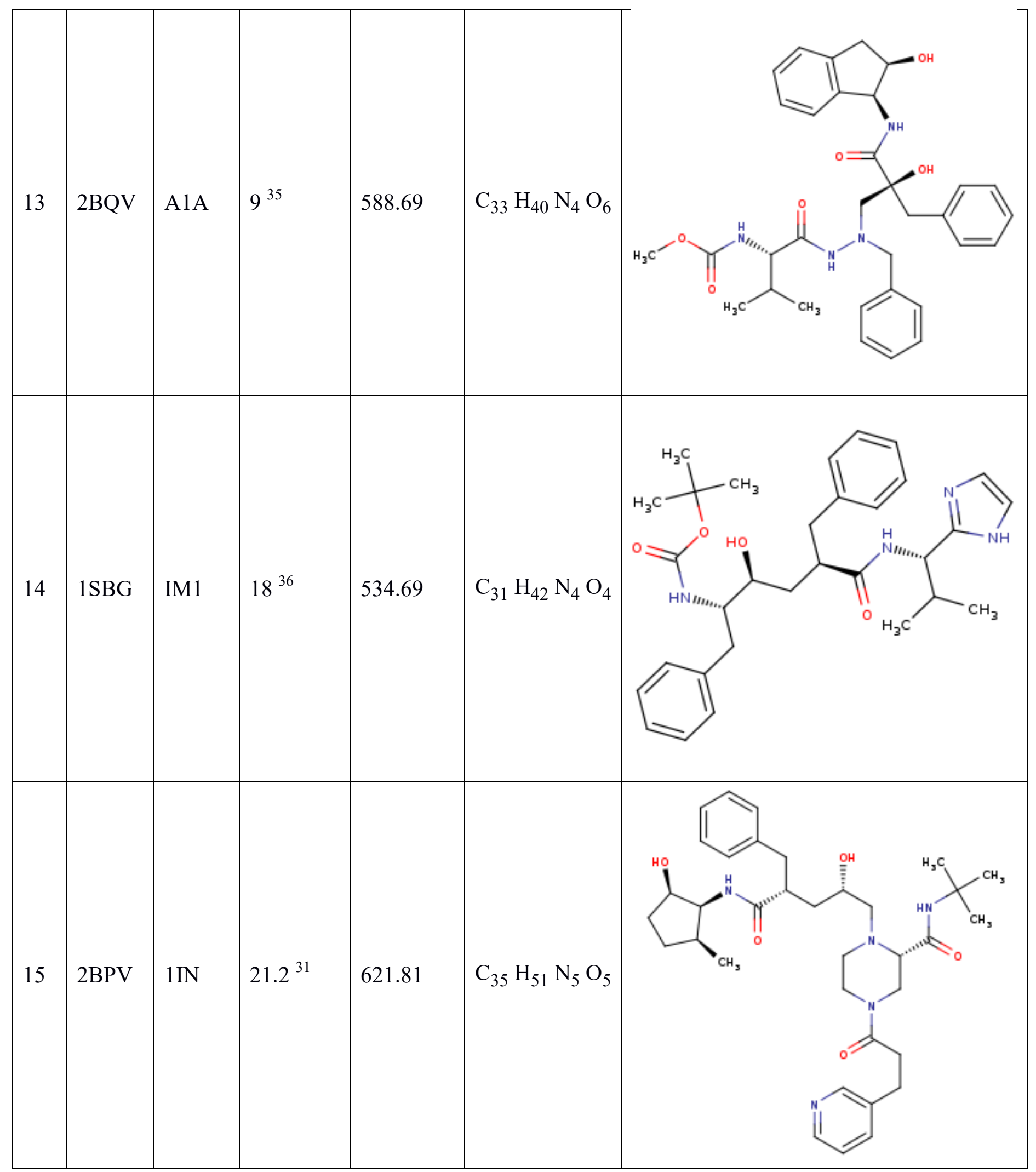




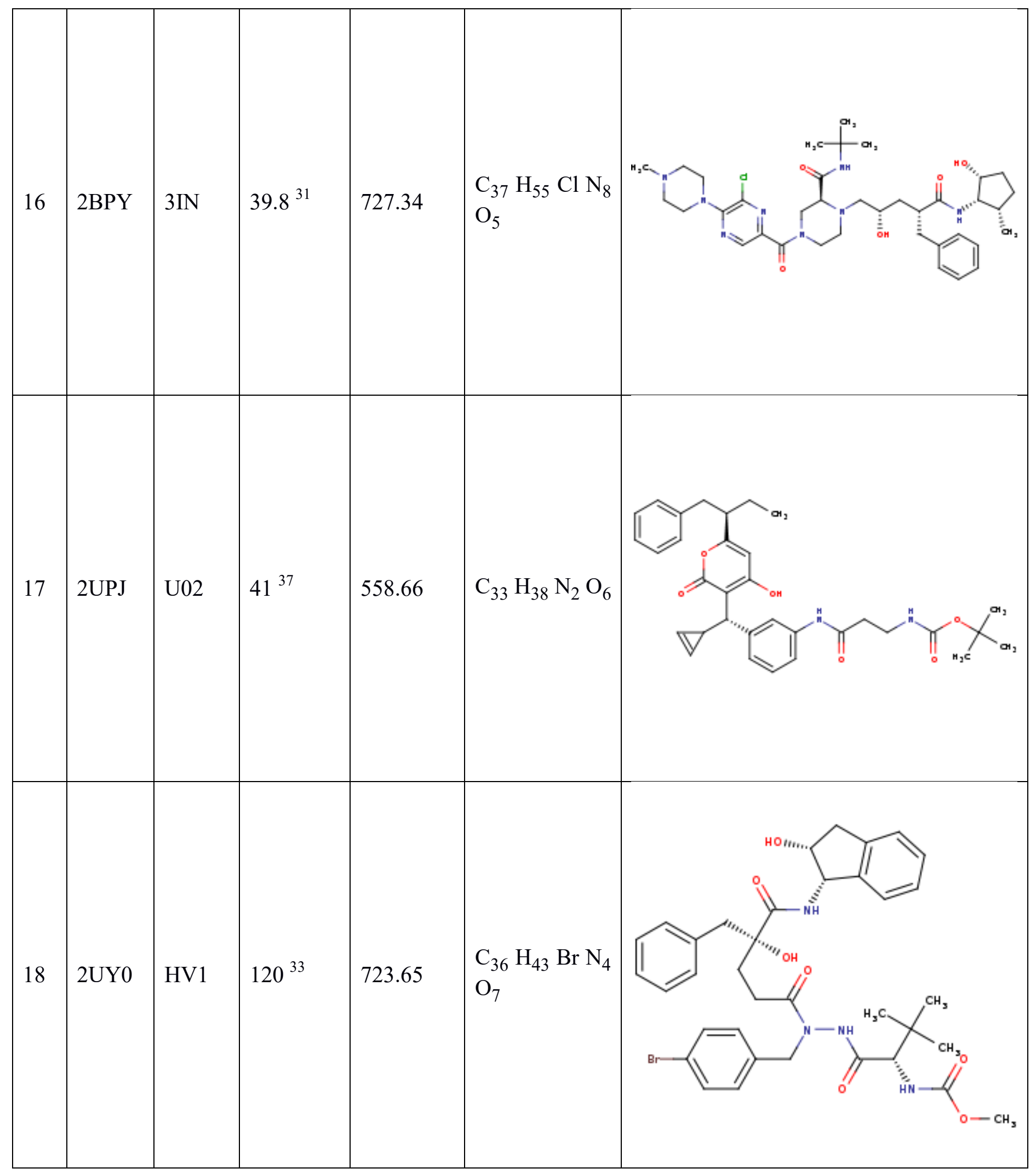




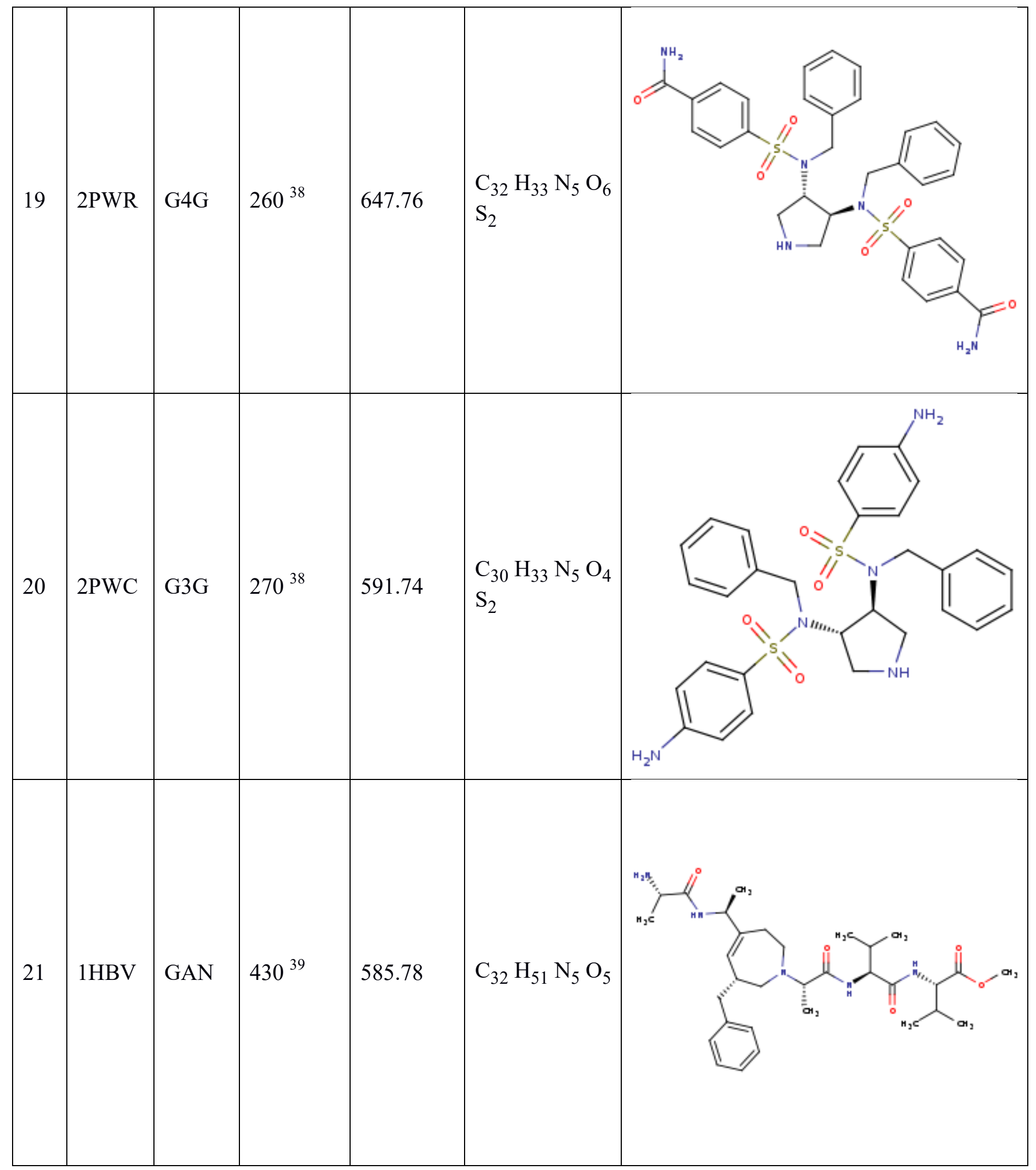




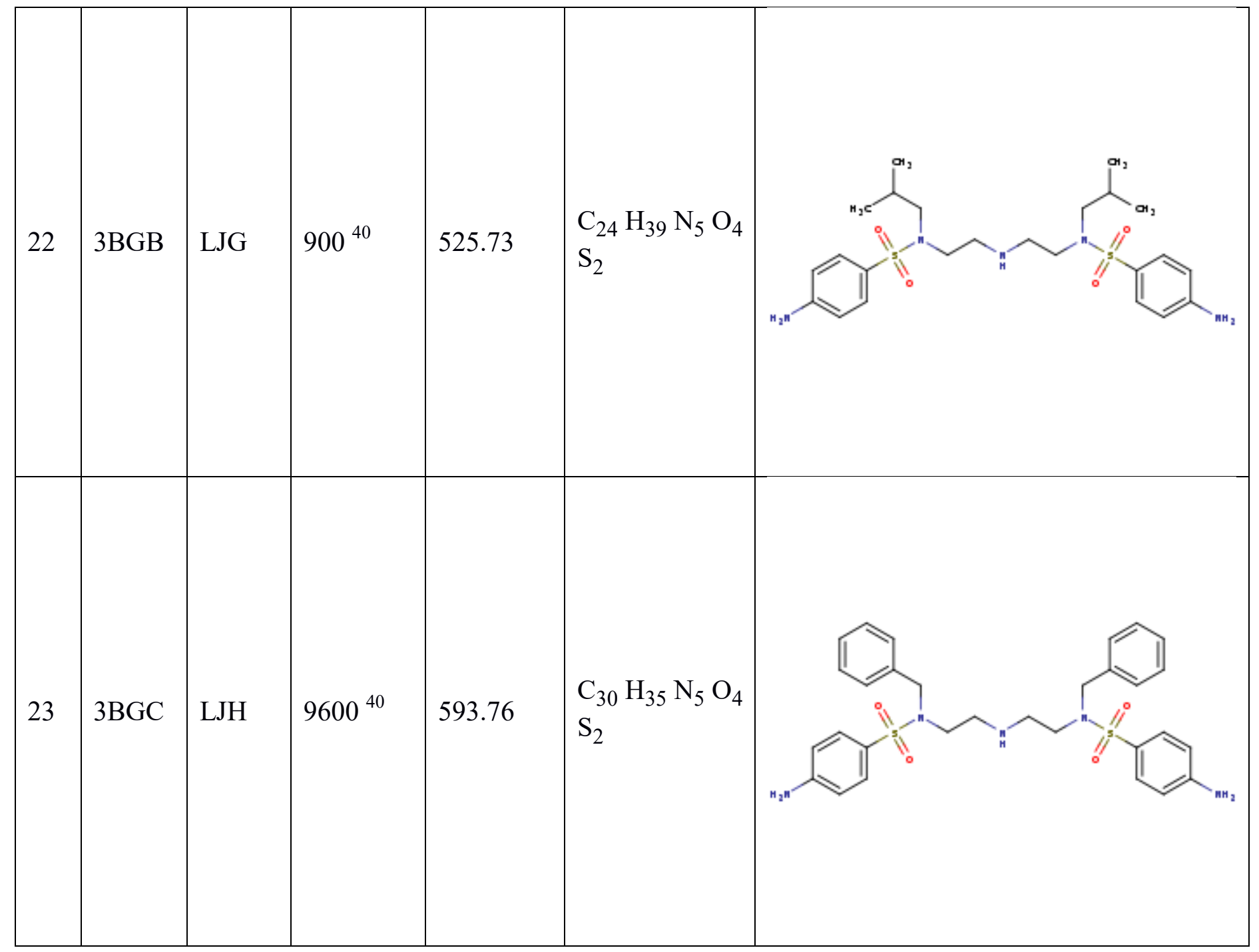

Table S4 - Inhibition constant, molecular weight, chemical formula and 2D structure of 23 studied ligands of MCL-1. Their ID is given in the third column, while the second column refers to PDB ID of their complex with the mcl-1 protein.

\begin{tabular}{|l|l|l|l|l|l|l|}
\hline No. & $\begin{array}{l}\text { PDB } \\
\text { ID }\end{array}$ & $\begin{array}{l}\text { Ligand } \\
\text { ID }\end{array}$ & $\begin{array}{l}\text { Inhibition } \\
\text { constant } \\
\mathrm{Ki}(\mathrm{nM})\end{array}$ & $\begin{array}{l}\text { Molecular } \\
\text { weight } \\
(\mathrm{g} / \mathrm{mol})\end{array}$ & $\begin{array}{l}\text { Chemical } \\
\text { formular }\end{array}$ & 2D structure \\
\hline
\end{tabular}




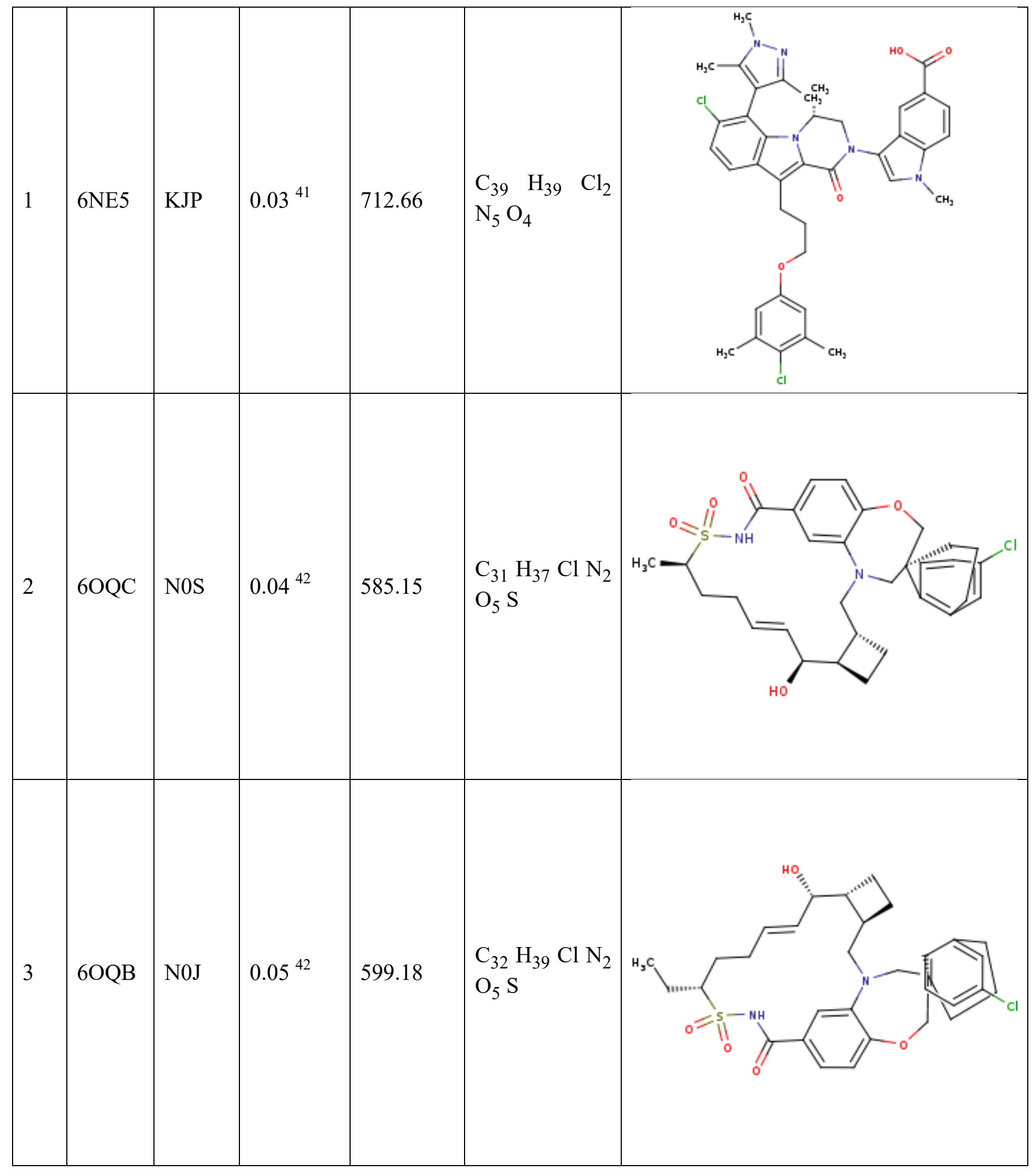




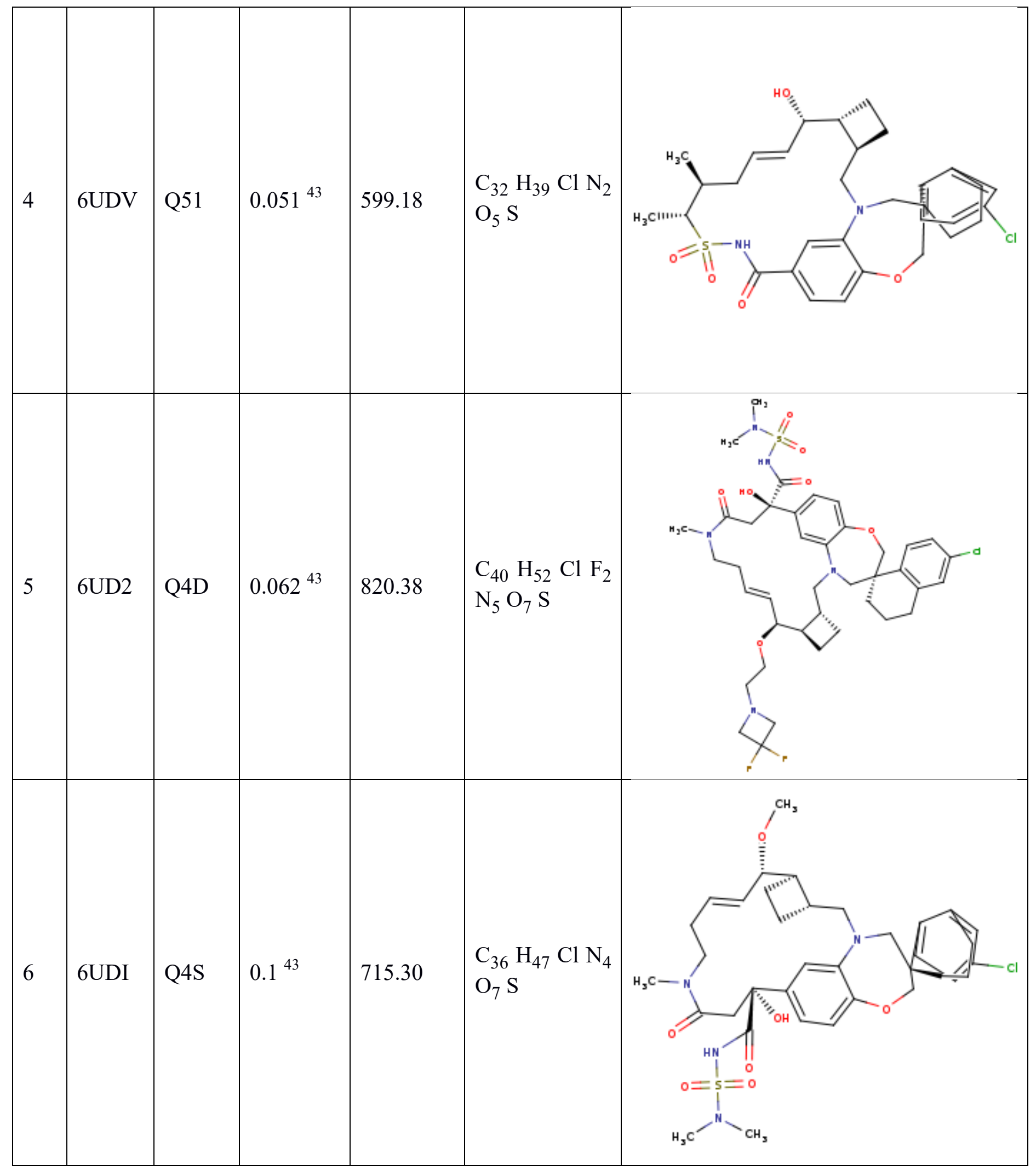




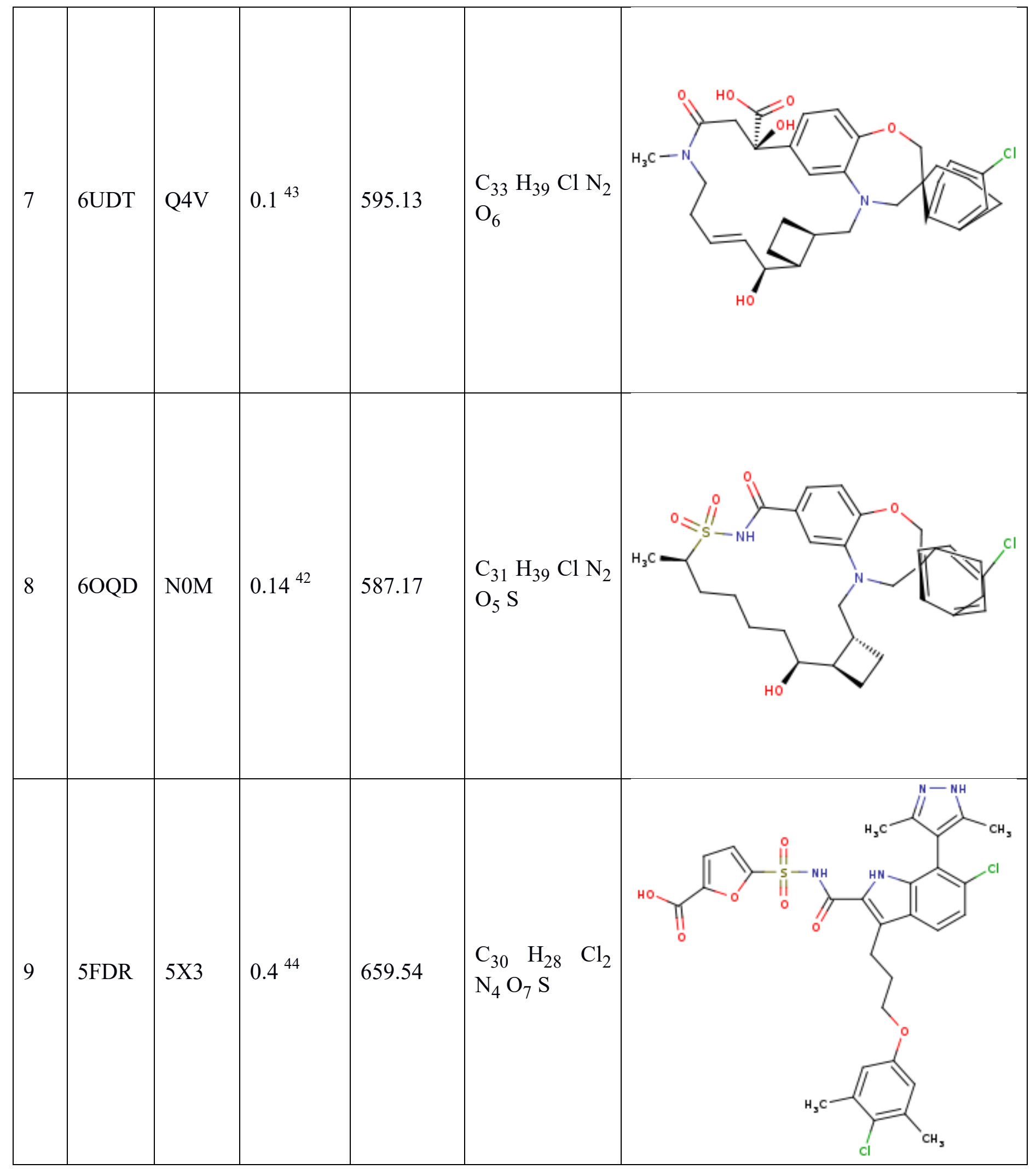




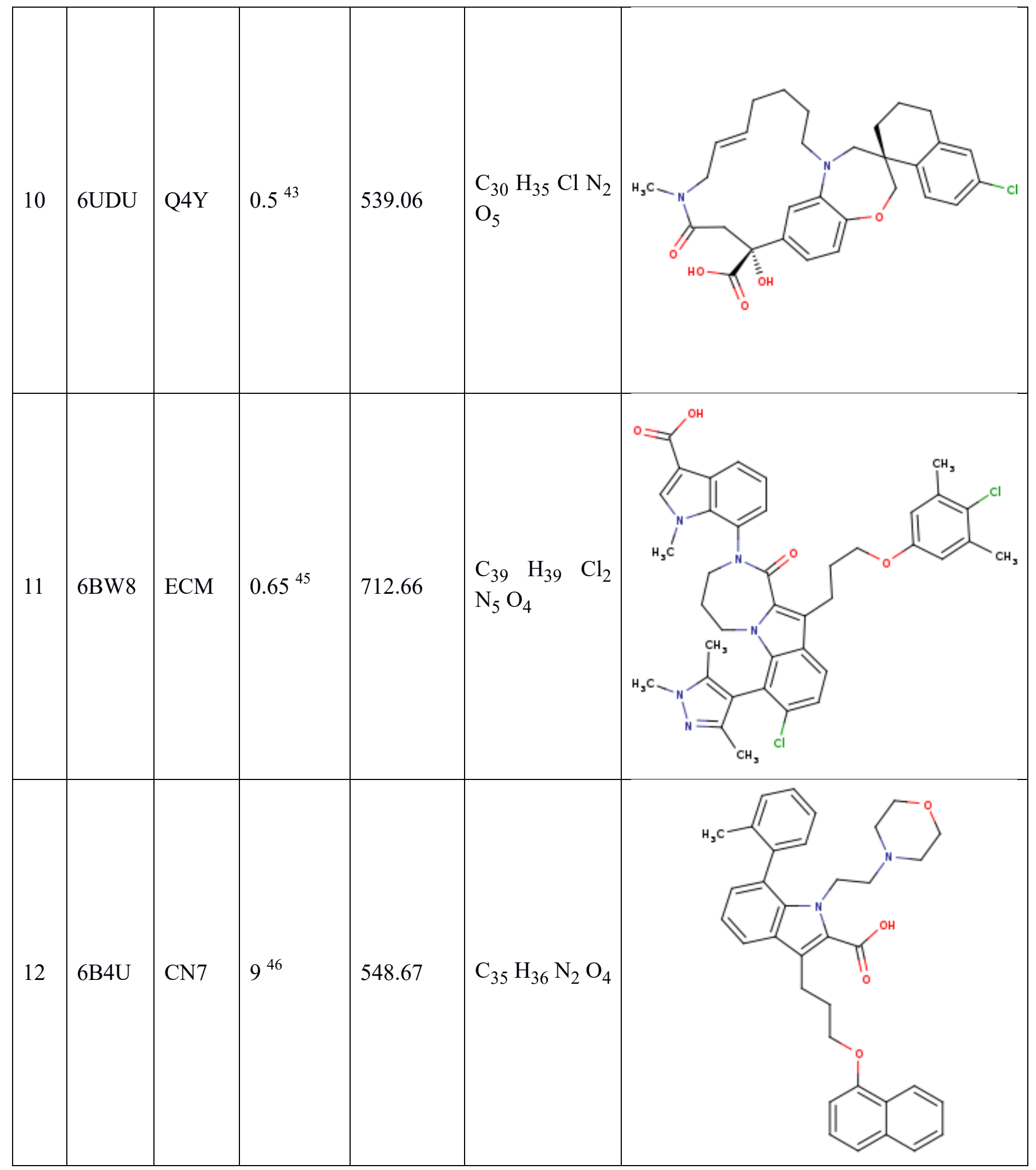




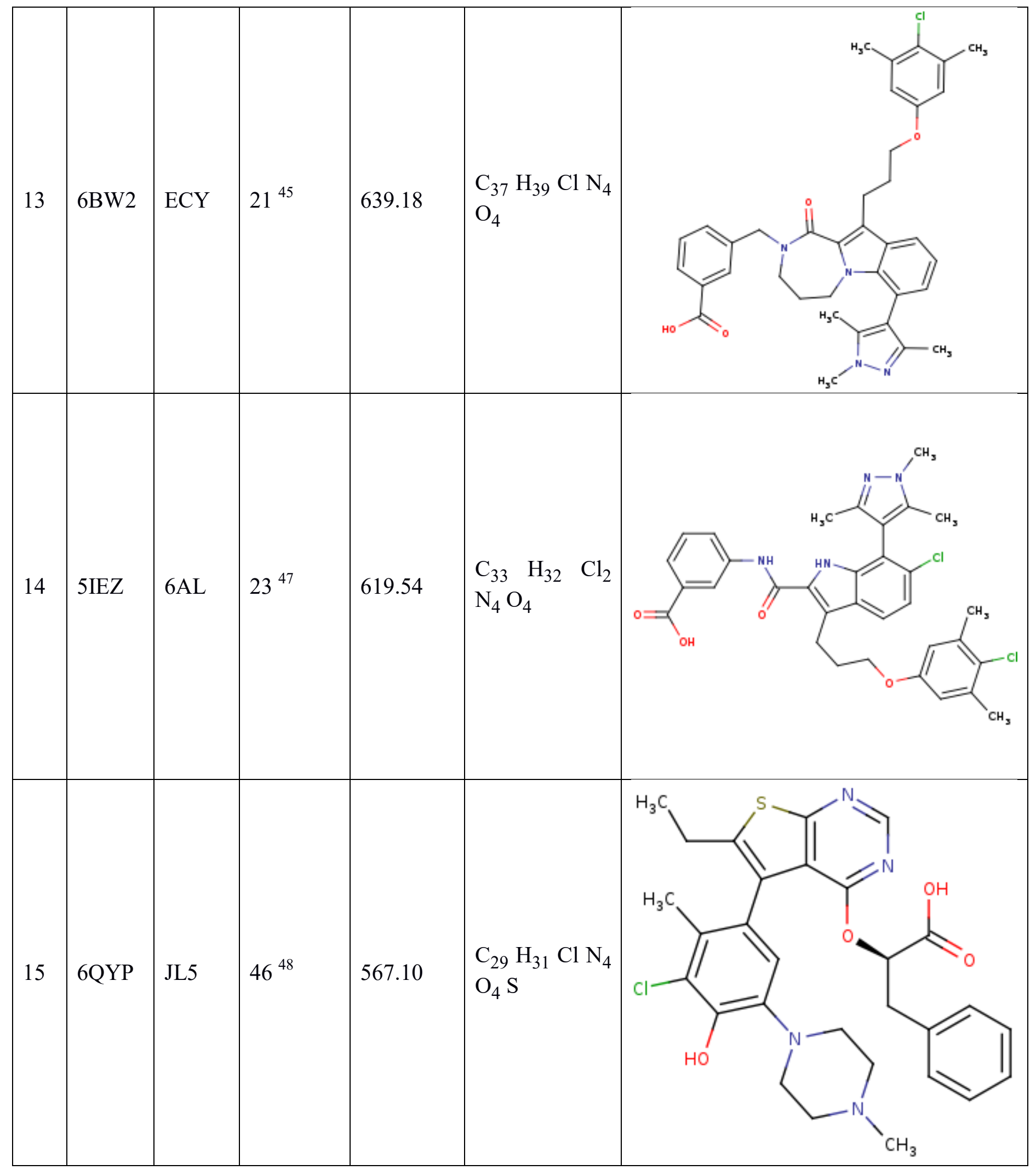




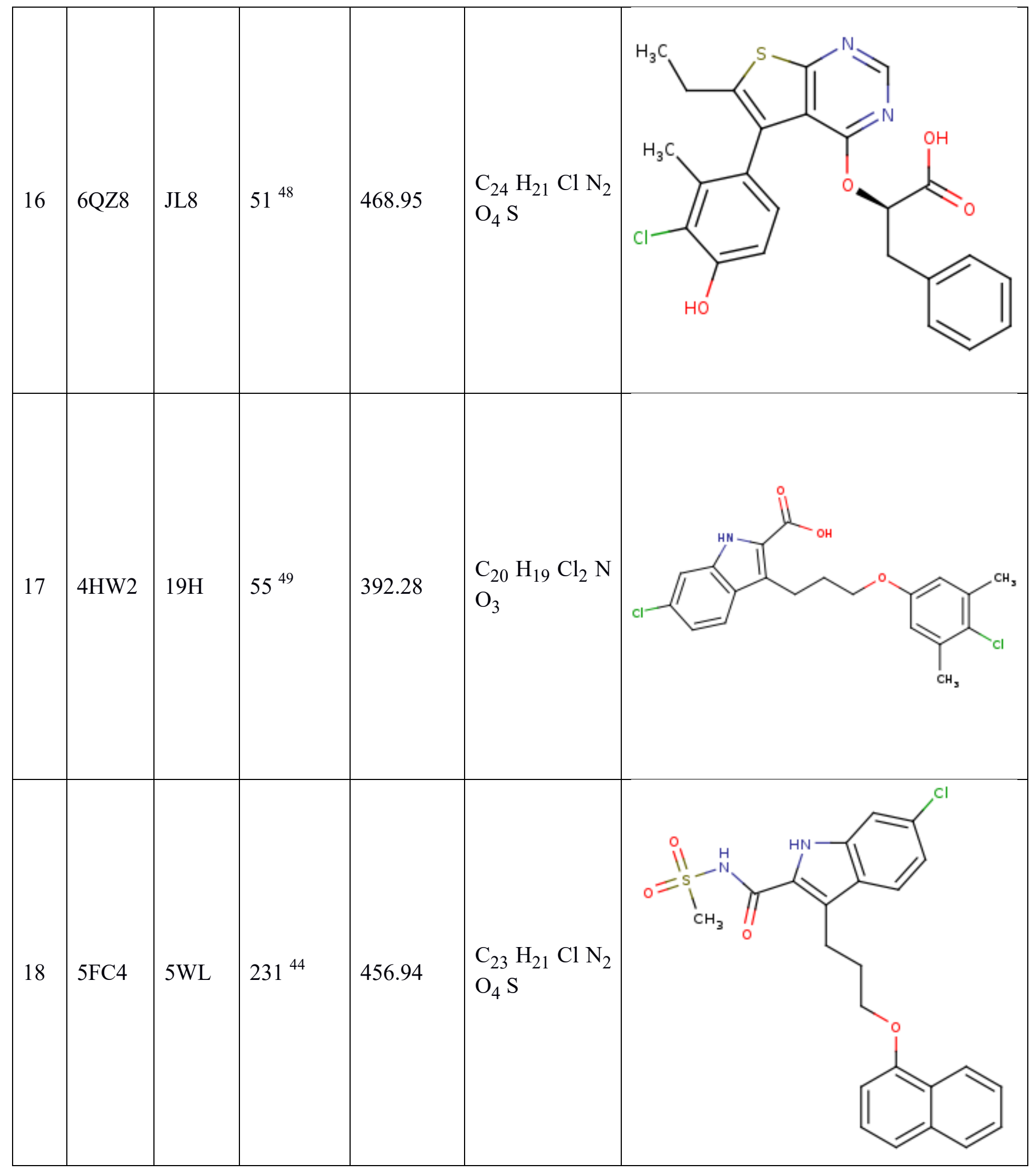




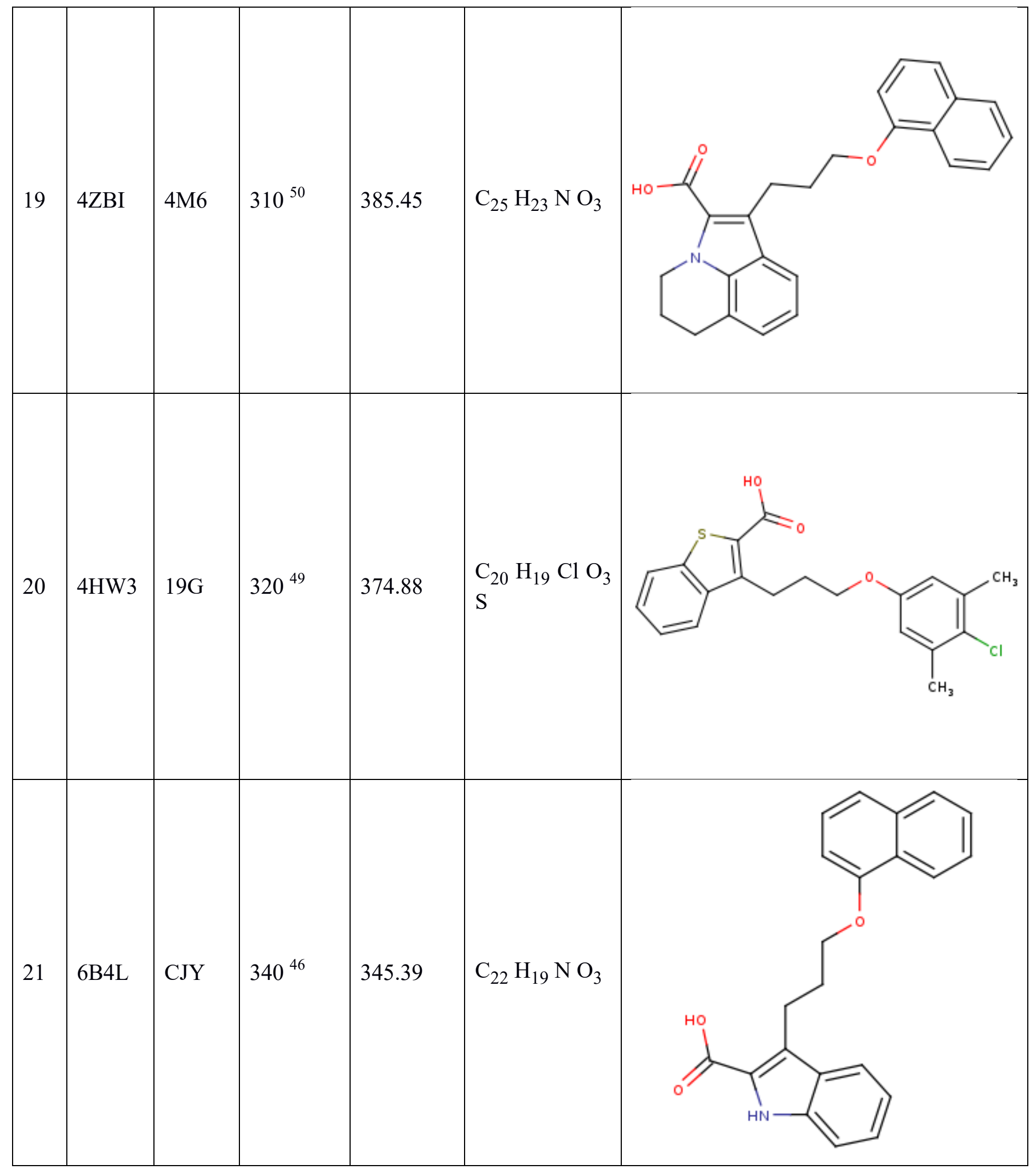




2205 FDO $561^{44}+497.01$

Table S5 - Inhibition constant, molecular weight, chemical formula and 2D structure of 23 studied ligands of CDK-2. Their ID is given in the third column, while the second column refers to PDB ID of their complex with the cdk-2 protein.

\begin{tabular}{|l|l|l|l|l|l|l|}
\hline No. & $\begin{array}{l}\text { PDB } \\
\text { ID }\end{array}$ & $\begin{array}{l}\text { Ligand } \\
\text { ID }\end{array}$ & $\begin{array}{l}\text { Inhibition } \\
\text { constant } \\
\text { Ki }(\mathrm{nM})\end{array}$ & $\begin{array}{l}\text { Molecular } \\
\text { weight } \\
(\mathrm{g} / \mathrm{mol})\end{array}$ & $\begin{array}{l}\text { Chemical } \\
\text { formular }\end{array}$ & 2D structure \\
\hline
\end{tabular}




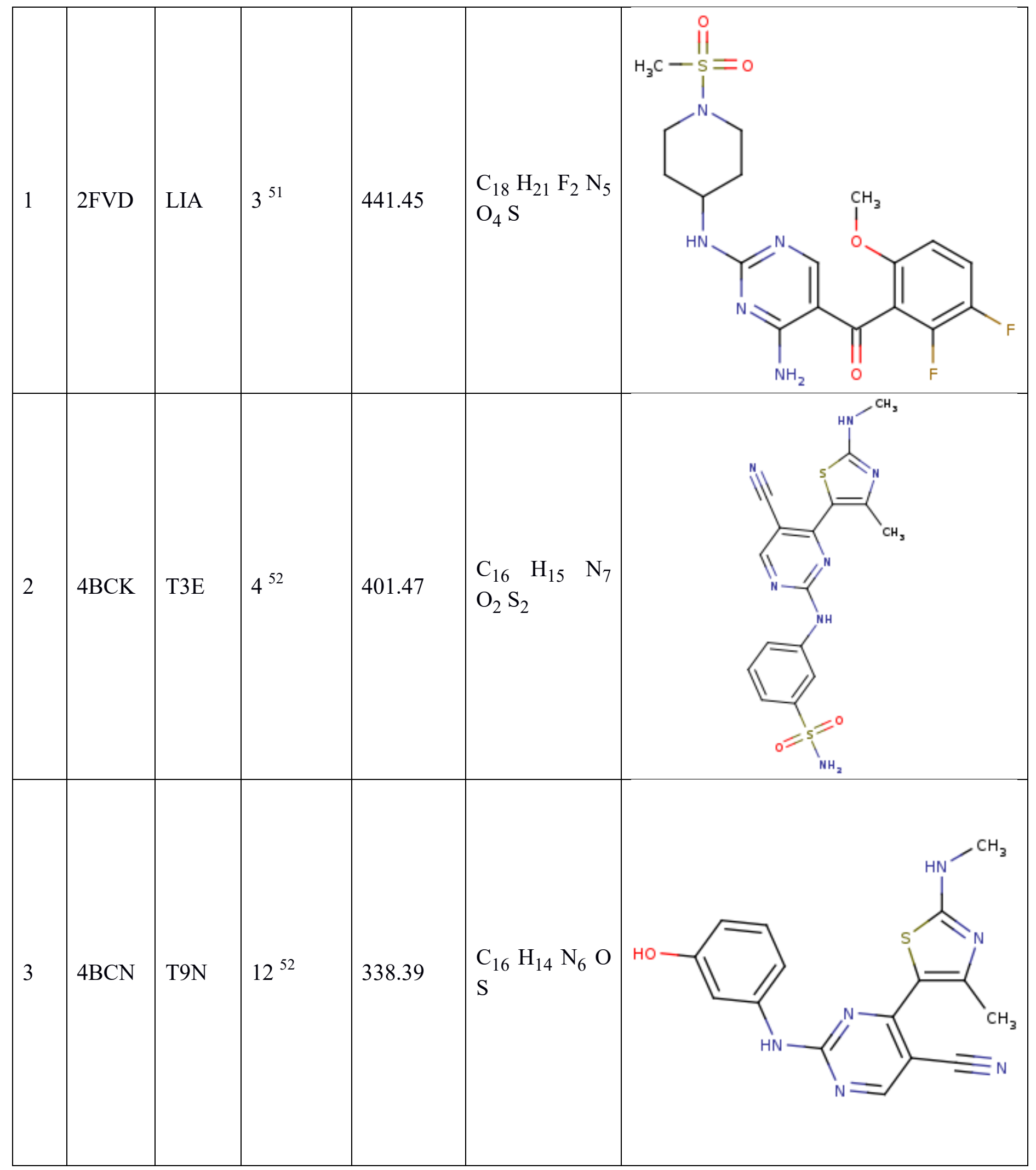




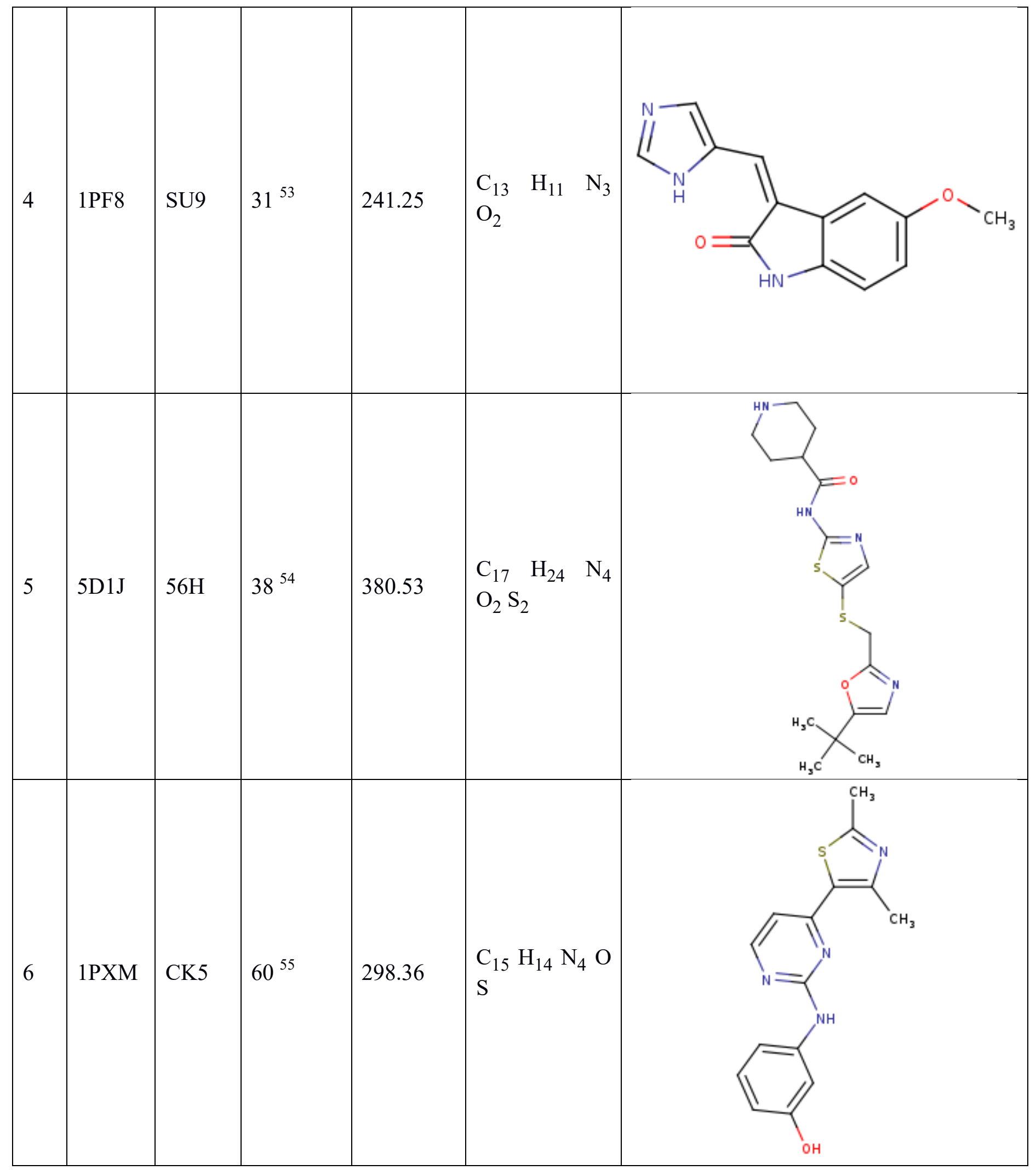




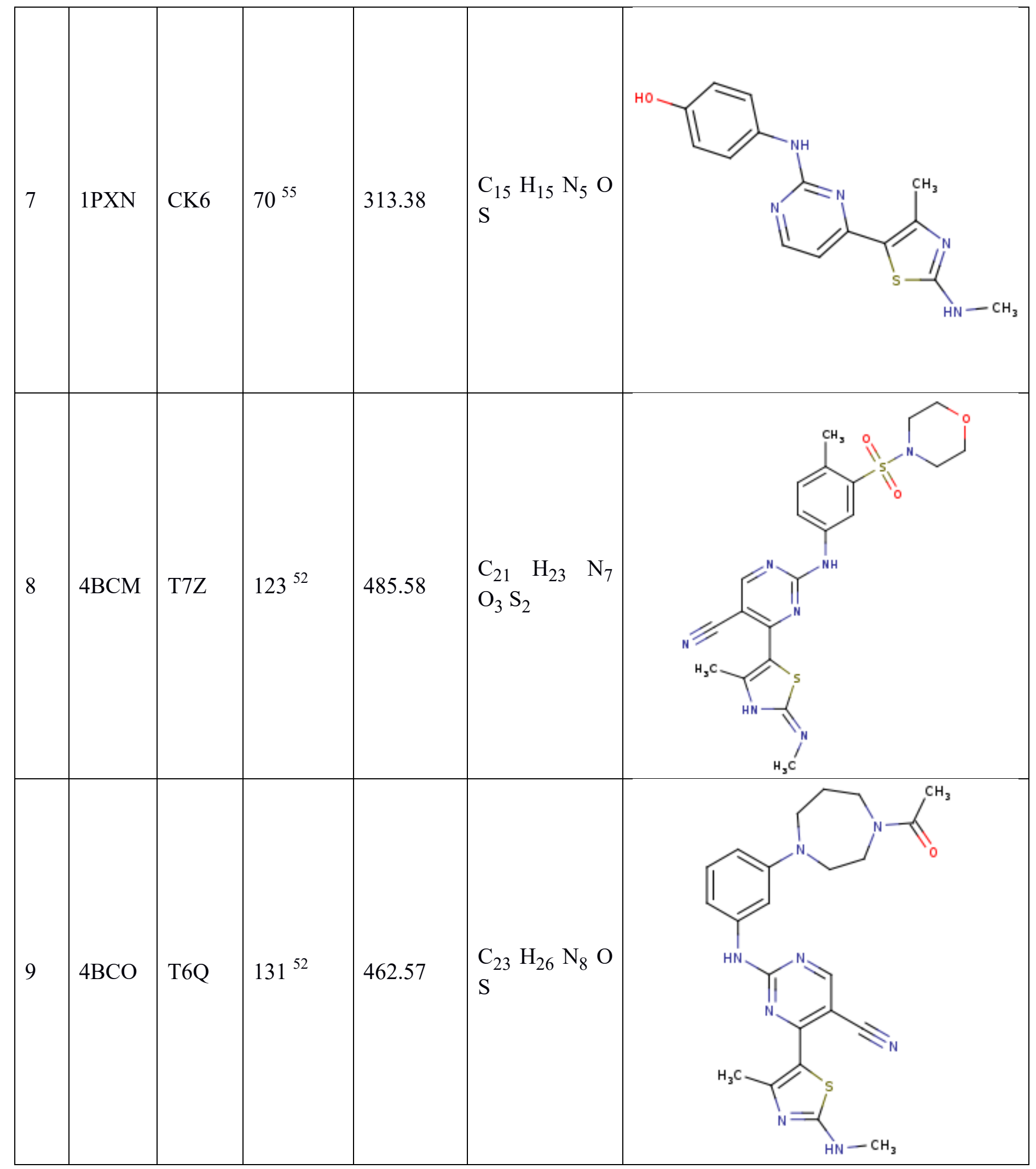




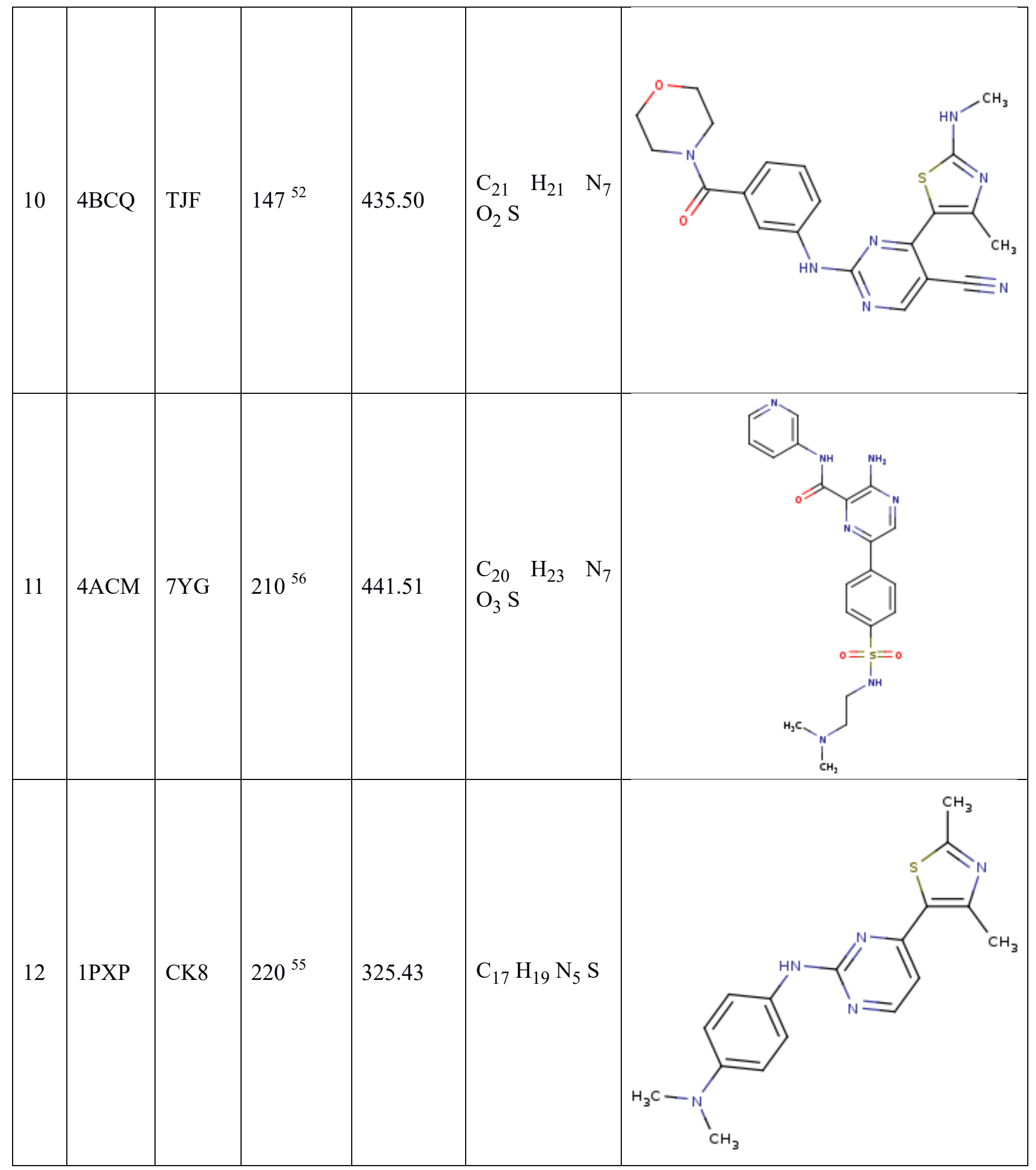




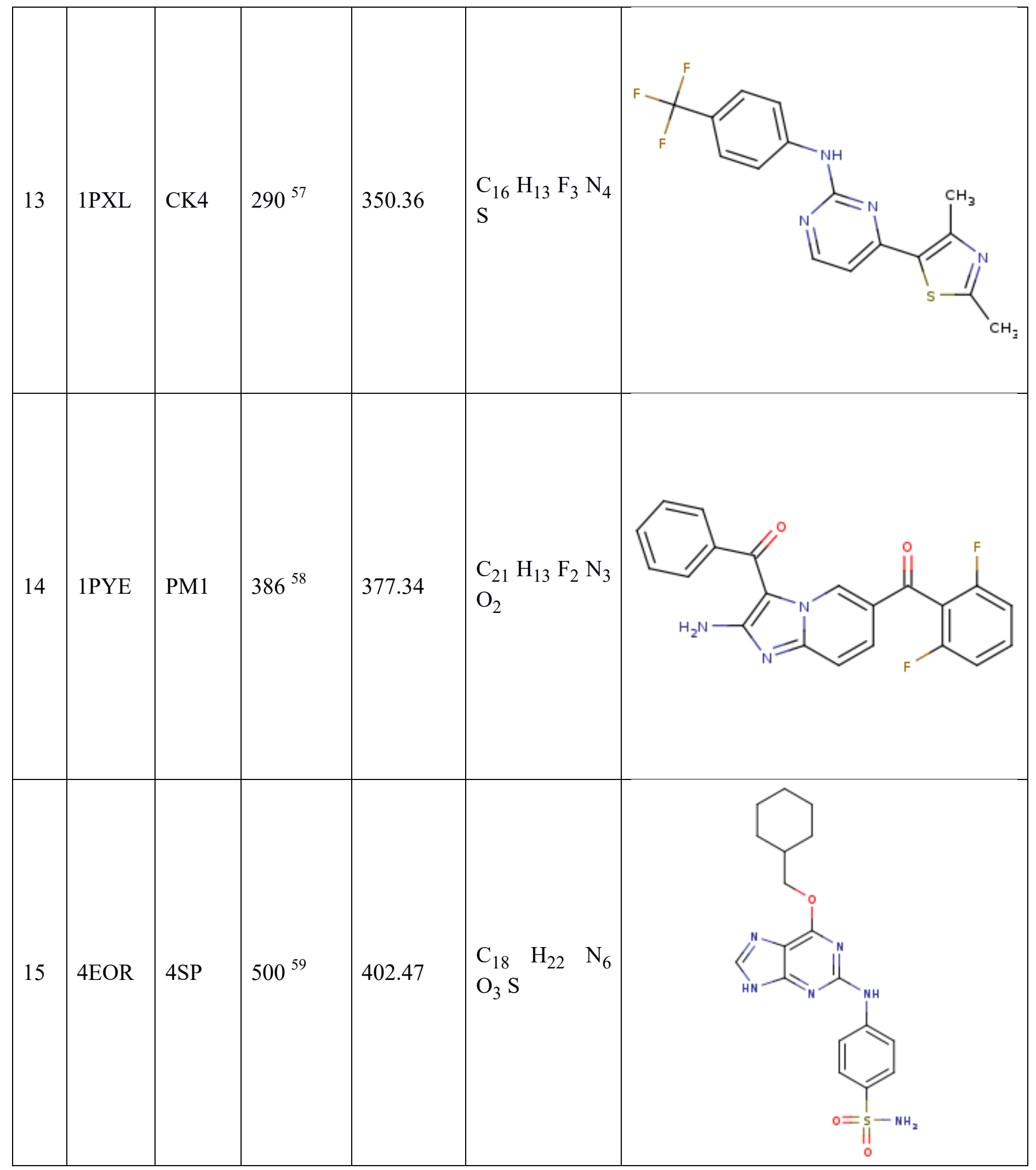




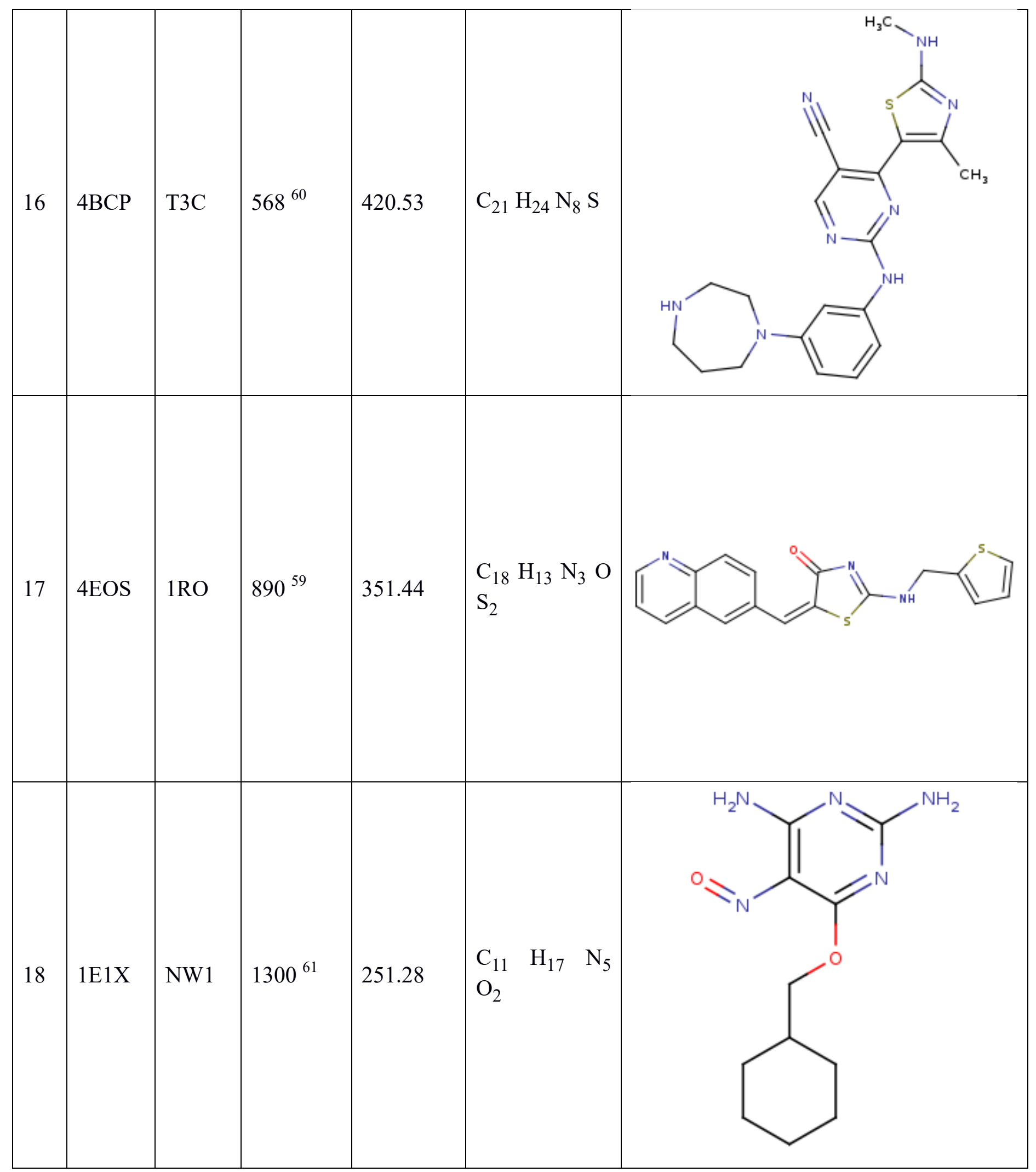




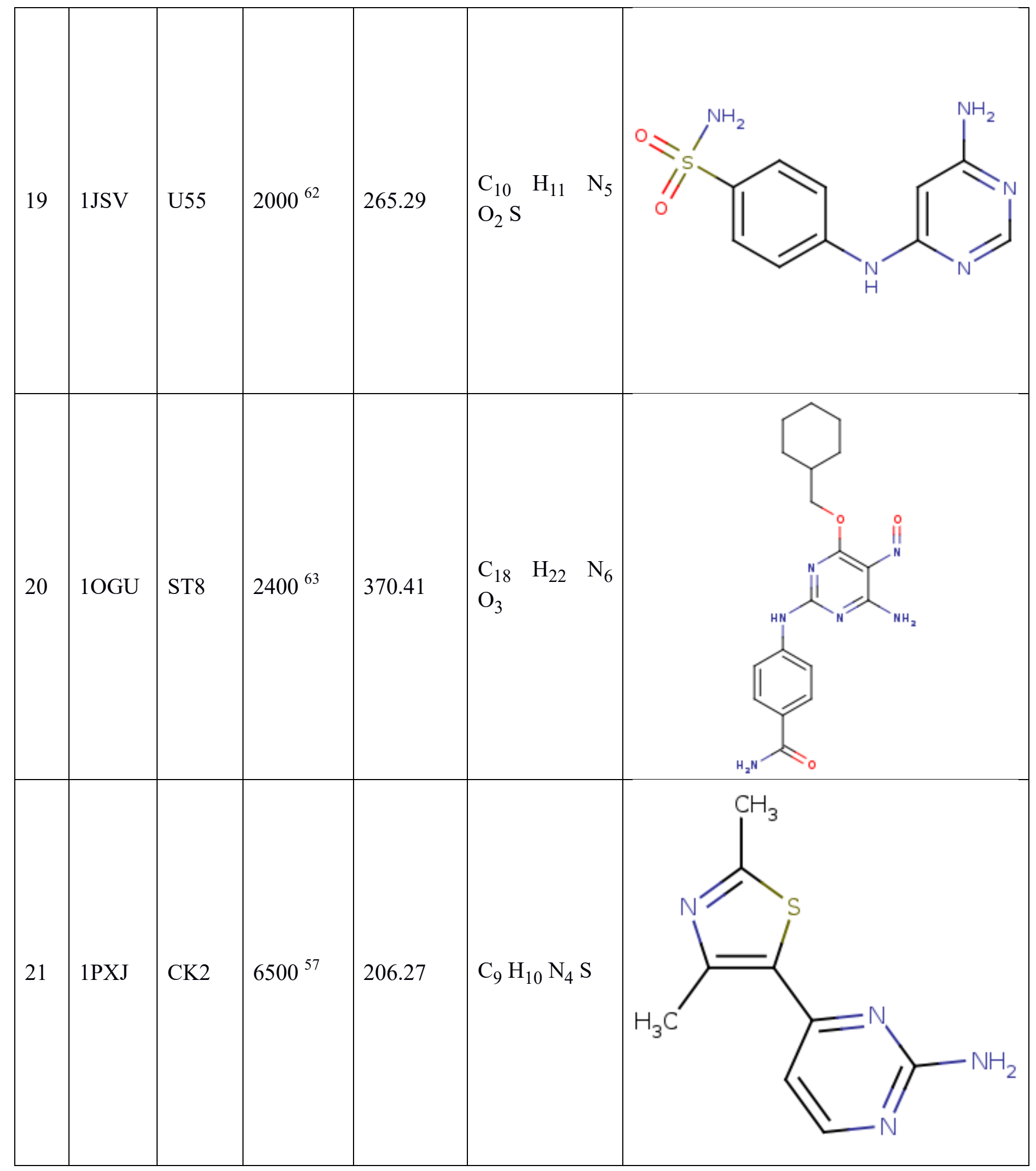




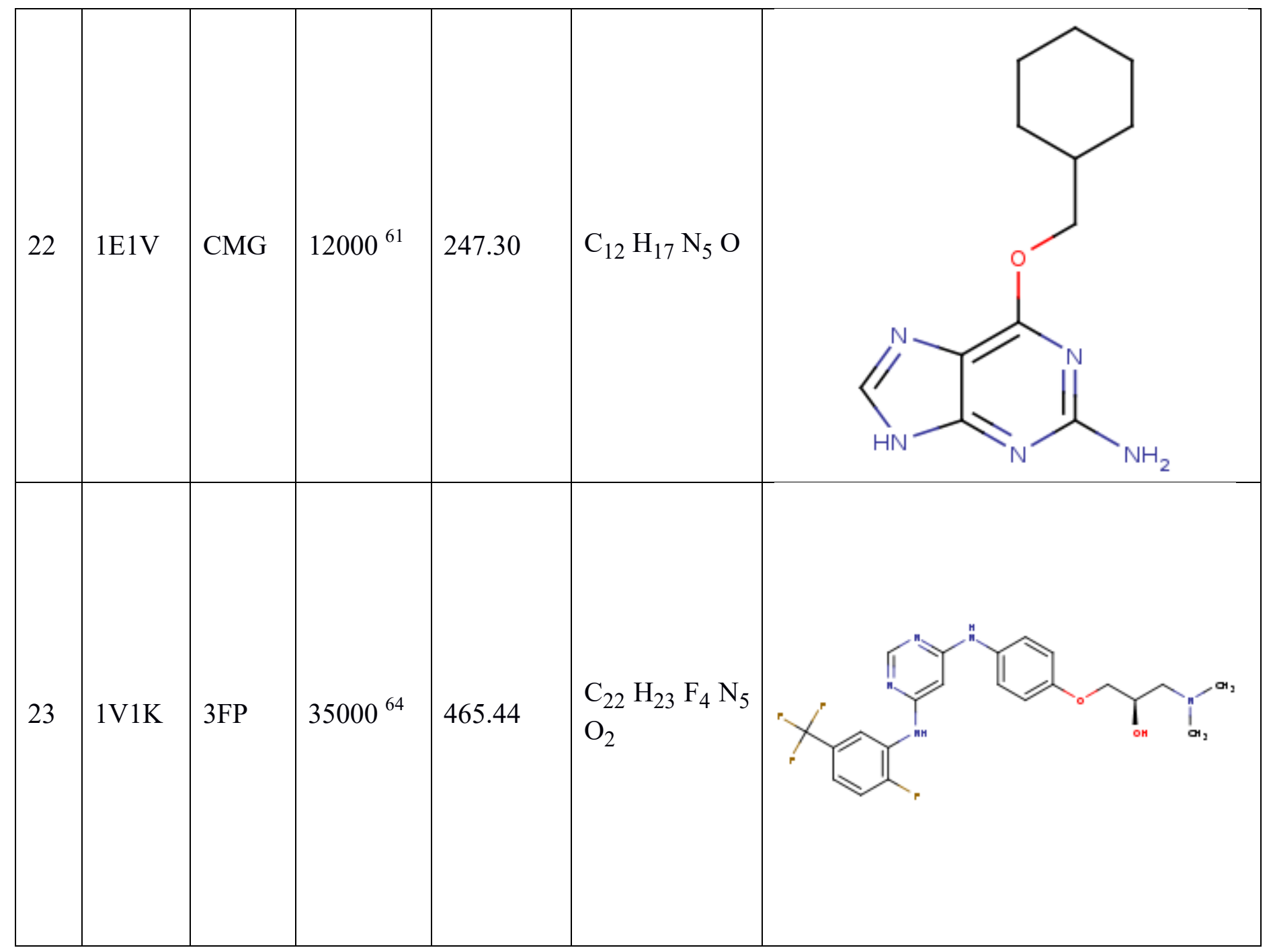

Table S6 - Scale factor $\beta$ for thrombin complexes.

\begin{tabular}{|l|l|l|c|}
\hline No. & Ligand PDB ID & Complex PDB ID & $\beta$ \\
\hline 1 & 170 & 1SL3 & 0.50 \\
\hline 2 & IH2 & 1C4V & 0.43 \\
\hline 3 & IH1 & 1C4U & 0.43 \\
\hline 4 & $33 \mathrm{U}$ & 2ZO3 & 0.43 \\
\hline 5 & 177 & 1TA6 & 0.43 \\
\hline 6 & T76 & 1NT1 & 0.43 \\
\hline 7 & BM9 & 1BMN & 0.37 \\
\hline 8 & MID & 1ETS & 0.43 \\
\hline 9 & 894 & 2JH6 & 0.43 \\
\hline 10 & $23 \mathrm{U}$ & 3DHK & 0.43 \\
\hline 11 & $64 \mathrm{U}$ & 3DUX & 0.43 \\
\hline 12 & $22 \mathrm{U}$ & 2ZC9 & 0.43 \\
\hline 13 & $29 \mathrm{U}$ & 2ZGX & 0.43 \\
\hline 14 & 895 & 2JH5 & 0.43 \\
\hline 15 & GR3 & 1AWH & 0.33 \\
\hline 16 & $00 \mathrm{R}$ & 1D6W & 0.43 \\
\hline 17 & B01 & 3SHC & 0.43 \\
\hline
\end{tabular}




\begin{tabular}{|l|l|l|l|}
\hline 18 & P97 & 3SHA & 0.43 \\
\hline 19 & $19 \mathrm{U}$ & 2ZFP & 0.43 \\
\hline 20 & $91 \mathrm{U}$ & 3F68 & 0.43 \\
\hline 21 & M18 & 3EGK & 0.43 \\
\hline 22 & $99 \mathrm{P}$ & 3P17 & 0.43 \\
\hline 23 & P05 & 3SV2 & 0.43 \\
\hline
\end{tabular}


Table S7 - Scale factor $\beta$ for factor Xa complexes

\begin{tabular}{|l|l|l|l|}
\hline No. & Ligand PDB ID & PDB ID & $\beta$ \\
\hline 1 & 993 & 2P3T & 0.43 \\
\hline 2 & XLD & 1MQ6 & 0.43 \\
\hline 3 & LG0 & 3CS7 & 0.43 \\
\hline 4 & 5QC & 2FZZ & 0.37 \\
\hline 5 & IIB & 2BQ6 & 0.43 \\
\hline 6 & GG2 & 2P16 & 0.43 \\
\hline 7 & Z34 & 1FJS & 0.33 \\
\hline 8 & 4QC & 2G00 & 0.43 \\
\hline 9 & IK8 & 1Z6E & 0.43 \\
\hline 10 & RIV & 2P95 & 0.43 \\
\hline 11 & ME5 & 2W26 & 0.43 \\
\hline 12 & XLC & 1MQ5 & 0.43 \\
\hline 13 & GSJ & 2J4I & 0.43 \\
\hline 14 & ME1 & 2P93 & 0.43 \\
\hline 15 & IIA & 2BOH & 0.43 \\
\hline 16 & IIE & 2BQW & 0.43 \\
\hline 17 & GSX & 2J95 & 0.43 \\
\hline 18 & GS6 & 2J34 & 0.43 \\
\hline 19 & DX9 & 1FAX & 0.37 \\
\hline 20 & GS5 & 2J38 & 0.43 \\
\hline 21 & IID & 2BQ7 & 0.43 \\
\hline 22 & 894 & 2UWP & 0.43 \\
\hline 23 & G15 & 2J94 & 0.43 \\
\hline & & & \\
\hline
\end{tabular}

Table S8 - Scale factor $\beta$ for beta-lactamase complexes

\begin{tabular}{|l|l|l|l|}
\hline No. & Ligand PDB ID & PDB ID & $\beta$ \\
\hline 1 & HTC & 1XGJ & 0.33 \\
\hline 2 & 23C & 2R9W & 0.33 \\
\hline 3 & NST & 1XGI & 0.37 \\
\hline 4 & WH6 & 2R9X & 0.33 \\
\hline 5 & $18 \mathrm{U}$ & 4JXS & 0.33 \\
\hline 6 & 1MW & 4JXW & 0.33 \\
\hline 7 & STC & 1L2S & 0.37 \\
\hline 8 & 1MU & 4JXV & 0.33 \\
\hline 9 & DK2 & 2PU2 & 0.33 \\
\hline 10 & $4 \mathrm{~A} 1$ & 4KZ4 & 0.37 \\
\hline 11 & NZ9 & 4KZA & 0.37 \\
\hline 12 & ZB6 & 4KZ6 & 0.37 \\
\hline 13 & G14 & 3GRJ & 0.37 \\
\hline 14 & $1 \mathrm{U} 1$ & 4KZ3 & 0.43 \\
\hline 15 & $1 \mathrm{U} 6$ & 4KZ8 & 0.37 \\
\hline 16 & GF1 & 3GSG & 0.37 \\
\hline
\end{tabular}




\begin{tabular}{|l|l|l|l|}
\hline 17 & GF4 & 3GR2 & 0.33 \\
\hline 18 & 3GV & 3GVB & 0.37 \\
\hline 19 & 1U5 & 4KZ7 & 0.37 \\
\hline 20 & F12 & 2HDU & 0.37 \\
\hline 21 & GV9 & 3GV9 & 0.37 \\
\hline 22 & 1U3 & 4KZ5 & 0.37 \\
\hline 23 & 4A3 & 2HDR & 0.33 \\
\hline
\end{tabular}

Table S9 - Scale factor $\beta$ for HIV-1 complexes

\begin{tabular}{|l|l|l|l|}
\hline No. & Ligand PDB ID & PDB ID & $\beta$ \\
\hline 1 & A79 & 1HVK & 0.33 \\
\hline 2 & A76 & 1HVL & 0.33 \\
\hline 3 & BEB & 1EBY & 0.33 \\
\hline 4 & MK1 & 1C6Y & 0.33 \\
\hline 5 & MK1 & 2BPX & 0.33 \\
\hline 6 & MS3 & 1EC3 & 0.33 \\
\hline 7 & BEE & 1EC1 & 0.33 \\
\hline 8 & 1 AH & 2CEJ & 0.33 \\
\hline 9 & HI & 2UXZ & 0.33 \\
\hline 10 & MSC & 1D4J & 0.33 \\
\hline 11 & 4 AH & 2CEN & 0.33 \\
\hline 12 & AHF & 1G35 & 0.33 \\
\hline 13 & A1A & 2BQV & 0.33 \\
\hline 14 & IM1 & 1SBG & 0.37 \\
\hline 15 & $1 \mathrm{IN}$ & 2BPV & 0.33 \\
\hline 16 & $3 \mathrm{IN}$ & 2BPY & 0.33 \\
\hline 17 & U02 & 2UPJ & 0.37 \\
\hline 18 & HV1 & 2UY0 & 0.33 \\
\hline 19 & G4G & 2PWR & 0.43 \\
\hline 20 & G3G & 2PWC & 0.43 \\
\hline 21 & GAN & 1HBV & 0.43 \\
\hline 22 & LJG & 3BGB & 0.43 \\
\hline 23 & LJH & 3BGC & 0.43 \\
\hline & & & \\
\hline
\end{tabular}

Table S10 - Scale factor $\beta$ for mcl-1 complexes

\begin{tabular}{|l|l|l|l|}
\hline No. & Ligand PDB ID & PDB ID & $\beta$ \\
\hline 1 & KJP & 6NE5 & 0.37 \\
\hline 2 & N0S & 6OQC & 0.37 \\
\hline 3 & N0J & 6OQB & 0.37 \\
\hline 4 & Q51 & 6UDV & 0.37 \\
\hline 5 & Q4D & 6UD2 & 0.37 \\
\hline 6 & Q4S & 6UDI & 0.37 \\
\hline 7 & Q4V & 6UDT & 0.33 \\
\hline 8 & N0M & 6OQD & 0.37 \\
\hline 9 & $5 X 3$ & 5FDR & 0.37 \\
\hline
\end{tabular}




\begin{tabular}{|l|l|l|l|}
\hline 10 & Q4Y & 6UDU & 0.33 \\
\hline 11 & ECM & 6BW8 & 0.37 \\
\hline 12 & CN7 & 6B4U & 0.37 \\
\hline 13 & ECY & 6BW2 & 0.37 \\
\hline 14 & 6AL & 5IEZ & 0.37 \\
\hline 15 & JL5 & 6QYP & 0.33 \\
\hline 16 & JL8 & 6QZ8 & 0.33 \\
\hline 17 & $19 \mathrm{H}$ & 4HW2 & 0.37 \\
\hline 18 & $5 \mathrm{WL}$ & 5FC4 & 0.43 \\
\hline 19 & 4M6 & 4ZBI & 0.37 \\
\hline 20 & $19 \mathrm{G}$ & 4HW3 & 0.37 \\
\hline 21 & CJY & 6B4L & 0.37 \\
\hline 22 & $5 \mathrm{X} 2$ & 5FDO & 0.43 \\
\hline 23 & JLK & 6QZB & 0.37 \\
\hline
\end{tabular}

Table S11 - Scale factor $\beta$ for CDK-2 complexes

\begin{tabular}{|l|l|l|l|}
\hline No. & Ligand PDB ID & PDB ID & $\beta$ \\
\hline 1 & LIA & 2FVD & 0.43 \\
\hline 2 & T3E & 4BCK & 0.43 \\
\hline 3 & T9N & 4BCN & 0.37 \\
\hline 4 & SU9 & 1PF8 & 0.43 \\
\hline 5 & $56 \mathrm{H}$ & 5D1J & 0.43 \\
\hline 6 & CK5 & 1PXM & 0.37 \\
\hline 7 & CK6 & 1PXN & 0.37 \\
\hline 8 & T7Z & 4BCM & 0.43 \\
\hline 9 & T6Q & 4BCO & 0.43 \\
\hline 10 & TJF & 4BCQ & 0.43 \\
\hline 11 & $7 \mathrm{YG}$ & 4ACM & 0.43 \\
\hline 12 & CK8 & 1PXP & 0.43 \\
\hline 13 & CK4 & 1PXL & 0.43 \\
\hline 14 & PM1 & 1PYE & 0.43 \\
\hline 15 & 4 SP & 4EOR & 0.43 \\
\hline 16 & T3C & 4BCP & 0.43 \\
\hline 17 & 1 RO & 4EOS & 0.43 \\
\hline 18 & NW1 & 1E1X & 0.43 \\
\hline 19 & U55 & 1JSV & 0.43 \\
\hline 20 & ST8 & 1OGU & 0.43 \\
\hline 21 & CK2 & 1PXJ & 0.43 \\
\hline 22 & CMG & 1E1V & 0.43 \\
\hline 23 & $3 F P$ & 1V1K & 0.37 \\
\hline & & \\
\hline
\end{tabular}

Table S12. SMD results obtained for 23 factor Xa complexes. $\Delta G_{\text {neq }}^{J a r}, \Delta G_{\text {unbind }}^{\ddagger}$, and $\Delta G_{\text {bind }}^{\ddagger}$ were obtained using the Jarzynski's equality.

\begin{tabular}{|l|l|l|l|l|l|}
\hline No. & Ligand & Complex & $\Delta G_{\text {exp }}$ & $F_{\max }$ & $W_{\text {pull }}$ \\
\hline
\end{tabular}




\begin{tabular}{|c|c|c|c|c|c|c|c|c|c|}
\hline & PDB ID & PDB ID & $(\mathrm{kcal} / \mathrm{mol})$ & $(\mathrm{pN})$ & $(\mathrm{kcal} / \mathrm{mol})$ & $(\mathrm{kcal} / \mathrm{mol})$ & (ps) & $(\mathrm{kcal} / \mathrm{mol})$ & $(\mathrm{kcal} / \mathrm{mol})$ \\
\hline 1 & 993 & $2 \mathrm{P} 3 \mathrm{~T}$ & -15.5 & $1057 \pm 38$ & $115 \pm 9$ & $-95.0 \pm 1.9$ & $231 \pm 12$ & $73.6 \pm 8.1$ & $188.7 \pm 14.0$ \\
\hline 2 & XLD & 1MQ6 & -15.3 & $1199 \pm 101$ & $140 \pm 17$ & $-90.5 \pm 1.9$ & $250 \pm 20$ & $101.4 \pm 18.2$ & $240.7 \pm 34.4$ \\
\hline 3 & LG0 & $3 \mathrm{CS} 7$ & -14.6 & $1108 \pm 86$ & $115 \pm 11$ & $-96.0 \pm 1.9$ & $232 \pm 18$ & $86.6 \pm 14.5$ & $200.7 \pm 24.4$ \\
\hline 4 & $5 \mathrm{QC}$ & $2 \mathrm{FZZ}$ & -14.4 & $1038 \pm 106$ & $117 \pm 14$ & $-83.4 \pm 1.9$ & $223 \pm 17$ & $71.9 \pm 18.4$ & $188.4 \pm 31.0$ \\
\hline 5 & IIB & 2BQ6 & -13.9 & $928 \pm 93$ & $97 \pm 12$ & $-76.3 \pm 1.9$ & $191 \pm 20$ & $62.0 \pm 12.0$ & $158.3 \pm 22.5$ \\
\hline 6 & GG2 & $2 \mathrm{P} 16$ & -13.8 & $1150 \pm 132$ & $126 \pm 19$ & $-84.0 \pm 1.9$ & $237 \pm 27$ & $95.5 \pm 20.7$ & $221.0 \pm 38.6$ \\
\hline 7 & $\mathrm{Z} 34$ & 1FJS & -13.7 & $1132 \pm 89$ & $124 \pm 14$ & $-87.8 \pm 1.9$ & $240 \pm 18$ & $86.4 \pm 16.6$ & $210.3 \pm 28.1$ \\
\hline 8 & $4 \mathrm{QC}$ & $2 \mathrm{G} 00$ & -13.4 & $1056 \pm 118$ & $119 \pm 18$ & $-92.1 \pm 1.8$ & $217 \pm 27$ & $79.0 \pm 17.6$ & $197.6 \pm 35.4$ \\
\hline 9 & IK8 & $1 \mathrm{Z6E}$ & -13.3 & $990 \pm 117$ & $107 \pm 14$ & $-88.8 \pm 1.7$ & $213 \pm 24$ & $67.3 \pm 19.0$ & $174.1 \pm 31.0$ \\
\hline 10 & RIV & $2 \mathrm{~W} 26$ & -12.9 & $1079 \pm 80$ & $124 \pm 16$ & $-80.2 \pm 1.9$ & $230 \pm 22$ & $78.9 \pm 13.2$ & $202.7 \pm 24.5$ \\
\hline 11 & ME5 & $2 \mathrm{P} 95$ & -12.8 & $864 \pm 31$ & $82 \pm 5$ & $-72.0 \pm 1.9$ & $188 \pm 7$ & $51.3 \pm 5.5$ & $132.5 \pm 6.8$ \\
\hline 12 & XLC & 1MQ5 & -12.4 & $911 \pm 80$ & $97 \pm 9$ & $-76.9 \pm 1.9$ & $197 \pm 19$ & $55.7 \pm 12.2$ & $152.2 \pm 18.7$ \\
\hline 13 & GSJ & $2 \mathrm{~J} 4 \mathrm{I}$ & -12.4 & $1028 \pm 133$ & $120 \pm 16$ & $-86.1 \pm 1.9$ & $227 \pm 26$ & $68.5 \pm 21.6$ & $187.8 \pm 37.3$ \\
\hline 14 & ME1 & $2 \mathrm{P} 93$ & -12.1 & $844 \pm 74$ & $80 \pm 6$ & $-70.6 \pm 1.7$ & $176 \pm 16$ & $50.9 \pm 9.1$ & $130.5 \pm 13.8$ \\
\hline 15 & IIA & $2 \mathrm{BOH}$ & -11.7 & $911 \pm 50$ & $91 \pm 5$ & $-77.9 \pm 1.9$ & $187 \pm 10$ & $59.7 \pm 7.4$ & $150.2 \pm 10.8$ \\
\hline 16 & IIE & 2BQW & -11.7 & $1067 \pm 112$ & $111 \pm 17$ & $-82.3 \pm 1.9$ & $217 \pm 22$ & $82.4 \pm 18.3$ & $192.8 \pm 35.6$ \\
\hline 17 & GSX & 2J95 & -11.5 & $816 \pm 56$ & $82 \pm 6$ & $-72.7 \pm 1.7$ & $172 \pm 14$ & $47.2 \pm 7.0$ & $129.1 \pm 12.0$ \\
\hline 18 & GS6 & $2 \mathrm{~J} 34$ & -10.7 & $782 \pm 68$ & $80 \pm 6$ & $-67.5 \pm 1.9$ & $166 \pm 11$ & $42.7 \pm 10.2$ & $122.0 \pm 14.9$ \\
\hline 19 & DX9 & $1 F A X$ & -10.1 & $868 \pm 77$ & $98 \pm 9$ & $-78.3 \pm 1.9$ & $193 \pm 18$ & $48.5 \pm 14.9$ & $145.9 \pm 18.3$ \\
\hline 20 & GS5 & $2 \mathrm{~J} 38$ & -10.0 & $767 \pm 66$ & $73 \pm 7$ & $-62.1 \pm 1.8$ & $168 \pm 12$ & $39.2 \pm 9.6$ & $112.0 \pm 15.5$ \\
\hline 21 & IID & 2BQ7 & -9.7 & $960 \pm 125$ & $92 \pm 16$ & $-66.2 \pm 1.9$ & $194 \pm 28$ & $67.7 \pm 18.1$ & $159.2 \pm 34.0$ \\
\hline 22 & 894 & 2UWP & -9.4 & $822 \pm 74$ & $88 \pm 9$ & $-70.3 \pm 1.8$ & $182 \pm 20$ & $43.4 \pm 13.4$ & $130.7 \pm 16.6$ \\
\hline 23 & G15 & $2 \mathrm{~J} 94$ & -8.6 & $695 \pm 51$ & $71 \pm 5$ & $-62.8 \pm 1.9$ & $174 \pm 12$ & $27.3 \pm 7.5$ & $98.2 \pm 10.7$ \\
\hline
\end{tabular}

Table S13. SMD results obtained for 23 HIV-1 complexes. $\Delta G_{\text {neq }}^{J a r}, \Delta G_{\text {unbind }}^{\ddagger}$, and $\Delta G_{\text {bind }}^{\ddagger}$ were obtained using the Jarzynski's equality.

\begin{tabular}{|c|c|c|c|c|c|c|c|c|c|}
\hline No. & $\begin{array}{c}\text { Ligand } \\
\text { PDB ID }\end{array}$ & $\begin{array}{c}\text { Complex } \\
\text { PDB ID }\end{array}$ & $\begin{array}{c}\Delta G_{\text {exp }} \\
(\mathrm{kcal} / \mathrm{mol})\end{array}$ & $\begin{array}{c}F_{\max } \\
(\mathrm{pN})\end{array}$ & $\begin{array}{c}W_{\text {pull }} \\
(\mathrm{kcal} / \mathrm{mol})\end{array}$ & $\begin{array}{c}\Delta G_{\text {neq }}^{\text {Jar }} \\
(\mathrm{kcal} / \mathrm{mol})\end{array}$ & $\begin{array}{c}t_{\text {max }} \\
(\mathrm{ps})\end{array}$ & $\begin{array}{c}\Delta G_{\text {unbind }}^{\ddagger} \\
(\mathrm{kcal} / \mathrm{mol})\end{array}$ & $\begin{array}{c}\Delta G_{\text {bind }}^{\ddagger} \\
(\mathrm{kcal} / \mathrm{mol})\end{array}$ \\
\hline 1 & A79 & $1 \mathrm{HVK}$ & -15.04 & $1984 \pm 229$ & $359 \pm 58$ & $-280.2 \pm 1.9$ & $423 \pm 44$ & $272.7 \pm 69.7$ & $631.6 \pm 127.3$ \\
\hline 2 & A76 & 1HVL & -13.66 & $2065 \pm 255$ & $392 \pm 71$ & $-260.6 \pm 1.9$ & $446 \pm 50$ & $293.0 \pm 80.6$ & $684.9 \pm 151.6$ \\
\hline 3 & BEB & 1EBY & -13.31 & $1782 \pm 221$ & $321 \pm 52$ & $-236.7 \pm 1.9$ & $397 \pm 46$ & $214.2 \pm 62.0$ & $534.6 \pm 113.8$ \\
\hline 4 & MK1 & 1 C6Y & -13.05 & $1620 \pm 248$ & $281 \pm 51$ & $-183.4 \pm 2.0$ & $366 \pm 45$ & $174.4 \pm 63.1$ & $455.0 \pm 114.0$ \\
\hline 5 & MK1 & 2BPX & -12.9 & $1989 \pm 240$ & $386 \pm 74$ & $-250.3 \pm 2.0$ & $441 \pm 58$ & $259.4 \pm 71.1$ & $645.3 \pm 144.8$ \\
\hline 6 & MS3 & 1EC3 & -12.4 & $2244 \pm 325$ & $444 \pm 102$ & $-247.2 \pm 2.0$ & $470 \pm 67$ & $360.8 \pm 106.0$ & $804.1 \pm 208.2$ \\
\hline 7 & BEE & $1 \mathrm{EC1}$ & -12.24 & $1695 \pm 217$ & $302 \pm 51$ & $-206.1 \pm 1.9$ & $389 \pm 49$ & $183.9 \pm 57.6$ & $485.2 \pm 108.8$ \\
\hline 8 & 1 AH & 2CEJ & -11.83 & $2011 \pm 288$ & $361 \pm 78$ & $-208.2 \pm 2.0$ & $432 \pm 61$ & $280.1 \pm 83.2$ & $640.6 \pm 161.7$ \\
\hline 9 & HI1 & 2UXZ & -11.64 & $1939 \pm 174$ & $337 \pm 44$ & $-243.0 \pm 2.0$ & $415 \pm 35$ & $258.0 \pm 50.8$ & $595.0 \pm 95.3$ \\
\hline
\end{tabular}




\begin{tabular}{|c|c|c|c|c|c|c|c|c|c|}
\hline 10 & MSC & 1D4J & -11.47 & $2082 \pm 237$ & $382 \pm 66$ & $-250.2 \pm 2.0$ & $442 \pm 49$ & $301.7 \pm 66.8$ & $683.6 \pm 132.4$ \\
\hline 11 & 4AH & 2CEN & -11.39 & $1838 \pm 252$ & $323 \pm 63$ & $-195.1 \pm 2.0$ & $401 \pm 56$ & $230.9 \pm 61.7$ & $554.0 \pm 124.5$ \\
\hline 12 & AHF & $1 \mathrm{G} 35$ & -11.17 & $2090 \pm 245$ & $382 \pm 72$ & $-214.1 \pm 2.0$ & $451 \pm 48$ & $300.9 \pm 74.2$ & $682.0 \pm 146.0$ \\
\hline 13 & A1A & 2 BQV & -11.04 & $2037 \pm 233$ & $362 \pm 61$ & $-239.2 \pm 2.0$ & $433 \pm 48$ & $289.2 \pm 69.0$ & $650.8 \pm 130.2$ \\
\hline 14 & IM1 & $1 \mathrm{SBG}$ & -10.63 & $1485 \pm 198$ & $242 \pm 34$ & $-168.7 \pm 2.0$ & $325 \pm 42$ & $152.0 \pm 41.2$ & $393.6 \pm 75.1$ \\
\hline 15 & $1 \mathrm{IN}$ & 2BPV & -10.53 & $1713 \pm 274$ & $285 \pm 64$ & $-188.3 \pm 2.0$ & $373 \pm 55$ & $203.2 \pm 67.8$ & $487.9 \pm 132.0$ \\
\hline 16 & 3IN & 2BPY & -10.16 & $1532 \pm 203$ & $253 \pm 37$ & $-163.9 \pm 2.0$ & $337 \pm 44$ & $160.8 \pm 42.2$ & $413.4 \pm 79.4$ \\
\hline 17 & U02 & 2UPJ & -10.14 & $1394 \pm 221$ & $212 \pm 44$ & $-127.2 \pm 2.0$ & $322 \pm 52$ & $126.0 \pm 41.3$ & $337.8 \pm 85.4$ \\
\hline 18 & HV1 & 2UY0 & -9.5 & $1942 \pm 232$ & $344 \pm 54$ & $-231.2 \pm 1.9$ & $418 \pm 46$ & $260.4 \pm 66.1$ & $603.7 \pm 120.4$ \\
\hline 19 & G4G & 2PWR & -9.04 & $1613 \pm 262$ & $253 \pm 54$ & $-146.2 \pm 2.0$ & $348 \pm 54$ & $182.0 \pm 60.1$ & $434.5 \pm 113.8$ \\
\hline 20 & G3G & 2PWC & -9.02 & $1425 \pm 188$ & $213 \pm 38$ & $-134.3 \pm 2.0$ & $310 \pm 44$ & $143.6 \pm 41.4$ & $356.1 \pm 79.6$ \\
\hline 21 & GAN & 1HBV & -8.74 & $1712 \pm 328$ & $291 \pm 77$ & $-125.7 \pm 2.0$ & $372 \pm 71$ & $209.4 \pm 77.6$ & $500.0 \pm 154.3$ \\
\hline 22 & LJG & 3BGB & -8.3 & $1354 \pm 200$ & $206 \pm 32$ & $-146.3 \pm 2.0$ & $290 \pm 45$ & $130.2 \pm 38.6$ & $335.6 \pm 70.3$ \\
\hline 23 & LJH & 3BGC & -6.89 & $1300 \pm 211$ & $207 \pm 35$ & $-130.0 \pm 1.9$ & $294 \pm 43$ & $113.5 \pm 37.5$ & $319.8 \pm 72.8$ \\
\hline
\end{tabular}

Table S14. SMD results obtained for $23 \mathrm{MCL}-1$ complexes. $\Delta G_{n e q}^{J a r}, \Delta G_{\text {unbind }}^{\ddagger}$, and $\Delta G_{\text {bind }}^{\ddagger}$ were obtained using the Jarzynski's equality.

\begin{tabular}{|c|c|c|c|c|c|c|c|c|c|}
\hline No. & $\begin{array}{l}\text { Ligand } \\
\text { PDB ID }\end{array}$ & $\begin{array}{l}\text { Complex } \\
\text { PDB ID }\end{array}$ & $\begin{array}{c}\Delta G_{\text {exp }} \\
(\mathrm{kcal} / \mathrm{mol})\end{array}$ & $\begin{array}{l}F_{\max } \\
(\mathrm{pN}) \\
\end{array}$ & $\begin{array}{c}\begin{array}{c}W_{\text {pull }} \\
(\mathrm{kcal} / \mathrm{mol})\end{array}\end{array}$ & $\begin{array}{c}\Delta G_{\text {neq }}^{J a r} \\
(\mathrm{kcal} / \mathrm{mol})\end{array}$ & $\begin{array}{r}t_{\max } \\
(\mathrm{ps}) \\
\end{array}$ & $\begin{array}{c}\Delta G_{\text {unbind }}^{\ddagger} \\
(\mathrm{kcal} / \mathrm{mol})\end{array}$ & $\begin{array}{c}\Delta G_{b i n d}^{\ddagger} \\
(\mathrm{kcal} / \mathrm{mol})\end{array}$ \\
\hline 1 & KJP & 6NE5 & -14.44 & $1088 \pm 111$ & $120 \pm 18$ & $-81.7 \pm 1.9$ & $246 \pm 24$ & $77.7 \pm 16.6$ & $197.2 \pm 34.3$ \\
\hline 2 & NOS & $6 \mathrm{OQC}$ & -14.27 & $1239 \pm 147$ & $135 \pm 26$ & $-84.8 \pm 2.0$ & $257 \pm 32$ & $111.5 \pm 25.4$ & $246.6 \pm 51.6$ \\
\hline 3 & NOJ & $6 \mathrm{OQB}$ & -14.14 & $1135 \pm 100$ & $119 \pm 15$ & $-77.8 \pm 2.0$ & $241 \pm 21$ & $90.6 \pm 17.6$ & $209.4 \pm 32.5$ \\
\hline 4 & Q51 & 6UDV & -14.13 & $1126 \pm 106$ & $118 \pm 16$ & $-76.0 \pm 1.9$ & $243 \pm 22$ & $88.0 \pm 16.8$ & $205.7 \pm 32.5$ \\
\hline 5 & Q4D & 6UD2 & -14.01 & $1397 \pm 155$ & $180 \pm 33$ & $-79.9 \pm 2.0$ & $304 \pm 35$ & $130.5 \pm 27.5$ & $309.7 \pm 60.4$ \\
\hline 6 & Q4S & 6UDI & -13.73 & $1408 \pm 134$ & $171 \pm 28$ & $-88.0 \pm 2.0$ & $299 \pm 29$ & $138.8 \pm 26.3$ & $309.3 \pm 53.8$ \\
\hline 7 & $\mathrm{Q} 4 \mathrm{~V}$ & 6UDT & & $1093 \pm 214$ & $116 \pm 33$ & $-61.8 \pm 2.0$ & $234 \pm 45$ & $86.7 \pm 32.5$ & $202.9 \pm 65.3$ \\
\hline 8 & NOM & 6OQD & -13.53 & $999 \pm 117$ & $104 \pm 16$ & $-68.4 \pm 2.0$ & $214 \pm 26$ & $69.8 \pm 17.4$ & $173.4 \pm 33.6$ \\
\hline 9 & $5 \times 3$ & 5FDR & -12.9 & $756 \pm 90$ & $73 \pm 8$ & $-57.4 \pm 1.8$ & $165 \pm 19$ & $40.3 \pm 9.6$ & $112.6 \pm 17.6$ \\
\hline 10 & Q4Y & 6UDU & -12.77 & $1452 \pm 152$ & $174 \pm 31$ & $-95.1 \pm 2.0$ & $302 \pm 31$ & $151.5 \pm 31.3$ & $325.3 \pm 62.8$ \\
\hline 11 & ECM & $6 \mathrm{BW} 8$ & -12.61 & $1108 \pm 109$ & $121 \pm 20$ & $-91.2 \pm 1.8$ & $237 \pm 24$ & $85.3 \pm 16.3$ & $205.7 \pm 35.9$ \\
\hline 12 & CN7 & $6 \mathrm{~B} 4 \mathrm{U}$ & -11.04 & $728 \pm 97$ & $67 \pm 8$ & $-52.1 \pm 1.9$ & $158 \pm 21$ & $38.8 \pm 11.0$ & $105.7 \pm 18.8$ \\
\hline 13 & ECY & 6BW2 & -10.54 & $761 \pm 142$ & $77 \pm 13$ & $-46.6 \pm 1.8$ & $164 \pm 30$ & $42.1 \pm 14.5$ & $118.8 \pm 27.5$ \\
\hline 14 & $6 \mathrm{AL}$ & 5IEZ & -10.48 & $731 \pm 73$ & $74 \pm 7$ & $-58.4 \pm 2.0$ & $163 \pm 16$ & $37.6 \pm 8.0$ & $110.8 \pm 15.2$ \\
\hline 15 & JL5 & 6QYP & -10.07 & $712 \pm 77$ & $62 \pm 8$ & $-40.3 \pm 2.0$ & $152 \pm 16$ & $36.8 \pm 7.9$ & $98.1 \pm 16.5$ \\
\hline 16 & JL8 & 6QZ8 & -10.01 & $760 \pm 87$ & $62 \pm 8$ & $-45.3 \pm 1.9$ & $158 \pm 20$ & $43.3 \pm 9.5$ & $105.4 \pm 18.1$ \\
\hline 17 & $19 \mathrm{H}$ & 4HW2 & & $833 \pm 94$ & $78 \pm 12$ & $-46.5 \pm 2.0$ & $181 \pm 21$ & $49.3 \pm 10.5$ & $127.5 \pm 22.2$ \\
\hline 18 & $5 \mathrm{WL}$ & & & $537 \pm 58$ & $50 \pm 7$ & $-34.0 \pm 2.0$ & $125 \pm 19$ & $21.3 \pm 4.8$ & $71.0 \pm 12.1$ \\
\hline 19 & $4 \mathrm{M} 6$ & 4ZBI & -8.93 & $800 \pm 107$ & $75 \pm 10$ & $-55.1 \pm 1.9$ & $173 \pm 22$ & $46.5 \pm 11.5$ & $121.1 \pm 21.2$ \\
\hline 20 & $19 \mathrm{G}$ & 4HW3 & -8.91 & $815 \pm 124$ & $76 \pm 13$ & $-49.2 \pm 1.9$ & $178 \pm 28$ & $47.5 \pm 13.9$ & $123.3 \pm 27.2$ \\
\hline 21 & CJY & 6B4L & -8.88 & $773 \pm 93$ & $67 \pm 11$ & $-43.8 \pm 2.0$ & $165 \pm 24$ & $43.2 \pm 9.0$ & $110.0 \pm 20.2$ \\
\hline 22 & $5 \times 2$ & $5 \mathrm{FDO}$ & -8.84 & $798 \pm 110$ & $76 \pm 10$ & $-57.2 \pm 1.9$ & $171 \pm 25$ & $45.3 \pm 12.4$ & $120.6 \pm 22.0$ \\
\hline 23 & JLK & 6QZB & -7.71 & $613 \pm 82$ & $48 \pm 7$ & $-26.9 \pm 2.0$ & $131 \pm 18$ & $27.8 \pm 7.5$ & $75.0 \pm 14.6$ \\
\hline
\end{tabular}

Table S15. SMD results obtained for 23 CDK-2 complexes. $\Delta G_{\text {neq }}^{J a r}, \Delta G_{\text {unbind }}^{\ddagger}$, and $\Delta G_{\text {bind }}^{\ddagger}$ were obtained using the Jarzynski's equality. 


\begin{tabular}{|c|c|c|c|c|c|c|c|c|c|}
\hline No. & $\begin{array}{l}\text { Ligand } \\
\text { PDB ID }\end{array}$ & $\begin{array}{l}\text { Complex } \\
\text { PDB ID }\end{array}$ & $\begin{array}{c}\Delta G_{\text {exp }} \\
(\mathrm{kcal} / \mathrm{mol})\end{array}$ & $\begin{array}{l}F_{\max } \\
(\mathrm{pN})\end{array}$ & $\begin{array}{c}\begin{array}{c}W_{\text {pull }} \\
(\mathrm{kcal} / \mathrm{mol})\end{array}\end{array}$ & $\begin{array}{c}\Delta G_{\text {neq }}^{J a r} \\
(\mathrm{kcal} / \mathrm{mol})\end{array}$ & $\begin{array}{c}t_{\max } \\
(\mathrm{ps})\end{array}$ & $\begin{array}{c}\Delta G_{\text {unbind }}^{\ddagger} \\
(\mathrm{kcal} / \mathrm{mol})\end{array}$ & $\begin{array}{c}\Delta G_{b i n d}^{\ddagger} \\
(\mathrm{kcal} / \mathrm{mol})\end{array}$ \\
\hline 1 & LIA & $2 \mathrm{FVD}$ & -11.7 & $1602 \pm 230$ & $230 \pm 43$ & $-153.0 \pm 1.9$ & $334 \pm 44$ & $194.0 \pm 48.4$ & $423.9 \pm 107.7$ \\
\hline 2 & T3E & $4 \mathrm{BCK}$ & -11.53 & $1346 \pm 207$ & $200 \pm 30$ & $-159.8 \pm 1.9$ & $296 \pm 42$ & $149.9 \pm 34.0$ & $312.7 \pm 70.2$ \\
\hline 3 & T9N & $4 \mathrm{BCN}$ & -10.87 & $1492 \pm 124$ & $226 \pm 18$ & $-154.6 \pm 1.9$ & $330 \pm 31$ & $151.7 \pm 27.6$ & $376.8 \pm 39.0$ \\
\hline 4 & SU9 & 1PF8 & -10.31 & $1285 \pm 114$ & $156 \pm 28$ & $-118.7 \pm 1.9$ & $281 \pm 26$ & $113.2 \pm 21.8$ & $264.6 \pm 42.1$ \\
\hline 5 & $56 \mathrm{H}$ & 5D1J & -10.18 & $1611 \pm 131$ & $219 \pm 27$ & $-158.7 \pm 1.9$ & $344 \pm 27$ & $173.5 \pm 22.7$ & $403.3 \pm 53.5$ \\
\hline 6 & CK5 & 1PXM & -9.91 & $999 \pm 191$ & $139 \pm 51$ & $-103.9 \pm 1.8$ & $220 \pm 41$ & $88.2 \pm 22.1$ & $226.9 \pm 52.4$ \\
\hline 7 & CK6 & $1 \mathrm{PXN}$ & -9.82 & $1630 \pm 236$ & $219 \pm 57$ & $-125.8 \pm 2.0$ & & $185.5 \pm 52.9$ & $409.8 \pm 99.6$ \\
\hline 8 & & & -9.48 & $1212 \pm 94$ & $191 \pm 25$ & $-145.5 \pm 1.8$ & $300 \pm$ & $91.2 \pm 18.1$ & $279.1 \pm 36.9$ \\
\hline 9 & T6Q & $\mathrm{CO}$ & -9.45 & $1394 \pm 143$ & $36 \pm 26$ & $-138.2 \pm 1.9$ & $303 \pm 27$ & $128.4 \pm 2$ & $324.8 \pm 59.1$ \\
\hline 10 & TJF & CQ & -9.38 & $1214 \pm 1$ & $3 \pm 29$ & $.0 \pm 1.9$ & & $101.7 \pm 27.6$ & $254.1 \pm 53.5$ \\
\hline 11 & $Y G$ & $4 \mathrm{ACM}$ & -9.17 & $1176 \pm 192$ & $178 \pm 28$ & $-120.5 \pm 1.9$ & $275 \pm 47$ & $92.7 \pm 25.5$ & $269.9 \pm 49.2$ \\
\hline 12 & CK8 & 1PXP & -9.14 & $1062 \pm 237$ & $140 \pm 27$ & $-97.0 \pm 1.9$ & $233 \pm 48$ & $96.5 \pm 35.2$ & $235.6 \pm 60.3$ \\
\hline 13 & CK4 & 1PXL & -8.97 & $1210 \pm 164$ & $144 \pm 24$ & $-102.1 \pm 1.9$ & $260 \pm 32$ & $105.4 \pm 29.7$ & $246.5 \pm 46.0$ \\
\hline 14 & PM1 & 1PYE & -8.8 & $990 \pm 111$ & $130 \pm 21$ & $-80.7 \pm 1.9$ & $220 \pm 32$ & $69.6 \pm 15.4$ & $198.6 \pm 30.8$ \\
\hline 15 & $4 \mathrm{SP}$ & 4EOR & -8.65 & $1372 \pm 124$ & $177 \pm 24$ & $-111.3 \pm 1.9$ & $305 \pm 26$ & $123.7 \pm 24.5$ & $300.3 \pm 47.2$ \\
\hline 16 & $\mathrm{~T} 3 \mathrm{C}$ & $4 \mathrm{BCP}$ & -8.57 & $1458 \pm 267$ & $192 \pm 34$ & $-122.5 \pm 1.9$ & $311 \pm 54$ & $134.5 \pm 40.0$ & $360.8 \pm 101.4$ \\
\hline 17 & $1 \mathrm{RO}$ & 4EOS & -8.3 & $1239 \pm 236$ & $168 \pm 44$ & $-95.7 \pm 1.9$ & $285 \pm 44$ & $110.9 \pm 43.1$ & $276.6 \pm 92.4$ \\
\hline 18 & & & & $1084 \pm 1$ & & $-73.9 \pm 1.9$ & & $82.1 \pm 2$ & $201.2 \pm 50.4$ \\
\hline 19 & & & & & & & & & $206.5 \pm 73.6$ \\
\hline 20 & ST8 & $1 \mathrm{OGU}$ & -7.71 & $1106 \pm 171$ & $123 \pm 27$ & $-78.6 \pm 1.9$ & $241 \pm 34$ & $87.7 \pm 27.6$ & $210.0 \pm 53.9$ \\
\hline 21 & CK2 & 1PXJ & -7.12 & $1207 \pm 161$ & $152 \pm 41$ & $-99.8 \pm 1.9$ & $260 \pm 34$ & $117.7 \pm 40.3$ & $261.2 \pm 70.4$ \\
\hline 22 & $\mathrm{CMG}$ & $1 \mathrm{E} 1 \mathrm{~V}$ & -6.75 & $926 \pm 191$ & $128 \pm 24$ & $-75.8 \pm 1.9$ & $235 \pm 52$ & $84.1 \pm 28.8$ & $208.1 \pm 45.8$ \\
\hline 23 & $3 \mathrm{FP}$ & $1 \mathrm{~V} 1 \mathrm{~K}$ & -6.12 & $946 \pm 136$ & $122 \pm 19$ & $-74.8 \pm 1.9$ & $227 \pm 30$ & $56.8 \pm 17.0$ & $174.9 \pm 35.0$ \\
\hline
\end{tabular}

Table S16 - Average polar and non-polar interaction energy in the bound and free state for 23 factor Xa complexes. Absolute binding free energy $\Delta G_{\mathrm{LIE}}$ was obtained for $\alpha=1$ (optimal value for a set of 23 compounds).

\begin{tabular}{|c|c|c|c|c|c|c|c|}
\hline No. & $\begin{array}{c}\text { Factor Xa } \\
\text { PDB ID }\end{array}$ & $\Delta G_{\text {exp }}$ & $\left\langle V^{\text {elec }}\right\rangle_{\text {bound }}$ & $\left\langle V^{\text {elec }}\right\rangle_{\text {free }}$ & $\left\langle V^{\text {vdw }}\right\rangle_{\text {bound }}$ & $\left\langle V^{\text {vdw }}\right\rangle_{\text {free }}$ & $\begin{array}{c}\Delta G_{\text {LIE }} \\
\alpha=1\end{array}$ \\
\hline 1 & $2 \mathrm{P} 3 \mathrm{~T}$ & -15.5 & $-43.1 \pm 4.7$ & $-37.1 \pm 3$ & $-118.9 \pm 5.8$ & $-37.1 \pm 3.1$ & $-84.4 \pm 12.3$ \\
\hline 2 & $1 \mathrm{MQ6}$ & -15.3 & $-41.7 \pm 3.1$ & $-31.3 \pm 2.6$ & $-135.4 \pm 5.1$ & $-35 \pm 2.9$ & $-104.8 \pm 10.4$ \\
\hline 3 & $3 \mathrm{CS} 7$ & -14.6 & $-20.9 \pm 2.1$ & $-16.4 \pm 1.6$ & $-121.8 \pm 5.5$ & $-41.4 \pm 2.5$ & $-82.3 \pm 9.6$ \\
\hline 4 & $2 \mathrm{FZZ}$ & -14.4 & $-38.6 \pm 3.8$ & $-29.6 \pm 2.6$ & $-121.2 \pm 6.8$ & $-40.7 \pm 3.4$ & $-83.8 \pm 12.6$ \\
\hline 5 & $2 \mathrm{BQ6}$ & -13.9 & $-25 \pm 2.9$ & $-20.1 \pm 2.3$ & $-119.6 \pm 3.9$ & $-38.6 \pm 2.6$ & $-83.1 \pm 8.7$ \\
\hline 6 & $2 \mathrm{P} 16$ & -13.8 & $-30.8 \pm 4.1$ & $-27.9 \pm 3$ & $-111 \pm 5.3$ & $-34.8 \pm 3.2$ & $-77.4 \pm 11.5$ \\
\hline 7 & $1 \mathrm{FJS}$ & -13.7 & $-50.4 \pm 5.5$ & $-49.1 \pm 3.4$ & $-104.5 \pm 4.5$ & $-27.7 \pm 4.2$ & $-77.2 \pm 11.6$ \\
\hline 8 & $2 \mathrm{G} 00$ & -13.4 & $-29.3 \pm 2.8$ & $-26.6 \pm 2.6$ & $-114.2 \pm 5.4$ & $-38.4 \pm 3.1$ & $-76.9 \pm 10.9$ \\
\hline 9 & $1 \mathrm{Z6E}$ & -13.3 & $-41.7 \pm 5$ & $-30.7 \pm 2.9$ & $-113.4 \pm 4.4$ & $-36 \pm 3.1$ & $-82.1 \pm 10.9$ \\
\hline 10 & $2 \mathrm{~W} 26$ & -12.9 & $-31.5 \pm 3.1$ & $-24.4 \pm 2.5$ & $-106.6 \pm 3.4$ & $-33.2 \pm 2.5$ & $-76.4 \pm 8.3$ \\
\hline 11 & $2 \mathrm{P} 95$ & -12.8 & $-28.5 \pm 2.5$ & $-25.6 \pm 2.3$ & $-101.8 \pm 3.3$ & $-32.8 \pm 2.7$ & $-70.3 \pm 7.9$ \\
\hline 12 & $1 \mathrm{MQ5}$ & -12.4 & $-33.8 \pm 3.5$ & $-22.9 \pm 2.2$ & $-117.4 \pm 6.4$ & $-36 \pm 2.8$ & $-86 \pm 11.6$ \\
\hline 13 & $2 \mathrm{~J} 4 \mathrm{I}$ & -12.4 & $-53.4 \pm 4.6$ & $-37.3 \pm 2.5$ & $-122.2 \pm 5.3$ & $-31 \pm 2.9$ & $-98.2 \pm 11.2$ \\
\hline 14 & $2 \mathrm{P} 93$ & -12.1 & $-26.8 \pm 2.7$ & $-23 \pm 2.2$ & $-97.8 \pm 3.9$ & $-29.6 \pm 2.9$ & $-69.9 \pm 8.9$ \\
\hline 15 & $2 \mathrm{BOH}$ & -11.7 & $-17.8 \pm 2.8$ & $-17.5 \pm 1.9$ & $-116.2 \pm 3.8$ & $-39.1 \pm 2.6$ & $-77.3 \pm 8.4$ \\
\hline 16 & $2 \mathrm{BQW}$ & -11.7 & $-28.5 \pm 2.4$ & $-23.4 \pm 2.3$ & $-116.9 \pm 4.5$ & $-31.2 \pm 2.9$ & $-87.9 \pm 9.5$ \\
\hline
\end{tabular}




\begin{tabular}{|c|c|c|c|c|c|c|c|}
\hline 17 & $2 \mathrm{~J} 95$ & -11.5 & $-39.6 \pm 3.8$ & $-26.8 \pm 2.4$ & $-114.6 \pm 3.2$ & $-29.4 \pm 3$ & $-90.7 \pm 8.8$ \\
\hline 18 & $2 \mathrm{~J} 34$ & -10.7 & $-32.4 \pm 3.1$ & $-28 \pm 2.5$ & $-106.8 \pm 3.9$ & $-31.7 \pm 2.9$ & $-76.9 \pm 9.2$ \\
\hline 19 & $1 \mathrm{FAX}$ & -10.1 & $-56.7 \pm 5.4$ & $-42.2 \pm 3.1$ & $-100.3 \pm 6.4$ & $-28.9 \pm 3.5$ & $-76.8 \pm 13$ \\
\hline 20 & $2 \mathrm{~J} 38$ & -10.0 & $-29.1 \pm 5.6$ & $-25.7 \pm 2.6$ & $-97.8 \pm 5.2$ & $-29.6 \pm 3.1$ & $-69.6 \pm 11.9$ \\
\hline 21 & $2 \mathrm{BQ} 7$ & -9.7 & $-19 \pm 3.1$ & $-18.8 \pm 2.1$ & $-105 \pm 3.4$ & $-30.8 \pm 2.5$ & $-74.3 \pm 8.1$ \\
\hline 22 & $2 \mathrm{UWP}$ & -9.4 & $-38.1 \pm 3.6$ & $-26.9 \pm 3$ & $-101.6 \pm 3.9$ & $-28.2 \pm 2.7$ & $-78.2 \pm 9.4$ \\
\hline 23 & 2J94 & -8.6 & $-42.8 \pm 3.3$ & $-37.9 \pm 2.8$ & $-105.2 \pm 4.3$ & $-31.1 \pm 3.3$ & $-76.2 \pm 10.2$ \\
\hline
\end{tabular}

Table S17 - Average polar and non-polar interaction energy in the bound and free state for 23 Thrombin complexes. Absolute binding free energy $\Delta G_{\mathrm{LIE}}$ was obtained for $\alpha=0.16$ (optimal value for a set of 23 compounds).

\begin{tabular}{|c|c|c|c|c|c|c|c|}
\hline No. & $\begin{array}{l}\text { Thrombin } \\
\text { PDB ID }\end{array}$ & $\Delta G_{\text {exp }}$ & $\left\langle V^{\text {elec }}\right\rangle_{\text {bound }}$ & $\left\langle V^{\text {elec }}\right\rangle_{\text {free }}$ & $\left\langle V^{\mathrm{vdw}}\right\rangle_{\text {bound }}$ & $\left\langle V^{\mathrm{vdw}}\right\rangle_{\text {free }}$ & $\begin{array}{c}\Delta G_{\mathrm{LIE}} \\
\alpha=0.16\end{array}$ \\
\hline 1 & 1SL3 & -16.3 & $-48.4 \pm 3.5$ & $-33.1 \pm 3.1$ & $-114.3 \pm 5.9$ & $-34.9 \pm 3.1$ & $-20.3 \pm 4.8$ \\
\hline 2 & $1 \mathrm{C} 4 \mathrm{~V}$ & -14.8 & $-47.5 \pm 3.8$ & $-38 \pm 2.9$ & $-114 \pm 6$ & $-35.5 \pm 3.7$ & $-16.7 \pm 4.4$ \\
\hline 3 & $1 \mathrm{C} 4 \mathrm{U}$ & -14.2 & $-52.7 \pm 4.7$ & $-35.1 \pm 3.1$ & $-62.4 \pm 4.5$ & $-17.3 \pm 3.2$ & $-14.8 \pm 4.6$ \\
\hline 4 & $2 \mathrm{ZO} 3$ & -13.7 & $-47.4 \pm 4.5$ & $-40.4 \pm 3.3$ & $-96.9 \pm 5.9$ & $-32.1 \pm 3.5$ & $-13.3 \pm 4.9$ \\
\hline 5 & 1 TA6 & -12.5 & $-31.4 \pm 3.7$ & $-30.3 \pm 2.7$ & $-110.1 \pm 5.5$ & $-34.6 \pm 2.9$ & $-12.6 \pm 4.1$ \\
\hline 6 & 1NT1 & -12.2 & $-39.4 \pm 3.9$ & $-30.9 \pm 2.6$ & $-110.6 \pm 5.5$ & $-33.8 \pm 3$ & $-15.9 \pm 4.1$ \\
\hline 7 & $1 \mathrm{BMN}$ & -11.6 & $-43.4 \pm 5.7$ & $-47.7 \pm 3.3$ & $-110.4 \pm 6.9$ & $-28.8 \pm 3.6$ & $-11.5 \pm 5$ \\
\hline 8 & 1ETS & -11.3 & $-50.1 \pm 6.1$ & $-41.2 \pm 3.8$ & $-103.5 \pm 7.8$ & $-31.9 \pm 3.5$ & $-15.3 \pm 6.1$ \\
\hline 9 & 2JH6 & -10.7 & $-31.2 \pm 4$ & $-29.3 \pm 3.6$ & $-101.9 \pm 4.7$ & $-29.4 \pm 3.3$ & $-12.4 \pm 4.5$ \\
\hline 10 & 3DHK & -10.0 & $-28.9 \pm 4.1$ & $-27.6 \pm 2.4$ & $-99.4 \pm 4.8$ & $-33.8 \pm 2.7$ & $-11 \pm 4$ \\
\hline 11 & $3 \mathrm{DUX}$ & -9.6 & $-18.5 \pm 3.3$ & $-21.1 \pm 2.4$ & $-98.5 \pm 5.4$ & $-30.1 \pm 2.6$ & $-9.8 \pm 3.7$ \\
\hline 12 & $2 \mathrm{ZC} 9$ & -9.2 & $-24.8 \pm 3.9$ & $-24.5 \pm 2.5$ & $-85.5 \pm 5.5$ & $-27.7 \pm 2.5$ & $-9.3 \pm 4.1$ \\
\hline 13 & $2 Z G X$ & -9.2 & $-30.2 \pm 3.9$ & $-35.8 \pm 3$ & $-85.2 \pm 5$ & $-22.1 \pm 2.8$ & $-7.7 \pm 4.2$ \\
\hline 14 & $2 \mathrm{JH} 5$ & -9.0 & $-31.9 \pm 3.3$ & $-27.9 \pm 2.9$ & $-101.7 \pm 5$ & $-30.2 \pm 2.9$ & $-13.2 \pm 3.9$ \\
\hline 15 & $1 \mathrm{AWH}$ & -8.3 & $-42.4 \pm 4.1$ & $-36.4 \pm 2.9$ & $-98.7 \pm 6.3$ & $-31.2 \pm 3.6$ & $-12.8 \pm 3.9$ \\
\hline 16 & $1 \mathrm{D} 6 \mathrm{~W}$ & -8.2 & $-53.2 \pm 5.4$ & $-46.9 \pm 3.2$ & $-104 \pm 7.1$ & $-30.7 \pm 3.4$ & $-14.4 \pm 5.4$ \\
\hline 17 & $3 \mathrm{SHC}$ & -7.8 & $-25.8 \pm 3.3$ & $-30 \pm 2.8$ & $-87.7 \pm 4.6$ & $-27 \pm 3$ & $-7.9 \pm 3.8$ \\
\hline 18 & $3 \mathrm{SHA}$ & -7.7 & $-26.7 \pm 3.3$ & $-28.2 \pm 2.9$ & $-87.8 \pm 5.3$ & $-27 \pm 2.8$ & $-9.1 \pm 4$ \\
\hline 19 & $2 \mathrm{ZFP}$ & -7.1 & $-24.8 \pm 3.8$ & $-21.8 \pm 2.3$ & $-74.8 \pm 5.9$ & $-22.8 \pm 2.6$ & $-9.6 \pm 4$ \\
\hline 20 & $3 \mathrm{~F} 68$ & -6.9 & $-21 \pm 4.4$ & $-19 \pm 2.2$ & $-100 \pm 5.5$ & $-32.4 \pm 2.3$ & $-11.7 \pm 4.1$ \\
\hline 21 & 3EGK & -6.4 & $-21.4 \pm 2.4$ & $-20.8 \pm 2.4$ & $-90.5 \pm 5.4$ & $-32.4 \pm 2.7$ & $-9.5 \pm 3.4$ \\
\hline 22 & $3 \mathrm{P} 17$ & -6.1 & $-24.3 \pm 4$ & $-31.9 \pm 2.6$ & $-79.7 \pm 5$ & $-25.5 \pm 3.1$ & $-5.4 \pm 4.2$ \\
\hline 23 & $3 \mathrm{SV} 2$ & -5.8 & $-27.1 \pm 4.3$ & $-30.7 \pm 2.6$ & $-78.2 \pm 5.1$ & $-25.5 \pm 3.2$ & $-6.9 \pm 4.3$ \\
\hline
\end{tabular}

Table S18 - Average polar and non-polar interaction energy in the bound and free state for $23 \mathrm{HIV}-1$ complexes. Absolute binding free energy $\Delta G_{\mathrm{LIE}}$ Was obtained for $\alpha=0.16$ (optimal value for a set of 23 compounds). 


\begin{tabular}{|c|c|c|c|c|c|c|c|}
\hline No. & $\begin{array}{c}\text { HIV-1 } \\
\text { PDB ID }\end{array}$ & $\Delta G_{\text {exp }}$ & $\left\langle V^{\text {elec }}\right\rangle_{\text {bound }}$ & $\left\langle V^{\text {elec }}\right\rangle_{\text {free }}$ & $\left\langle V^{\text {vdw }}\right\rangle_{\text {bound }}$ & $\left\langle V^{\text {vdw }}\right\rangle_{\text {free }}$ & $\Delta=0.43$ \\
\hline 1 & $1 \mathrm{HVK}$ & -15.04 & $-72.8 \pm 6.4$ & $-85.8 \pm 4.0$ & $-103.5 \pm 10.9$ & $-48.6 \pm 4.7$ & $-19.3 \pm 10.2$ \\
\hline 2 & $1 \mathrm{HVL}$ & -13.66 & $-73.8 \pm 6.2$ & $-82.5 \pm 4.4$ & $-101.8 \pm 10.8$ & $-49.3 \pm 4.7$ & $-19.7 \pm 10.1$ \\
\hline 3 & $1 \mathrm{EBY}$ & -13.31 & $-63.7 \pm 6.4$ & $-81.3 \pm 2.5$ & $-88.4 \pm 11.8$ & $-39.9 \pm 3.4$ & $-15.0 \pm 9.5$ \\
\hline 4 & $1 \mathrm{C} 6 \mathrm{Y}$ & -13.05 & $-55.4 \pm 5.2$ & $-73.8 \pm 4.3$ & $-84.1 \pm 8.5$ & $-40.9 \pm 5.1$ & $-12.5 \pm 9.0$ \\
\hline 5 & $2 \mathrm{BPX}$ & -12.9 & $-70.2 \pm 6.9$ & $-68.3 \pm 3.9$ & $-78.3 \pm 7.7$ & $-41.3 \pm 4.0$ & $-16.5 \pm 8.6$ \\
\hline 6 & $1 \mathrm{EC} 3$ & -12.4 & $-87.9 \pm 8.0$ & $-83.4 \pm 4.4$ & $-110.5 \pm 11.6$ & $-50.3 \pm 3.9$ & $-27.4 \pm 10.7$ \\
\hline 7 & $1 \mathrm{EC} 1$ & -12.24 & $-65.8 \pm 6.1$ & $-86.4 \pm 3.6$ & $-94.1 \pm 9.6$ & $-49.4 \pm 4.4$ & $-12.4 \pm 9.2$ \\
\hline 8 & $2 \mathrm{CEJ}$ & -11.83 & $-57.5 \pm 8.3$ & $-71.8 \pm 3.6$ & $-71.7 \pm 8.1$ & $-40.1 \pm 3.1$ & $-8.9 \pm 8.7$ \\
\hline 9 & $2 \mathrm{UXZ}$ & -11.64 & $-57.6 \pm 4.3$ & $-77.7 \pm 3.2$ & $-81.6 \pm 7.7$ & $-40.7 \pm 5.1$ & $-11.0 \pm 8.0$ \\
\hline 10 & $1 \mathrm{D} 4 \mathrm{~J}$ & -11.47 & $-65.4 \pm 5.0$ & $-76.3 \pm 3.5$ & $-76.9 \pm 9.1$ & $-42.3 \pm 3.7$ & $-11.3 \pm 8.3$ \\
\hline 11 & $2 \mathrm{CEN}$ & -11.39 & $-57.3 \pm 6.7$ & $-77.5 \pm 3.4$ & $-89.0 \pm 9.2$ & $-41.7 \pm 4.0$ & $-13.7 \pm 9.0$ \\
\hline 12 & $1 \mathrm{G} 35$ & -11.17 & $-68.7 \pm 5.6$ & $-71.4 \pm 3.4$ & $-73.1 \pm 7.6$ & $-40.8 \pm 3.7$ & $-13.0 \pm 7.8$ \\
\hline 13 & $2 \mathrm{BQV}$ & -11.04 & $-55.8 \pm 5.8$ & $-69.5 \pm 3.2$ & $-75.6 \pm 7.3$ & $-36.7 \pm 3.1$ & $-12.2 \pm 7.5$ \\
\hline 14 & $1 \mathrm{SBG}$ & -10.63 & $-42.9 \pm 6.6$ & $-64.3 \pm 3.2$ & $-73.9 \pm 8.6$ & $-32.7 \pm 3.2$ & $-9.8 \pm 8.7$ \\
\hline 15 & $2 \mathrm{BPV}$ & -10.53 & $-58.7 \pm 4.9$ & $-74.7 \pm 3.2$ & $-83.1 \pm 9.7$ & $-42.9 \pm 4.0$ & $-12.0 \pm 8.6$ \\
\hline 16 & $2 \mathrm{BPY}$ & -10.16 & $-71.8 \pm 5.4$ & $-82.9 \pm 3.8$ & $-89.2 \pm 9.6$ & $-48.7 \pm 4.2$ & $-13.7 \pm 9.0$ \\
\hline 17 & $2 \mathrm{UPJ}$ & -10.14 & $-60.7 \pm 7.0$ & $-61.9 \pm 3.8$ & $-64.0 \pm 6.1$ & $-41.5 \pm 3.5$ & $-9.2 \pm 8.1$ \\
\hline 18 & $2 \mathrm{UY} 0$ & -9.5 & $-45.7 \pm 7.0$ & $-80.8 \pm 3.5$ & $-82.4 \pm 7.9$ & $-41.5 \pm 3.9$ & $-6.0 \pm 8.5$ \\
\hline 19 & $2 \mathrm{PWR}$ & -9.04 & $-61.9 \pm 6.4$ & $-66.5 \pm 3.8$ & $-89.9 \pm 8.2$ & $-35.9 \pm 3.8$ & $-21.2 \pm 9.6$ \\
\hline 20 & $2 \mathrm{PWC}$ & -9.02 & $-53.0 \pm 5.9$ & $-61.1 \pm 2.9$ & $-79.2 \pm 8.4$ & $-29.4 \pm 3.8$ & $-17.9 \pm 9.0$ \\
\hline 21 & $1 \mathrm{HBV}$ & -8.74 & $-70.6 \pm 8.4$ & $-64.0 \pm 4.5$ & $-74.6 \pm 8.6$ & $-38.8 \pm 4.0$ & $-18.2 \pm 11.0$ \\
\hline 22 & $3 \mathrm{BGB}$ & -8.3 & $-48.7 \pm 5.1$ & $-63.1 \pm 2.9$ & $-76.7 \pm 6.5$ & $-34.2 \pm 4.0$ & $-12.1 \pm 8.0$ \\
\hline 23 & $3 \mathrm{BGC}$ & -6.89 & $-47.4 \pm 7.4$ & $-69.0 \pm 3.4$ & $-86.2 \pm 8.3$ & $-35.0 \pm 5.2$ & $-12.7 \pm 10.4$ \\
\hline
\end{tabular}

Table S19 - Average polar and non-polar interaction energy in the bound and free state for 23 MCL-1 complexes. Absolute binding free energy $\Delta G_{\text {LIE }}$ was obtained for $\alpha=0.16$ (optimal value for a set of 23 compounds) and $\alpha=0.11$ (optimal value for a combined set of 69 compounds).

\begin{tabular}{|c|c|c|c|c|c|c|c|}
\hline No. & $\begin{array}{c}\text { MCL-1 } \\
\text { PDB ID }\end{array}$ & $\Delta G_{\text {exp }}$ & $\left\langle V^{\text {elec }}\right\rangle_{\text {bound }}$ & $\left\langle V^{\text {elec }}\right\rangle_{\text {free }}$ & $\left\langle V^{\mathrm{vdw}}\right\rangle_{\text {bound }}$ & $\left\langle V^{\mathrm{vdw}}\right\rangle_{\text {free }}$ & $\begin{array}{c}\Delta G_{\text {LIE }} \\
\alpha=1\end{array}$ \\
\hline 1 & $6 \mathrm{NE} 5$ & -14.44 & $-130.1 \pm 3.0$ & $-140.8 \pm 7.6$ & $-60.5 \pm 2.0$ & $-35.2 \pm 4.0$ & $-21.3 \pm 10.0$ \\
\hline 2 & $6 \mathrm{OQC}$ & -14.27 & $-45.4 \pm 2.6$ & $-56.5 \pm 5.8$ & $-69.4 \pm 1.4$ & $-39.2 \pm 3.5$ & $-26.1 \pm 8.0$ \\
\hline 3 & $6 \mathrm{OQB}$ & -14.14 & $-45.9 \pm 2.8$ & $-57.0 \pm 5.8$ & $-69.4 \pm 1.6$ & $-40.6 \pm 3.3$ & $-24.7 \pm 8.0$ \\
\hline 4 & $6 \mathrm{UDV}$ & -14.13 & $-53.9 \pm 3.2$ & $-61.8 \pm 6.2$ & $-69.4 \pm 2.0$ & $-40.8 \pm 3.8$ & $-25.7 \pm 9.2$ \\
\hline 5 & $6 \mathrm{UD} 2$ & -14.01 & $-57.2 \pm 7.7$ & $-71.8 \pm 9.8$ & $-89.5 \pm 1.6$ & $-57.1 \pm 3.9$ & $-27.1 \pm 12.0$ \\
\hline 6 & $6 \mathrm{UDI}$ & -13.73 & $-49.4 \pm 4.7$ & $-62.4 \pm 7.8$ & $-75.3 \pm 1.8$ & $-47.5 \pm 3.7$ & $-22.9 \pm 10.1$ \\
\hline 7 & $6 \mathrm{UDT}$ & -13.73 & $-46.3 \pm 5.8$ & $-51.3 \pm 7.4$ & $-64.6 \pm 2.7$ & $-39.8 \pm 3.6$ & $-23.1 \pm 10.6$ \\
\hline 8 & $6 \mathrm{OQD}$ & -13.53 & $-45.4 \pm 2.6$ & $-57.8 \pm 5.9$ & $-67.2 \pm 1.5$ & $-39.9 \pm 3.5$ & $-22.7 \pm 8.1$ \\
\hline
\end{tabular}




\begin{tabular}{|c|c|c|c|c|c|c|c|}
\hline 9 & $5 F D R$ & -12.9 & $-86.0 \pm 2.6$ & $-96.4 \pm 7.4$ & $-54.4 \pm 1.9$ & $-35.6 \pm 4.5$ & $-15.0 \pm 10.0$ \\
\hline 10 & 6 UDU & -12.77 & $-48.0 \pm 10.0$ & $-49.5 \pm 5.8$ & $-62.1 \pm 1.9$ & $-35.8 \pm 3.4$ & $-25.8 \pm 10.5$ \\
\hline 11 & $6 \mathrm{BW} 8$ & -12.61 & $-53.6 \pm 1.9$ & $-62.4 \pm 6.1$ & $-68.9 \pm 1.6$ & $-42.7 \pm 3.8$ & $-22.9 \pm 8.3$ \\
\hline 12 & $6 \mathrm{~B} 4 \mathrm{U}$ & -11.04 & $-32.4 \pm 6.4$ & $-51.2 \pm 5.6$ & $-59.3 \pm 2.7$ & $-36.8 \pm 3.6$ & $-15.5 \pm 10.7$ \\
\hline 13 & $6 \mathrm{BW} 2$ & -10.54 & $-49.6 \pm 3.9$ & $-65.9 \pm 6.0$ & $-63.7 \pm 3.3$ & $-40.2 \pm 3.4$ & $-17.4 \pm 10.4$ \\
\hline 14 & $5 \mathrm{IEZ}$ & -10.48 & $-73.3 \pm 2.7$ & $-75.7 \pm 8.0$ & $-53.6 \pm 1.6$ & $-34.8 \pm 4.1$ & $-17.9 \pm 9.7$ \\
\hline 15 & $6 \mathrm{QYP}$ & -10.07 & $-52.1 \pm 3.6$ & $-52.6 \pm 5.3$ & $-50.8 \pm 2.0$ & $-36.3 \pm 3.3$ & $-14.3 \pm 8.2$ \\
\hline 16 & $6 \mathrm{QZ8}$ & -10.01 & $-48.3 \pm 3.6$ & $-54.0 \pm 5.8$ & $-44.8 \pm 1.4$ & $-26.4 \pm 3.2$ & $-16.5 \pm 7.8$ \\
\hline 17 & $4 \mathrm{HW} 2$ & -9.96 & $-41.2 \pm 1.7$ & $-45.0 \pm 4.7$ & $-42.6 \pm 1.7$ & $-24.4 \pm 2.8$ & $-16.8 \pm 6.9$ \\
\hline 18 & $5 \mathrm{FC} 4$ & -9.11 & $-53.0 \pm 2.5$ & $-58.6 \pm 5.5$ & $-43.7 \pm 2.2$ & $-29.9 \pm 3.0$ & $-11.4 \pm 8.7$ \\
\hline 19 & $4 \mathrm{ZBI}$ & -8.93 & $-30.2 \pm 2.2$ & $-37.2 \pm 4.9$ & $-46.8 \pm 1.2$ & $-27.0 \pm 2.7$ & $-17.2 \pm 6.6$ \\
\hline 20 & $4 \mathrm{HW} 3$ & -8.91 & $-24.1 \pm 2.7$ & $-31.6 \pm 4.7$ & $-45.9 \pm 2.0$ & $-26.1 \pm 2.8$ & $-17.0 \pm 7.6$ \\
\hline 21 & $6 \mathrm{~B} 4 \mathrm{~L}$ & -8.88 & $-34.0 \pm 3.0$ & $-48.9 \pm 5.7$ & $-42.0 \pm 1.4$ & $-23.0 \pm 2.9$ & $-13.4 \pm 7.6$ \\
\hline 22 & 5FDO & -8.84 & $-50.7 \pm 2.6$ & $-58.4 \pm 5.1$ & $-53.5 \pm 1.2$ & $-34.0 \pm 3.4$ & $-16.2 \pm 7.9$ \\
\hline 23 & $6 \mathrm{QZB}$ & -7.71 & $-31.2 \pm 4.2$ & $-35.8 \pm 7.8$ & $-44.0 \pm 1.3$ & $-28.5 \pm 3.1$ & $-13.8 \pm 8.8$ \\
\hline
\end{tabular}

Table S20 - Average polar and non-polar interaction energy in the bound and free state for 23 CDK- 2 complexes. Absolute binding free energy $\Delta G_{\text {LIE }}$ was obtained for $\alpha=0.16$ (optimal value for a set of 23 compounds) and $\alpha=0.11$ (optimal value for a combined set of 69 compounds).

\begin{tabular}{|c|c|c|c|c|c|c|c|}
\hline No. & $\begin{array}{c}\text { CDK-2 } \\
\text { PDB ID }\end{array}$ & $\Delta G_{\text {exp }}$ & $\left\langle V^{\text {elec }}\right\rangle_{\text {bound }}$ & $\left\langle V^{\text {elec }}\right\rangle_{\text {free }}$ & $\left\langle V^{\mathrm{vdw}}\right\rangle_{\text {bound }}$ & $\left\langle V^{\mathrm{vdw}}\right\rangle_{\text {free }}$ & $\begin{array}{c}\Delta G_{\text {LIE }} \\
\alpha=1\end{array}$ \\
\hline 1 & $2 \mathrm{FVD}$ & -11.7 & $-39.3 \pm 5.4$ & $-61.1 \pm 7.6$ & $-55.3 \pm 3.2$ & $-29.7 \pm 4.4$ & $-16.1 \pm 13.2$ \\
\hline 2 & $4 \mathrm{BCK}$ & -11.53 & $-56.7 \pm 6.8$ & $-63.2 \pm 8.8$ & $-43.2 \pm 4.1$ & $-27.2 \pm 4.5$ & $-13.3 \pm 15.3$ \\
\hline 3 & $4 \mathrm{BCN}$ & -10.87 & $-40.2 \pm 5.1$ & $-43.9 \pm 6.2$ & $-41.7 \pm 3.5$ & $-37.0 \pm 3.8$ & $-3.3 \pm 11.6$ \\
\hline 4 & $1 \mathrm{PF} 8$ & -10.31 & $-20.9 \pm 3.4$ & $-37.3 \pm 6.3$ & $-35.0 \pm 2.4$ & $-19.7 \pm 3.6$ & $-8.2 \pm 10.2$ \\
\hline 5 & $5 \mathrm{D} 1 \mathrm{~J}$ & -10.18 & $-36.3 \pm 4.8$ & $-41.5 \pm 6.4$ & $-48.0 \pm 3.5$ & $-33.1 \pm 3.9$ & $-12.7 \pm 12.2$ \\
\hline 6 & $1 \mathrm{PXM}$ & -9.91 & $-32.6 \pm 5.3$ & $-36.3 \pm 5.7$ & $-37.6 \pm 3.6$ & $-23.3 \pm 3.5$ & $-12.9 \pm 11.2$ \\
\hline 7 & $1 \mathrm{PXN}$ & -9.82 & $-37.4 \pm 5.1$ & $-41.3 \pm 6.6$ & $-39.9 \pm 3.5$ & $-23.9 \pm 3.5$ & $-14.6 \pm 11.4$ \\
\hline 8 & $4 \mathrm{BCM}$ & -9.48 & $-32.3 \pm 6.0$ & $-54.4 \pm 7.0$ & $-58.0 \pm 3.7$ & $-37.9 \pm 4.0$ & $-10.5 \pm 13.3$ \\
\hline 9 & $4 \mathrm{BCO}$ & -9.45 & $-40.6 \pm 5.9$ & $-57.4 \pm 7.0$ & $-61.0 \pm 3.8$ & $-36.7 \pm 4.0$ & $-17.1 \pm 13.4$ \\
\hline 10 & $4 \mathrm{BCQ}$ & -9.38 & $-47.2 \pm 6.7$ & $-53.5 \pm 7.0$ & $-45.0 \pm 3.8$ & $-32.4 \pm 4.1$ & $-9.9 \pm 13.8$ \\
\hline 11 & $4 \mathrm{ACM}$ & -9.17 & $-61.4 \pm 8.9$ & $-72.5 \pm 8.4$ & $-54.2 \pm 4.5$ & $-33.5 \pm 4.6$ & $-16.0 \pm 16.6$ \\
\hline 12 & $1 \mathrm{PXP}$ & -9.14 & $-19.7 \pm 4.4$ & $-34.6 \pm 5.5$ & $-47.4 \pm 3.3$ & $-27.6 \pm 3.1$ & $-13.4 \pm 10.6$ \\
\hline 13 & $1 \mathrm{PXL}$ & -8.97 & $-25.4 \pm 3.8$ & $-24.3 \pm 4.6$ & $-43.8 \pm 3.1$ & $-33.0 \pm 3.0$ & $-11.2 \pm 9.8$ \\
\hline 14 & $1 \mathrm{PYE}$ & -8.8 & $-31.9 \pm 5.3$ & $-44.5 \pm 6.5$ & $-39.2 \pm 3.3$ & $-26.4 \pm 4.1$ & $-7.3 \pm 12.5$ \\
\hline 15 & $4 \mathrm{EOR}$ & -8.65 & $-53.6 \pm 8.3$ & $-57.5 \pm 8.0$ & $-48.8 \pm 4.1$ & $-28.5 \pm 4.1$ & $-18.6 \pm 15.2$ \\
\hline 16 & $4 \mathrm{BCP}$ & -8.57 & $-66.6 \pm 7.3$ & $-67.5 \pm 7.7$ & $-48.4 \pm 4.1$ & $-32.3 \pm 5.2$ & $-15.7 \pm 15.8$ \\
\hline 17 & $4 \mathrm{EOS}$ & -8.3 & $-24.9 \pm 5.2$ & $-30.8 \pm 5.8$ & $-43.5 \pm 3.7$ & $-30.2 \pm 3.5$ & $-10.8 \pm 12.0$ \\
\hline 18 & $1 \mathrm{E} 1 \mathrm{X}$ & -8.08 & $-24.8 \pm 5.6$ & $-32.8 \pm 7.4$ & $-34.6 \pm 3.0$ & $-23.8 \pm 3.7$ & $-7.4 \pm 12.3$ \\
\hline 19 & $1 \mathrm{JSV}$ & -7.82 & $-57.5 \pm 7.4$ & $-55.1 \pm 9.2$ & $-30.0 \pm 4.0$ & $-16.6 \pm 4.4$ & $-14.5 \pm 15.6$ \\
\hline & & & & & & & \\
\hline
\end{tabular}




\begin{tabular}{|c|c|c|c|c|c|c|c|}
\hline 20 & 1 OGU & -7.71 & $-35.2 \pm 5.7$ & $-42.3 \pm 6.9$ & $-49.2 \pm 3.6$ & $-29.3 \pm 3.6$ & $-16.8 \pm 12.6$ \\
\hline 21 & 1 PXJ & -7.12 & $-24.5 \pm 3.6$ & $-25.6 \pm 5.2$ & $-30.6 \pm 2.4$ & $-16.5 \pm 2.8$ & $-13.6 \pm 9.0$ \\
\hline 22 & $1 \mathrm{E} 1 \mathrm{~V}$ & -6.75 & $-27.0 \pm 3.7$ & $-39.6 \pm 6.1$ & $-33.0 \pm 3.2$ & $-23.1 \pm 3.3$ & $-4.5 \pm 10.7$ \\
\hline 23 & $1 \mathrm{~V} 1 \mathrm{~K}$ & -6.12 & $-44.0 \pm 6.0$ & $-46.4 \pm 6.7$ & $-50.6 \pm 3.9$ & $-35.6 \pm 4.4$ & $-14.1 \pm 12.9$ \\
\hline
\end{tabular}

Table S21 - Minimum value of RMSED and amin for all studied data sets. Correlation coefficient $R$ at $\alpha \min$ is given in the last column.

\begin{tabular}{|l|l|l|l|}
\hline Dataset & $\begin{array}{l}\text { min RMSED } \\
(\mathrm{kcal} / \mathrm{mol})\end{array}$ & $\alpha_{\min }$ & $R$ at $\alpha_{\min }$ \\
\hline Beta-lactamase & 2.9 & 0.16 & 0.53 \\
\hline Factor Xa & 3.7 & 0.12 & 0.17 \\
\hline Thrombin & 3.1 & 0.13 & 0.80 \\
\hline HIV-1 & 4.2 & 0.35 & 0.23 \\
\hline MCL-1 & 1.4 & 0.61 & 0.78 \\
\hline CDK-2 & 46.4 & 0 & 0 \\
\hline All six sets & 7.5 & 0.19 & 0.28 \\
\hline $\begin{array}{l}\text { Combined set of thrombin, factor } \\
\text { Xa, beta-lactamase, and MCL-1 }\end{array}$ & 6.3 & 0.15 & 0.35 \\
\hline Combined set of HIV-1 and CDK-2 & 5.9 & 0.40 & 0.47 \\
\hline $\begin{array}{l}\text { Combined set of thrombin, factor } \\
\text { Xa, beta-lactamase }\end{array}$ & 4.4 & 0.13 & 0.85 \\
\hline
\end{tabular}



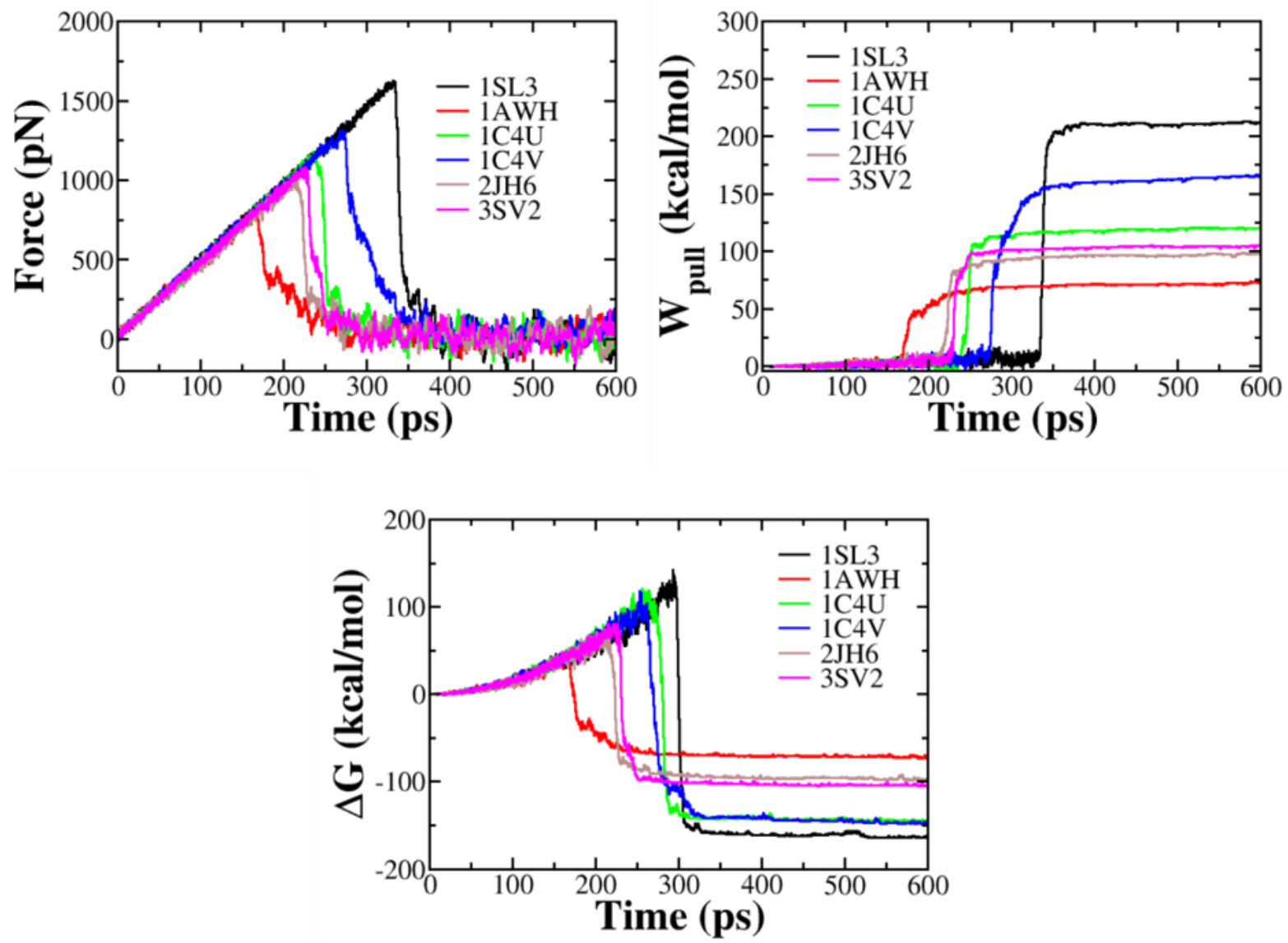

Figure S1 - Time dependence of the force, pulling work, and Jarzynski's binding free energy for six reference ligands of thrombin. Results were obtained in one SMD run. 

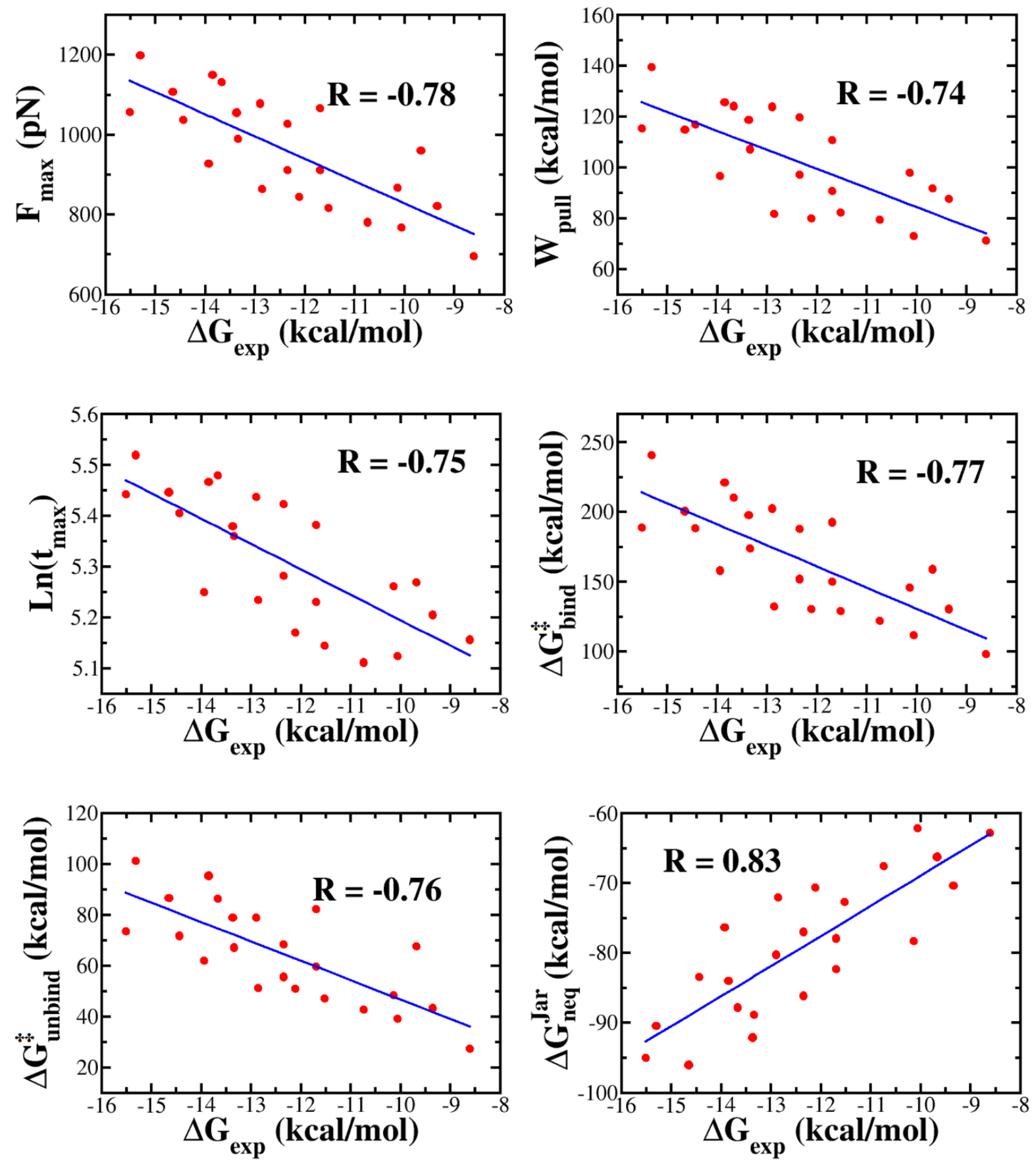

Figure S2 - Correlation between the experimental binding free energy $\Delta G_{\text {exp }}$ and the rupture force $\mathrm{F}_{\max }$, pulling work $\mathrm{W}_{\text {pull, }}$, rupture time $\mathrm{t}_{\max }$, binding $\Delta G_{\text {bind }}^{\ddagger}$ and unbinding $\Delta G_{\text {unbind }}^{\ddagger}$ barriers and Jarzynski's binding free energy $\Delta G_{\text {neq }}^{\text {Jar }}$, obtained in the SMD simulation for the factor Xa complexes. Correlation coefficient $\mathrm{R}$ is also shown. 

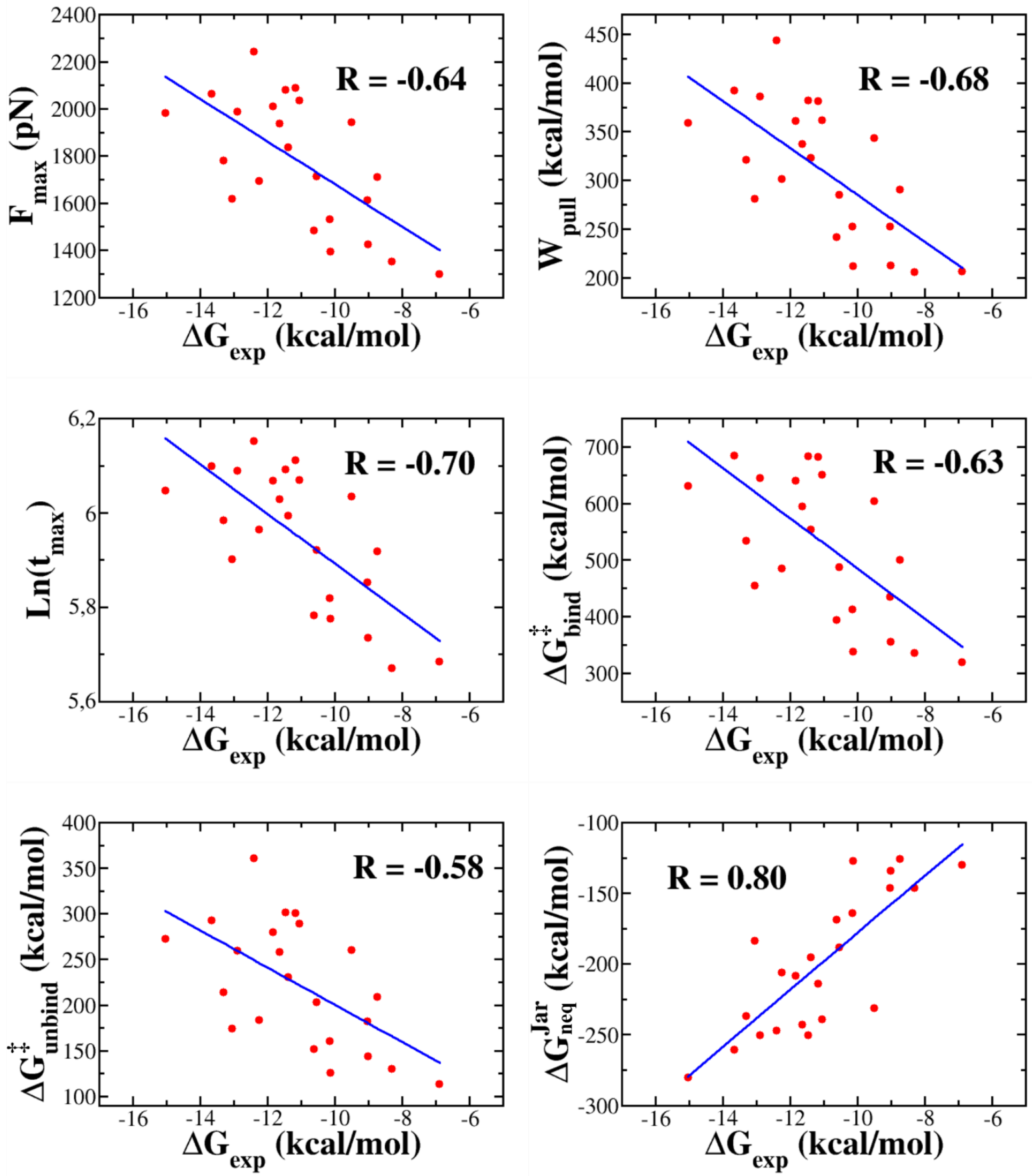

Figure S3 - Correlation between the experimental binding free energy $\Delta G_{\text {exp }}$ and the rupture force $\mathrm{F}_{\max }$, pulling work $\mathrm{W}_{\text {pull, }}$, rupture time $\mathrm{t}_{\max }$, binding $\Delta G_{\text {bind }}^{\ddagger}$ and unbinding $\Delta G_{\text {unbind }}^{\ddagger}$ barriers and Jarzynski's binding free energy $\Delta G_{n e q}^{J a r}$, obtained in the SMD simulation for the HIV-1 complexes. Correlation coefficient $\mathrm{R}$ is also shown. 

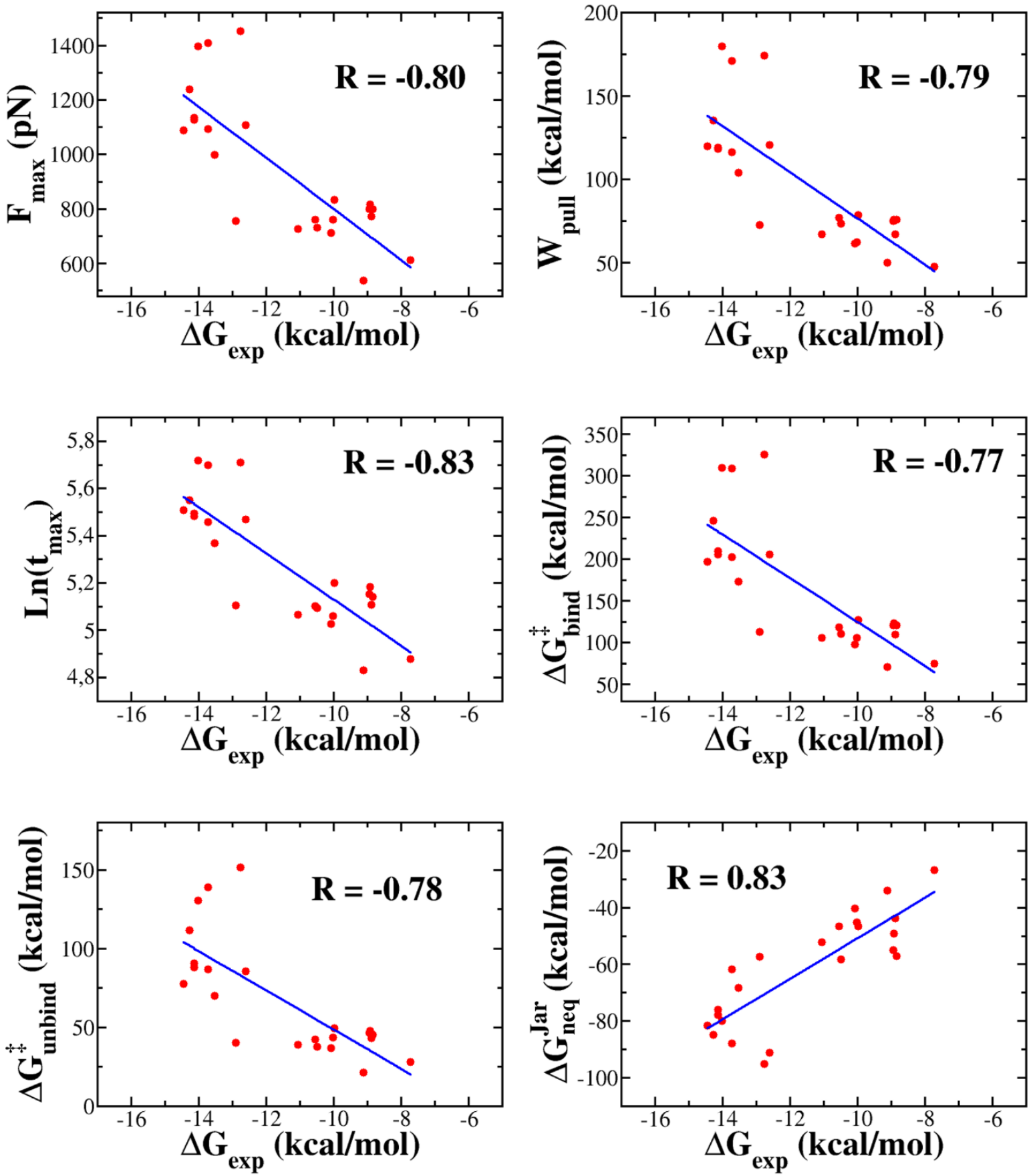

Figure S4 - Correlation between the experimental binding free energy $\Delta G_{\exp }$ and the rupture force $\mathrm{F}_{\max }$, pulling work $\mathrm{W}_{\text {pull, }}$, rupture time $\mathrm{t}_{\max }$, binding $\Delta G_{\text {bind }}^{\ddagger}$ and unbinding $\Delta G_{\text {unbind }}^{\ddagger}$ barriers and Jarzynski's binding free energy $\Delta G_{n e q}^{J a r}$, obtained in the SMD simulation for the MCL-1 complexes. Correlation coefficient $\mathrm{R}$ is also shown. 

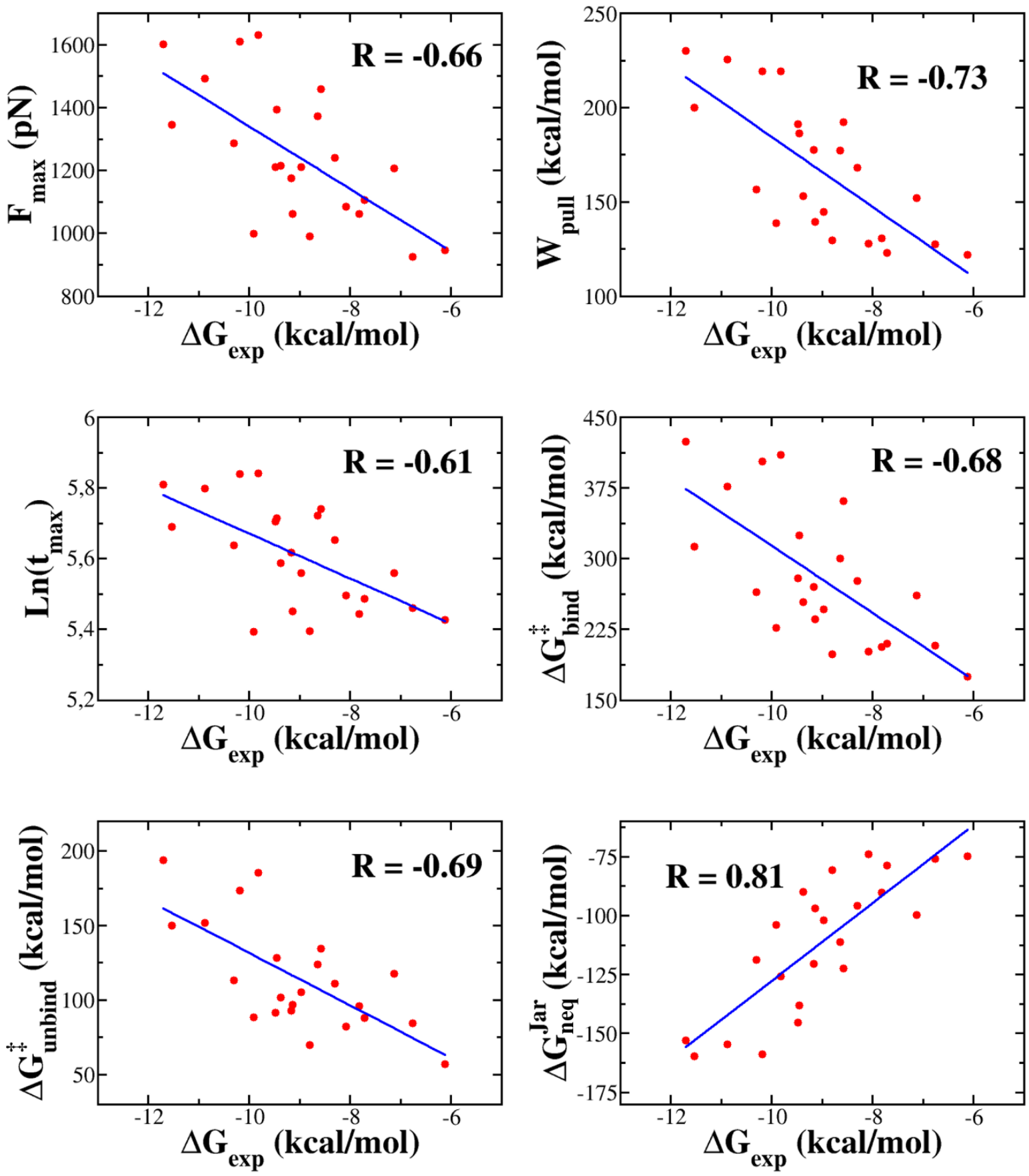

Figure S5 - Correlation between the experimental binding free energy $\Delta G_{\text {exp }}$ and the rupture force $\mathrm{F}_{\max }$, pulling work $\mathrm{W}_{\text {pull, }}$, rupture time $\mathrm{t}_{\max }$, binding $\Delta G_{\text {bind }}^{\ddagger}$ and unbinding $\Delta G_{\text {unbind }}^{\ddagger}$ barriers and Jarzynski's binding free energy $\Delta G_{n e q}^{J a r}$, obtained in the SMD simulation for the CDK-2 complexes. Correlation coefficient $\mathrm{R}$ is also shown. 


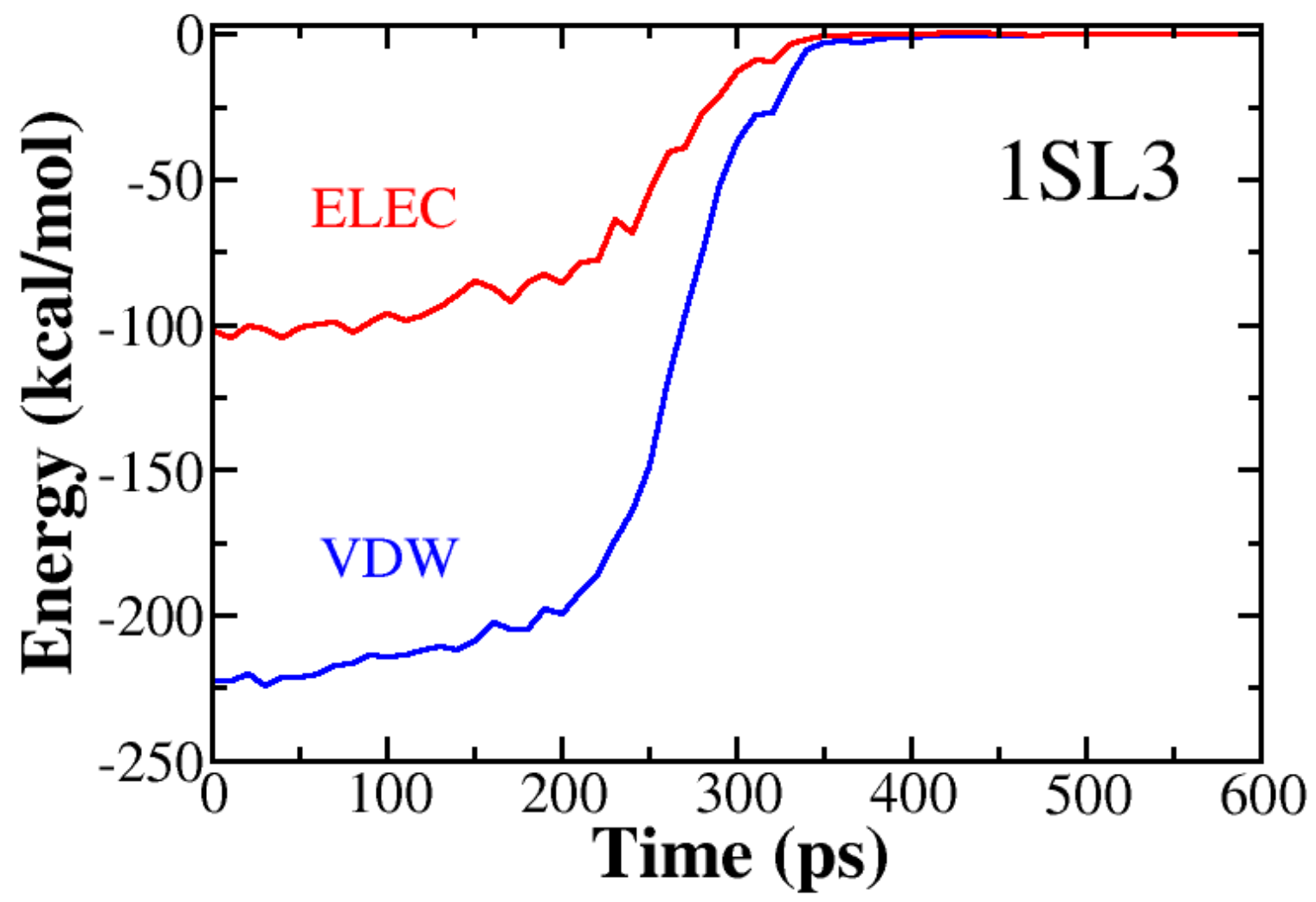

Figure S6 - The time dependence of polar and non-polar interaction energy between the ligand and protein in the absence of water for the 1SL3 complex from the thrombin set. The data were obtained by averaging over 20 trajectories. 


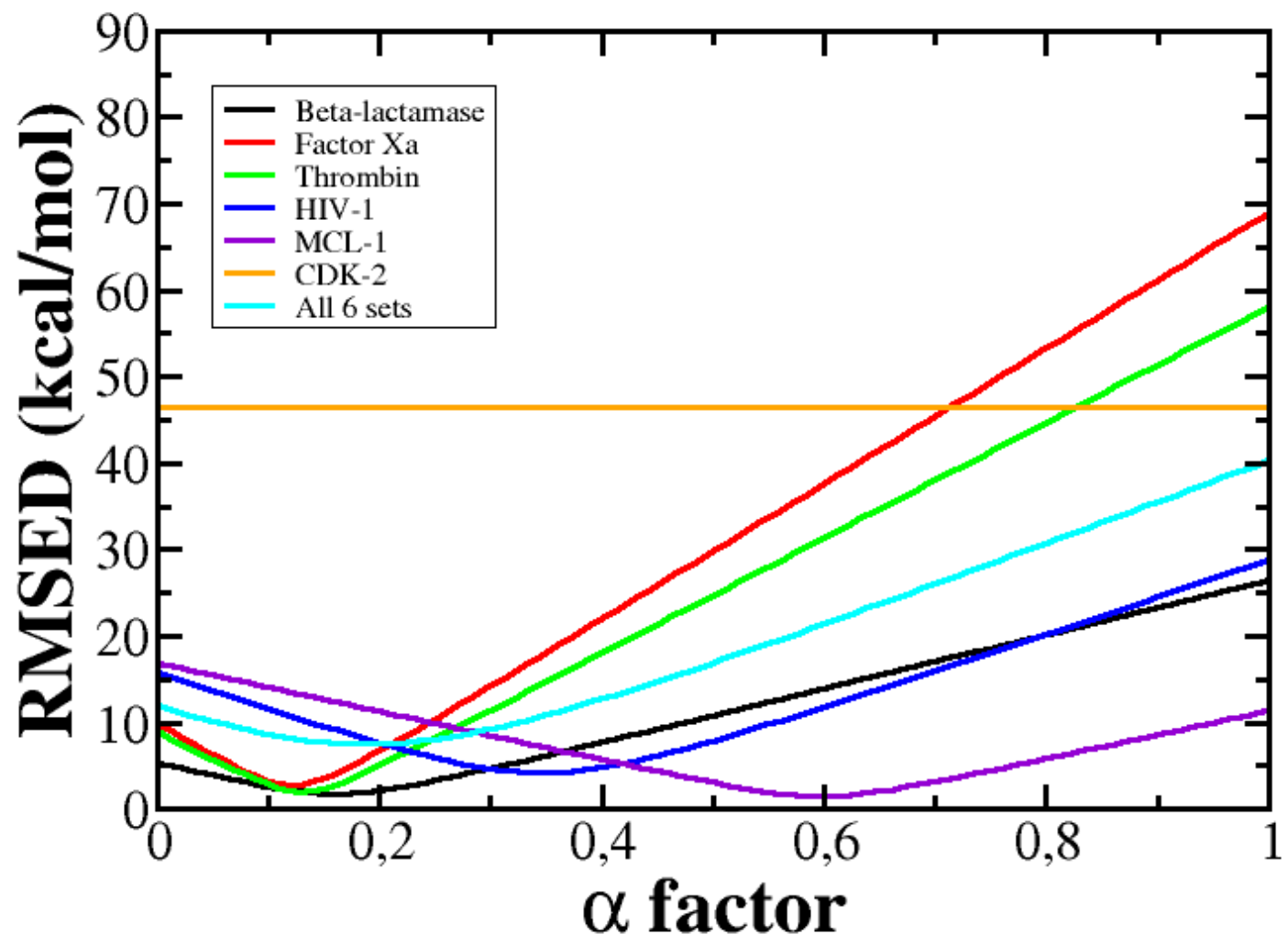

Figure S7 - Dependence of RMSED on $\alpha$ for six data sets and the combination of all 6 sets. 

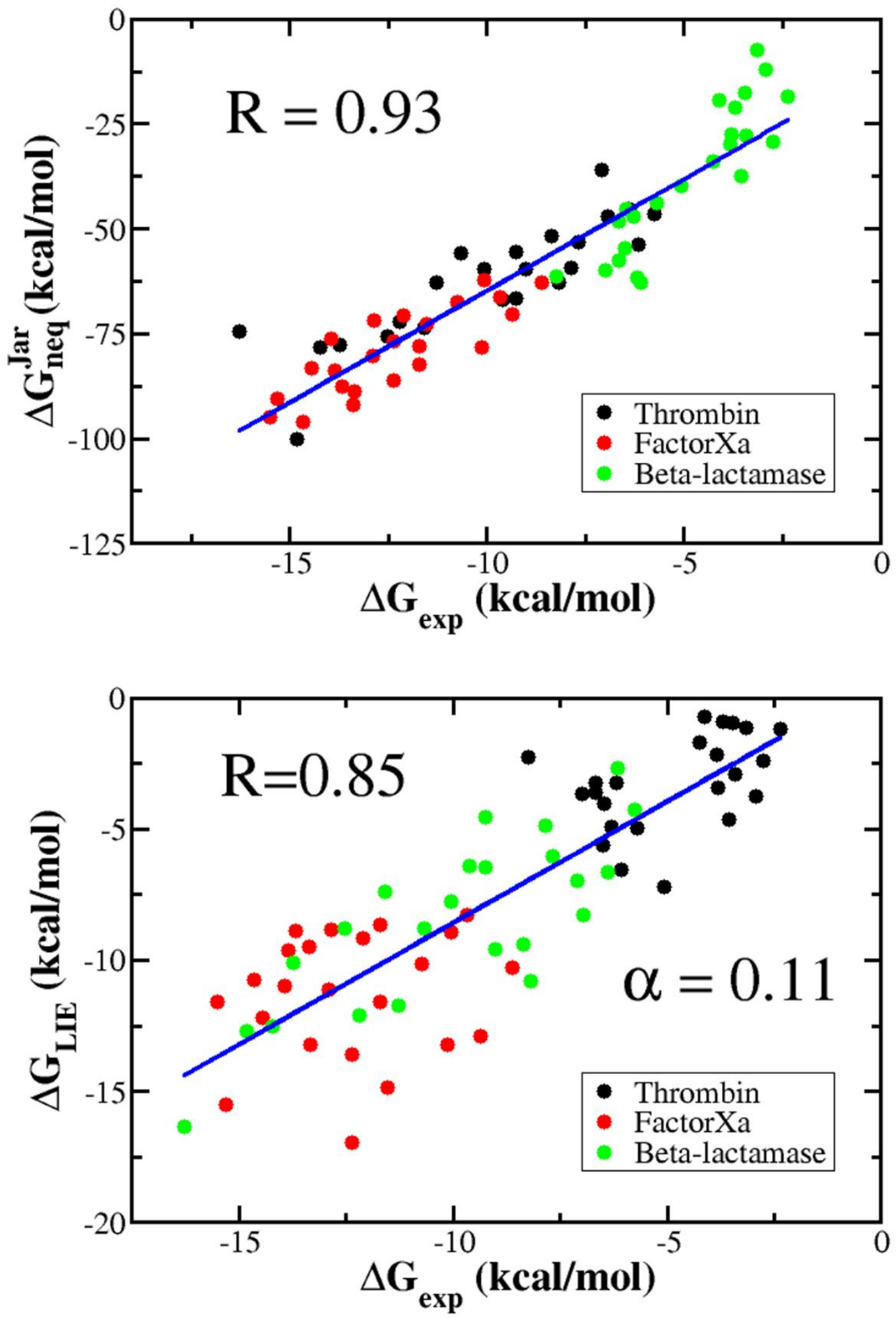

Figure S8. Correlation between the binding free energies of Jarzynski (upper panel) and LIE (lower panel) with the experimental binding free energy for 3 combined data sets including beta-lactamase, thrombin, and factor Xa. $\Delta G_{\mathrm{LIE}}$ was obtained using $\alpha=0.11$. 


\section{Reference}

1. Young, M.B., et al., Discovery and evaluation of potent P1 aryl heterocycle-based thrombin inhibitors. J Med Chem, 2004. 47(12): p. 2995-3008.

2. Krishnan, R., et al., Structure of thrombin complexed with selective non-electrophilic inhibitors having cyclohexyl moieties at P1. Acta Crystallogr D Biol Crystallogr, 2000. 56(Pt 3): p. 294-303.

3. Baum, B., et al., Think twice: understanding the high potency of bis(phenyl)methane inhibitors of thrombin. $\mathrm{J} \mathrm{Mol}$ Biol, 2009. 391(3): p. 552-64.

4. Tucker, T.J., et al., Design and synthesis of a series of potent and orally bioavailable noncovalent thrombin inhibitors that utilize nonbasic groups in the P1 position. J Med Chem, 1998. 41(17): p. 3210-9.

5. $\quad$ Nantermet, P.G., et al., Design and synthesis of potent and selective macrocyclic thrombin inhibitors. Bioorg Med Chem Lett, 2003. 13(16): p. 2781-4.

6. Malley, M.F., et al., Crystallographic determination of the structures of human alpha-thrombin complexed with BMS-186282 and BMS-189090. Protein Sci, 1996. 5(2): p. 221-8.

7. Brandstetter, H., et al., Refined 2.3 A X-ray crystal structure of bovine thrombin complexes formed with the benzamidine and arginine-based thrombin inhibitors NAPAP, 4-TAPAP and MQPA. A starting point for improving antithrombotics. J Mol Biol, 1992. 226(4): p. 1085-99.

8. Senger, S., et al., Sulfonamide-related conformational effects and their importance in structure-based design. Bioorg Med Chem Lett, 2007. 17(10): p. 2931-4.

9. Weir, M.P., et al., Novel natural product 5,5-trans-lactone inhibitors of human alpha-thrombin: mechanism of action and structural studies. Biochemistry, 1998. 37(19): p. 6645-57.

10. Biela, A., et al., Impact of ligand and protein desolvation on ligand binding to the S1 pocket of thrombin. $\mathrm{J}$ Mol Biol, 2012. 418(5): p. 350-66.

11. Gerlach, C., et al., KNOBLE: a knowledge-based approach for the design and synthesis of readily accessible small-molecule chemical probes to test protein binding. Angew Chem Int Ed Engl, 2007. 46(47): p. 9105-9.

12. Ye, B., et al., Thiophene-anthranilamides as highly potent and orally available factor Xa inhibitors. J Med Chem, 2007. 50(13): p. 2967-80.

13. Adler, M., et al., Crystal structures of two potent nonamidine inhibitors bound to factor Xa. Biochemistry, 2002. 41(52): p. 15514-23.

14. Qiao, J.X., et al., Achieving structural diversity using the perpendicular conformation of alpha-substituted phenylcyclopropanes to mimic the bioactive conformation of ortho-substituted biphenyl P4 moieties: discovery of novel, highly potent inhibitors of Factor Xa. Bioorg Med Chem Lett, 2008. 18(14): p. 4118-23.

15. Pinto, D.J., et al., 1-[3-Aminobenzisoxazol-5'-yl]-3-trifluoromethyl-6-[2'-(3-(R)-hydroxy-N-pyrrolidin yl)methyl[1,1']-biphen-4-yl]-1,4,5,6-tetrahydropyrazolo-[3,4-c]-pyridin-7-one (BMS-740808) a highly potent, selective, efficacious, and orally bioavailable inhibitor of blood coagulation factor Xa. Bioorg Med Chem Lett, 2006. 16(15): p. 4141-7.

16. Nazare, M., et al., Probing the subpockets of factor Xa reveals two binding modes for inhibitors based on a 2 carboxyindole scaffold: a study combining structure-activity relationship and X-ray crystallography. J Med Chem, 2005. 48(14): p. 4511-25.

17. Pinto, D.J., et al., Discovery of 1-(4-methoxyphenyl)-7-oxo-6-(4-(2-oxopiperidin-1-yl)phenyl)-4,5,6,7-tetrahydro$1 H$-pyrazolo[3,4-c]pyridine-3-carboxamide (apixaban, BMS-562247), a highly potent, selective, efficacious, and orally bioavailable inhibitor of blood coagulation factor Xa. J Med Chem, 2007. 50(22): p. 5339-56.

18. Adler, M., et al., Preparation, characterization, and the crystal structure of the inhibitor ZK-807834 (CI-1031) complexed with factor Xa. Biochemistry, 2000. 39(41): p. 12534-42.

19. Pinto, D.J., et al., Discovery of potent, efficacious, and orally bioavailable inhibitors of blood coagulation factor Xa with neutral P1 moieties. Bioorg Med Chem Lett, 2006. 16(21): p. 5584-9.

20. Quan, M.L., et al., Discovery of 1-(3'-aminobenzisoxazol-5'-yl)-3-trifluoromethyl-N-[2-fluoro-4- [(2'dimethylaminomethyl)imidazol-1-yl]phenyl]-1H-pyrazole-5-carboxyamide hydrochloride (razaxaban), a highly potent, selective, and orally bioavailable factor Xa inhibitor. J Med Chem, 2005. 48(6): p. 1729-44.

21. Roehrig, S., et al., Discovery of the novel antithrombotic agent 5-chloro-N-(\{(5S)-2-oxo-3- [4-(3-oxomorpholin-4yl)phenyl]-1,3-oxazolidin-5-yl\}methyl)thiophene- 2-carboxamide (BAY 59-7939): an oral, direct factor Xa inhibitor. J Med Chem, 2005. 48(19): p. 5900-8.

22. Qiao, J.X., et al., SAR and X-ray structures of enantiopure 1,2-cis-(1R,2S)-cyclopentyldiamine and cyclohexyldiamine derivatives as inhibitors of coagulation Factor Xa. Bioorg Med Chem Lett, 2007. 17(16): $\mathrm{p}$. 4419-27.

23. Young, R.J., et al., Structure- and property-based design of factor Xa inhibitors: pyrrolidin-2-ones with acyclic alanyl amides as P4 motifs. Bioorg Med Chem Lett, 2006. 16(23): p. 5953-7.

24. Chan, C., et al., Factor Xa inhibitors: S1 binding interactions of a series of $N-\{(3 S)-1-[(1 S)-1-m e t h y l-2-m o r p h o l i n-$ 4-yl-2-oxoethyl]-2-oxopyrrolidin-3-yl\}sulfon amides. J Med Chem, 2007. 50(7): p. 1546-57.

25. Senger, S., et al., Arylsulfonamides: a study of the relationship between activity and conformational preferences for a series of factor Xa inhibitors. Bioorg Med Chem Lett, 2006. 16(22): p. 5731-5.

26. Brandstetter, H., et al., X-ray structure of active site-inhibited clotting factor Xa. Implications for drug design and substrate recognition. J Biol Chem, 1996. 271(47): p. 29988-92.

27. Young, R.J., et al., Selective and dual action orally active inhibitors of thrombin and factor Xa. Bioorg Med Chem Lett, 2007. 17(10): p. 2927-30.

28. Hosur, M.V., et al., Influence of stereochemistry on activity and binding modes for C2 symmetry-based diol inhibitors of HIV-1 protease. Journal of the American Chemical Society, 1994. 116(3): p. 847-855. 
29. Andersson, H.O., et al., Optimization of P1-P3 groups in symmetric and asymmetric HIV-1 protease inhibitors. Eur J Biochem, 2003. 270(8): p. 1746-58.

30. Munshi, S., et al., An alternate binding site for the P1-P3 group of a class of potent HIV-1 protease inhibitors as a result of concerted structural change in the 80s loop of the protease. Acta Crystallogr D Biol Crystallogr, 2000. 56(Pt 4): p. 381-8.

31. Munshi, S., et al., Rapid X-ray diffraction analysis of HIV-1 protease-inhibitor complexes: inhibitor exchange in single crystals of the bound enzyme. Acta Crystallogr D Biol Crystallogr, 1998. 54(Pt 5): p. 1053-60.

32. Ekegren, J.K., et al., Microwave-accelerated synthesis of P1'-extended HIV-1 protease inhibitors encompassing a tertiary alcohol in the transition-state mimicking scaffold. J Med Chem, 2006. 49(5): p. 1828-32.

33. $\mathrm{Wu}, \mathrm{X}$., et al., Two-carbon-elongated HIV-1 protease inhibitors with a tertiary-alcohol-containing transition-state mimic. J Med Chem, 2008. 51(4): p. 1053-7.

34. Schaal, W., et al., Synthesis and comparative molecular field analysis (CoMFA) of symmetric and nonsymmetric cyclic sulfamide HIV-1 protease inhibitors. J Med Chem, 2001. 44(2): p. 155-69.

35. Ekegren, J.K., et al., A new class of HIV-1 protease inhibitors containing a tertiary alcohol in the transition-state mimicking scaffold. J Med Chem, 2005. 48(25): p. 8098-102.

36. Abdel-Meguid, S.S., et al., An orally bioavailable HIV-1 protease inhibitor containing an imidazole-derived peptide bond replacement: crystallographic and pharmacokinetic analysis. Biochemistry, 1994. 33(39): p. 116717.

37. Thaisrivongs, S., et al., Structure-based design of novel HIV protease inhibitors: carboxamide-containing 4hydroxycoumarins and 4-hydroxy-2-pyrones as potent nonpeptidic inhibitors. J Med Chem, 1995. 38(18): p. 362437.

38. Blum, A., et al., Structure-guided design of C2-symmetric HIV-1 protease inhibitors based on a pyrrolidine scaffold. J Med Chem, 2008. 51(7): p. 2078-87.

39. Hoog, S.S., et al., A check on rational drug design: crystal structure of a complex of human immunodeficiency virus type 1 protease with a novel gamma-turn mimetic inhibitor. J Med Chem, 1995. 38(17): p. 3246-52.

40. Blum, A., et al., Achiral oligoamines as versatile tool for the development of aspartic protease inhibitors. Bioorg Med Chem, 2008. 16(18): p. 8574-86.

41. Lee, T., et al., Discovery of Potent Myeloid Cell Leukemia-1 (Mcl-1) Inhibitors That Demonstrate in Vivo Activity in Mouse Xenograft Models of Human Cancer. J Med Chem, 2019. 62(8): p. 3971-3988.

42. Caenepeel, S., et al., AMG 176, a Selective MCL1 Inhibitor, Is Effective in Hematologic Cancer Models Alone and in Combination with Established Therapies. Cancer Discov, 2018. 8(12): p. 1582-1597.

43. Rescourio, G., et al., Discovery and in Vivo Evaluation of Macrocyclic Mcl-1 Inhibitors Featuring an alphaHydroxy Phenylacetic Acid Pharmacophore or Bioisostere. J Med Chem, 2019. 62(22): p. 10258-10271.

44. $\quad$ Pelz, N.F., et al., Discovery of 2-Indole-acylsulfonamide Myeloid Cell Leukemia 1 (Mcl-1) Inhibitors Using Fragment-Based Methods. J Med Chem, 2016. 59(5): p. 2054-66.

45. Shaw, S., et al., Optimization of Potent and Selective Tricyclic Indole Diazepinone Myeloid Cell Leukemia-1 Inhibitors Using Structure-Based Design. J Med Chem, 2018. 61(6): p. 2410-2421.

46. Bruncko, M., et al., Structure-guided design of a series of MCL-1 inhibitors with high affinity and selectivity. J Med Chem, 2015. 58(5): p. 2180-94.

47. Lee, T., et al., Discovery and biological characterization of potent myeloid cell leukemia-1 inhibitors. FEBS Lett, 2017. 591(1): p. 240-251.

48. Szlavik, Z., et al., Structure-Guided Discovery of a Selective Mcl-1 Inhibitor with Cellular Activity. J Med Chem, 2019. 62(15): p. 6913-6924.

49. Friberg, A., et al., Discovery of potent myeloid cell leukemia 1 (Mcl-1) inhibitors using fragment-based methods and structure-based design. J Med Chem, 2013. 56(1): p. 15-30.

50. Burke, J.P., et al., Discovery of tricyclic indoles that potently inhibit Mcl-1 using fragment-based methods and structure-based design. J Med Chem, 2015. 58(9): p. 3794-805.

51. Chu, X.J., et al., Discovery of [4-Amino-2-(1-methanesulfonylpiperidin-4-ylamino)pyrimidin-5-yl](2,3-difluoro-6methoxyphenyl)methanone (R547), a potent and selective cyclin-dependent kinase inhibitor with significant in vivo antitumor activity. J Med Chem, 2006. 49(22): p. 6549-60.

52. Hole, A.J., et al., Comparative structural and functional studies of 4-(thiazol-5-yl)-2-(phenylamino)pyrimidine-5carbonitrile CDK9 inhibitors suggest the basis for isotype selectivity. J Med Chem, 2013. 56(3): p. 660-70.

53. Moshinsky, D.J., et al., SU9516: biochemical analysis of cdk inhibition and crystal structure in complex with cdk2. Biochem Biophys Res Commun, 2003. 310(3): p. 1026-31.

54. Misra, R.N., et al., N-(cycloalkylamino)acyl-2-aminothiazole inhibitors of cyclin-dependent kinase 2. N-[5-[[[5(1,1-dimethylethyl)-2-oxazolyl]methyl]thiol-2-thiazolyl]-4- piperidinecarboxamide (BMS-387032), a highly efficacious and selective antitumor agent. J Med Chem, 2004. 47(7): p. 1719-28.

55. Wang, S., et al., 2-Anilino-4-(thiazol-5-yl)pyrimidine CDK inhibitors: synthesis, SAR analysis, X-ray crystallography, and biological activity. J Med Chem, 2004. 47(7): p. 1662-75.

56. Berg, S., et al., Discovery of novel potent and highly selective glycogen synthase kinase-3beta (GSK3beta) inhibitors for Alzheimer's disease: design, synthesis, and characterization of pyrazines. J Med Chem, 2012. 55(21): p. 9107-19.

57. Wu, S.Y., et al., Discovery of a novel family of CDK inhibitors with the program LIDAEUS: structural basis for ligand-induced disordering of the activation loop. Structure, 2003. 11(4): p. 399-410.

58. Hamdouchi, C., et al., The discovery of a new structural class of cyclin-dependent kinase inhibitors, aminoimidazo[1,2-a]pyridines. Mol Cancer Ther, 2004. 3(1): p. 1-9.

59. Echalier, A., et al., An integrated chemical biology approach provides insight into Cdk2 functional redundancy and inhibitor sensitivity. Chem Biol, 2012. 19(8): p. 1028-40.

60. Shao, H., et al., Substituted 4-(thiazol-5-yl)-2-(phenylamino)pyrimidines are highly active CDK9 inhibitors: synthesis, X-ray crystal structures, structure-activity relationship, and anticancer activities. J Med Chem, 2013. 56(3): p. 640-59. 
61. Arris, C.E., et al., Identification of novel purine and pyrimidine cyclin-dependent kinase inhibitors with distinct molecular interactions and tumor cell growth inhibition profiles. J Med Chem, 2000. 43(15): p. 2797-804.

62. Clare, P.M., et al., The cyclin-dependent kinases cdk2 and cdk5 act by a random, anticooperative kinetic mechanism. J Biol Chem, 2001. 276(51): p. 48292-9.

63. Sayle, K.L., et al., Structure-based design of 2-arylamino-4-cyclohexylmethyl-5-nitroso-6-aminopyrimidine inhibitors of cyclin-dependent kinases 1 and 2. Bioorg Med Chem Lett, 2003. 13(18): p. 3079-82.

64. Beattie, J.F., et al., Cyclin-dependent kinase 4 inhibitors as a treatment for cancer. Part 1: identification and optimisation of substituted 4,6-bis anilino pyrimidines. Bioorg Med Chem Lett, 2003. 13(18): p. 2955-60. 